\title{
Prediction and Assessment of the Surface-based Aeroacoustics of Vertical-axis Wind Turbines
}

by

Robert Williams, B.Eng.

\author{
A thesis submitted to the \\ Faculty of Graduate and Postdoctoral Affairs \\ in partial fulfillment of the requirements for the degree of
}

Master of Applied Science

Ottawa-Carleton Institute for Mechanical and Aerospace Engineering

Department of Mechanical and Aerospace Engineering

Carleton University

Ottawa, Ontario

December, 2014

(C) Copyright

Robert Williams, 2014 
The undersigned hereby recommends to the

Faculty of Graduate and Postdoctoral Affairs acceptance of the thesis

\title{
Prediction and Assessment of the Surface-based Aeroacoustics of Vertical-axis Wind Turbines
}

\author{
submitted by Robert Williams, B.Eng. \\ in partial fulfillment of the requirements for the degree of
}

Master of Applied Science

Professor Joana Rocha, Thesis Co-supervisor

Professor Edgar Matida, Thesis Co-supervisor

Professor Fred Nitzsche, Thesis Co-supervisor

Professor Metin Yaras, Chair, Department of Mechanical and Aerospace Engineering

Ottawa-Carleton Institute for Electrical and Computer Engineering Department of Mechanical and Aerospace Engineering Carleton University

December, 2014 


\section{Abstract}

Aerodynamic and aeroacoustic prediction tools are developed for rigid rotor blades in unsteady motion to provide insight into the aeroacoustics of vertical-axis wind turbine rotor blades. The aerodynamic component uses a subsonic unsteady inviscid panel method over the surface of the rotor blades to predict the unsteady pressure distribution over the surface, with vortex particles being shed from the blades to represent their freely-convecting wake. Using the velocity and pressure distributions over the rotor blades, the aeroacoustic component employs a non-penetrable version of Formulation $1 \mathrm{C}$ of the Ffowcs Williams-Hawkings equation to predict the noise from surface-based acoustic sources called thickness and loading noise. The prediction tools are compared to accepted results for fundamental test cases and vertical-axis wind turbines before being used to investigate the aerodynamics and acoustic noise of vertical-axis wind turbine rotors. Investigations into the effects of the blade geometry, the geometric scale of the rotor, the number of rotor blades, and the tip speed ratio of the rotor on the acoustic field are presented. The acoustic results are expected to under-predict the total acoustic noise of vertical-axis wind turbines. 
In memory of Christopher H. Williams 


\section{Acknowledgments}

Throughout my graduate research, I have been fortunate enough to have worked under the supervision of Professor Joana Rocha, Professor Edgar Matida, and Professor Fred Nitzsche. Their consistent guidance, wisdom, and all-around support have been very helpful throughout my time with them, for which I am very grateful.

In times of trouble and when research tasks became overly daunting, several people have come to my aid to help guide me through. My thanks go out to Amin Fereidooni for patiently introducing me to the concept of vertical-axis wind turbines and yet another new programming language to learn, Daniel Opoku for graciously attempting to explain concepts of panel methods and vortex particle methods, and Sean McTavish for providing me with a wealth of knowledge about the present state of wind turbine research.

My work has been generously supported by the Ontario Graduate Scholarship program, the Natural Sciences and Engineering Research Council of Canada, which have provided a welcome sense of piece-of-mind throughout my studies.

Last but not least, I would like to thank my family for their endless support, and in particular my mother for her editorial contributions and occasional left-overs to keep myself well-fed throughout this time. 


\section{Table of Contents}

Abstract $\quad$ iii

Acknowledgments $\quad$ v

Table of Contents vi vi

List of Tables $\quad$ ix

List of Figures $\quad$ x

List of Symbols $\quad$ xiv

List of Acronyms xix

1 Introduction 1

1.1 Background ........................... 1

1.2 Motivation ........................... 5

1.3 Fundamentals of VAWTs . . . . . . . . . . . . . . . . 5

1.3.1 Theory of VAWT operations . . . . . . . . . . . . 6

1.3.2 VAWT parameters .................. 9

1.4 State of the art . . . . . . . . . . . . . . . 11

1.4.1 Aeroacoustics ................... 11

1.4.2 Aerodynamics . . . . . . . . . . . . . 15 
1.5 Structure of the thesis $\ldots \ldots \ldots \ldots \ldots$

2 Aerodynamic Analysis $\quad 19$

2.1 Aerodynamic methods . . . . . . . . . . . . . . . . . . . 19

2.1.1 Singularity elements . . . . . . . . . . . . . 20

2.1 .2 Geometric representation . . . . . . . . . . . . . . . 26

2.1 .3 Wake representation . . . . . . . . . . . . . . 28

2.1.4 Solving the panel method . . . . . . . . . . . . . . 34

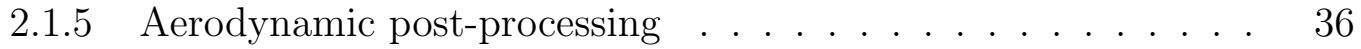

2.2 Aerodynamic component implementation . . . . . . . . . . . . 37

2.3 Aerodynamic model verification and validation . . . . . . . . . 39

3 Aeroacoustic Analysis $\quad 43$

3.1 Aeroacoustic concepts . . . . . . . . . . . . . . . . . 43

3.2 Aeroacoustic methods . . . . . . . . . . . . . . . . . 44

3.2 .1 Advanced time approach . . . . . . . . . . . . . . . 45

3.2 .2 Formulation $1 \mathrm{C} \ldots \ldots \ldots \ldots \ldots \ldots$

3.2.3 Aeroacoustic component implementation . . . . . . . . . 50

3.3 Aeroacoustic model verification . . . . . . . . . . . . . . . . 53

4 Results and Discussions $\quad 57$

4.1 Convergence Analyses $\ldots \ldots \ldots \ldots \ldots$

4.1.1 Spatial Convergence . . . . . . . . . . . . . 58

4.1 .2 Temporal Convergence . . . . . . . . . . . . . . . . . . 59

4.2 Verification and Validation for VAWTs . . . . . . . . . . . 63

4.2.1 Aerodynamic Verification . . . . . . . . . . . . 63

4.2 .2 Aeroacoustic Validation . . . . . . . . . . . . . 66 
4.3 Parametric Investigations . . . . . . . . . . . . . . . . . . . 68

4.3.1 Effects due to Different Rotor Geometries . . . . . . . . . 68

4.3.2 Effects due to Varying Rotor Scale _ . . . . . . . . . . 70

4.3.3 Effects due to the Number of Rotor Blades . . . . . . . . . . 70

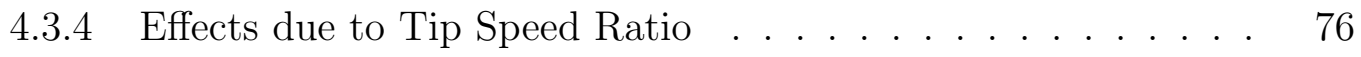

5 Summary, Conclusions, and Recommendations 78

5.1 Summary and Contributions . . . . . . . . . . . . 78

5.2 Conclusions . . . . . . . . . . . . . . . . . . . . . . . . . 79

5.3 Recommendations . . . . . . . . . . . . . . . . . . . 80

$\begin{array}{lc}\text { List of References } & 83\end{array}$

Appendix A Equations for Aerodynamic Singularity Elements $\quad 89$

A.1 Three-dimensional quadrilateral panels . . . . . . . . . . . 90

A.1.1 3D uniform-strength source panels . . . . . . . . . 91

A.1.2 3D uniform-strength doublet panels . . . . . . . . . . . 92

A.2 Three-dimensional vortex particles . . . . . . . . . . . . 93

A.3 Transformation from doublet panels to vortex filaments and vortex

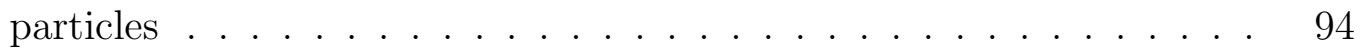

\section{$\begin{array}{ll}\text { Appendix B Investigating Aeroacoustic Formulations } & 99\end{array}$}

B.1 Derivation of Formulation 1C for non-penetrable surfaces . . . . . 99 


\section{List of Tables}

4.1 Parameters of the Sandia $17-m$ VAWT, $[56] \ldots \ldots \ldots \ldots$

4.2 Parameters of the aerodynamic verification turbine . . . . . . . 64

4.3 Overview of the parameters being investigated . . . . . . . 68

4.4 Constants throughout all investigations . . . . . . . . . . . 69 


\section{List of Figures}

1.1 Photographs of (a) the 64-metre diameter, 3.6MW Eole VAWT [8], and (b) a 5-metre diameter, 50kW straight-bladed VAWT [9] . . . .

1.2 Top view of a horizontal-section of a VAWT with rotor blade positions $\theta_{\mathrm{T}}=0^{\circ}, 90^{\circ}, 180^{\circ}$, and $270^{\circ}$ shown . . . . . . . . . 7

1.3 Plot of the geometric angle of attack of a section of VAWT blade over the rotation with various local speed ratios . . . . . . . . . . 8

2.1 Illustrations of a point source, point doublet, and point vortex in two-

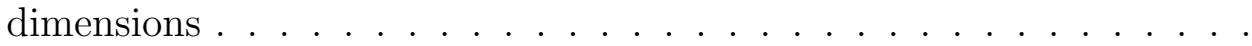

2.2 Streamlines and arrows representing velocity fields around uniformstrength quadrilateral panels . . . . . . . . . . . . . .

2.3 VP perturbation velocity as a function of radial distance from the VP, where $r=|\mathbf{r}|,|\boldsymbol{\xi}|=1$, and $\boldsymbol{\xi} \perp \mathbf{r} \ldots \ldots \ldots 25$

2.4 Streamlines and arrows representing the velocity field around a VP with a non-zero smoothing radius . . . . . . . . . . . . .

2.5 Illustration of a geometric discretization of a Troposkien rotor with a NACA 0012 airfoil . . . . . . . . . . . . . . . . . 27

2.6 Illustration of a single near-wake panel row for a wing with $N_{h}=3$. 
2.7 Curves illustrating the positioning of the trailing-edge (TE) of the near wake using: (a) free-stream velocity, (b) bisector angle of TE, and (c) kinematic rotation . . . . . . . . . . . . . . . 30

2.8 Coefficient of pressure distributions for a NACA 0012 airfoil at $\alpha=5^{\circ}$ and $\alpha=10^{\circ} \ldots \ldots \ldots \ldots \ldots \ldots \ldots \ldots \ldots \ldots \ldots$

2.9 Span-wise distributions of lift coefficient along a wing in steady flow . 41

2.10 Unsteady lift coefficient for a pitching oscillation of a NACA 0012 airfoil 42

2.11 VPs shed from an oscillating wing . . . . . . . . . . . . 42

3.1 Illustration of the relationship between source and observer time frames for two moving acoustic sources (shown as red and blue) at separate

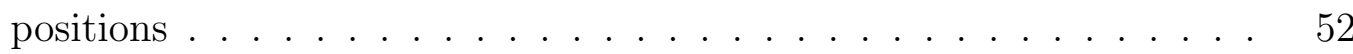

3.2 Monopole perturbation pressure radiation pattern at an observer dis-

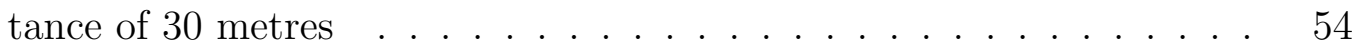

3.3 Dipole perturbation pressure radiation pattern at an observer distance of 30 metres for a dipole oriented in the $90^{\circ}$ direction . . . . . . 55

3.4 A schematic of the rotating monopole and observer positions and the observed perturbation pressure histories $\ldots \ldots \ldots \ldots$

4.1 Profile of the Sandia 17 -m rotor, based on $[57] \ldots \ldots \ldots$

4.2 Convergence of rotor span-wise discretization using steady-state nondimensional forces $F_{T}^{+}$and $F_{N}^{+}$at the equator $\ldots \ldots \ldots 60$

4.3 Convergence of rotor airfoil discretization using steady-state nondimensional forces $F_{T}^{+}$and $F_{N}^{+}$at the equator . . . . . . . . 61

4.4 Convergence of the root mean square (RMS) of acoustic pressure for decreasing increment of azimuth angle . . . . . . . . . . . . 62

4.5 Acoustic pressure PSD for decreasing azimuth increment size . . . . . 62

4.6 Profile of the aerodynamic verification rotor $\ldots \ldots \ldots \ldots$ 
4.7 Comparison of equator forces predicted by the present method and the

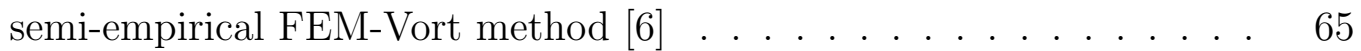

4.8 Comparison of PSD of the Sandia 17-m VAWT between experimental and present method results . . . . . . . . . . . . . .

4.9 Illustration of the basic Troposkein turbine considered in the present

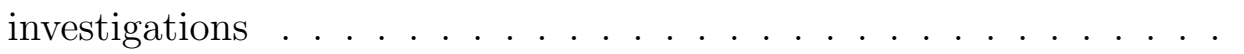

4.10 Non-dimensional tangential force $F_{T}^{+}$over the height a Troposkein blade (top) and a straight blade (bottom) throughout one rotation . . 71

4.11 Top (top) and side (bottom) views of a visualization of the Troposkein rotor and its VP wake . . . . . . . . . . . . . . 72

4.12 Top (top) and side (bottom) views of a visualization of a straight-blade rotor and its VP wake . . . . . . . . . . . . . . . . . 72

4.13 SPL directivity plots for Troposkein and straight-blade rotors at an observer distance of $3 d_{\mathrm{T}} \ldots \ldots \ldots$. . . . . . . . . . . . . . . 73

4.14 Comparison of the PSD of a Troposkein geometry and a straight-bladed geometry for an observer $3 d_{\mathrm{T}}$ upwind from the turbine axis . . . . . . 74

4.15 SPL of various geometric scales of Troposkein VAWTs for observers at varying distances upwind from the turbine axis . . . . . . . . . . 74

4.16 SPL directivity plots for Troposkein rotors with various numbers of blades ............................ 75

4.17 SPL directivity plots for a Troposkein rotors operating at various TSRs 76

A.1 Near-wake panels and vortex filaments from previous time step after being convected . . . . . . . . . . . . . . . 95

A.2 Convected near-wake after conversion to vortex filament representation 95

A.3 Convected near-wake after conversion to vortex filament representation and one panel converted to a vortex particle . . . . . . . . . . . 96 
A.4 Convected near-wake after conversion to vortex filament representation and two panels converted to vortex particles . . . . . . . . . . 96

A.5 Convected near-wake after conversion to vortex filament representation and three panels converted to vortex particles . . . . . . . . . . 96

A.6 The new near-wake doublet panels and far-wake vortex filaments and

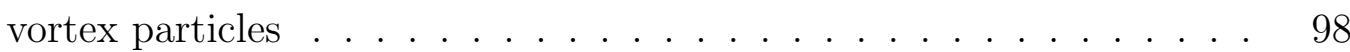




\section{List of Symbols}

$A_{i j} \quad$ Influence coefficient of surface doublet panel $j$ on control point of surface panel $i$, including near-wake panel effects

$A_{\text {swept }} \quad$ Swept area of the turbine rotor, $\left[\mathrm{m}^{2}\right]$

R Aspect ratio

$B_{i j} \quad$ Influence coefficient of surface source panel $j$ on control point of surface panel $i$

$B P F \quad$ Blade passage frequency, $[\mathrm{Hz}]$

$c_{0} \quad$ Speed of sound in the undisturbed medium, $[\mathrm{m} / \mathrm{s}]$

$C_{i j} \quad$ Influence coefficient of surface doublet panel $j$ on control point of surface panel $i$

$C_{i w} \quad$ Influence coefficient of near-wake doublet panel $w$ on control point of surface panel $i$

$C_{p} \quad$ Coefficient of pressure, dimensionless gauge pressure on the surface

$c_{\mathrm{T}} \quad$ Chord length of the turbine rotor blades, $[\mathrm{m}]$ 
D Drag vector, $[\mathrm{N}]$

$d_{\mathrm{T}} \quad$ Diameter of the turbine rotor, $[\mathrm{m}]$

$f \quad$ Equation of the body surface

$\mathbf{F}_{\mathbf{N}} \quad$ Force in the rotor's normal direction, $[\mathrm{N}]$

$F_{N}^{+} \quad$ Non-dimensional force in the rotor's normal direction

$\mathbf{F}_{\mathbf{T}} \quad$ Force in the rotor's tangential direction, $[\mathrm{N}]$

$F_{T}^{+} \quad$ Non-dimensional force in the rotor's tangential direction

$\left(\frac{h}{d}\right)_{\mathrm{T}} \quad$ Height-to-diameter ratio of the turbine rotor blades

$h_{\mathrm{T}} \quad$ Height of the turbine rotor, [m]

L Lift vector, $[\mathrm{N}]$

$L_{n i} \quad$ Vector quantity representing acoustic loading effects, $[\mathrm{Pa}]$

$L_{i j} \quad$ Tensor quantity representing acoustic loading effects, $[\mathrm{Pa}]$

$\hat{\mathbf{n}} \quad$ Unit normal vector to the surface in the outwards direction

$N \quad$ Number of surface panels

$N_{a} \quad$ Number of line segments in a discretized airfoil profile

$N_{b} \quad$ Number of rotor blades in the turbine rotor

$N_{h} \quad$ Number of span-wise sections of a discretized rotor blade

$N_{\mathrm{VP}} \quad$ Number of existing vortex particles 


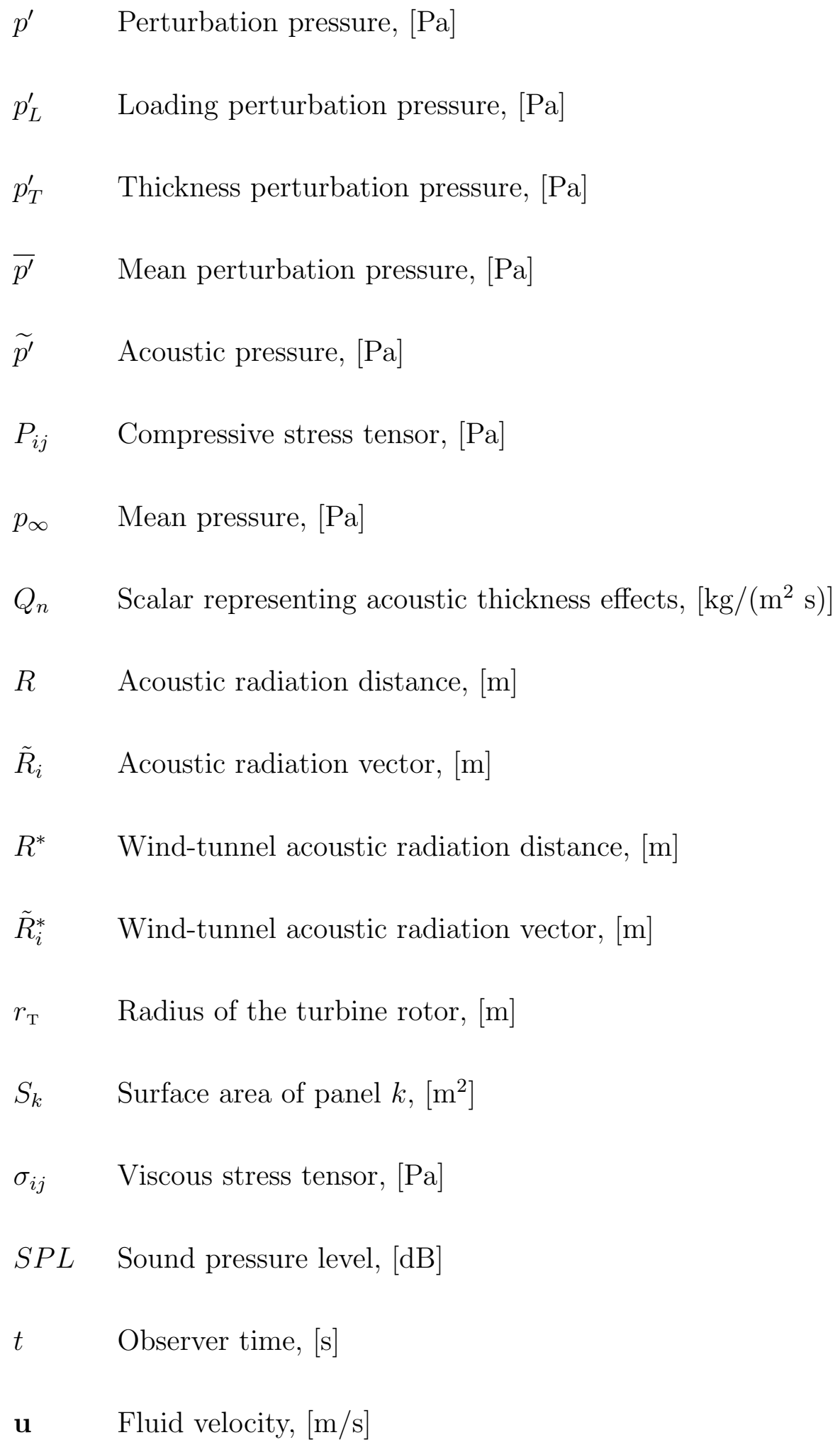


$\mathbf{u}^{\prime} \quad$ Flow perturbation velocity vector, $[\mathrm{m} / \mathrm{s}]$

$\mathbf{U}_{\infty} \quad$ Free-stream velocity vector, $[\mathrm{m} / \mathrm{s}]$

V Kinematic velocity, $[\mathrm{m} / \mathrm{s}]$

$\mathbf{v} \quad$ Kinematic velocity the surface, $[\mathrm{m} / \mathrm{s}]$

$\mathbf{x} \quad$ Observer position vector in the inertial reference frame, $[\mathrm{m}]$

$\boldsymbol{\xi} \quad$ Vortex particle intensity, $\left[\mathrm{m}^{3} / \mathrm{s}\right]$

y Source position vector in the inertial reference frame, [m]

$\mathbf{Z} \quad$ Vortex particle position, $[\mathrm{m}]$

$\alpha \quad$ Angle of attack, $[\mathrm{rad}]$ or $\left[^{\circ}\right]$

$\beta \quad$ Prandtl-Glauert factor

$\delta_{i j} \quad$ Kronecker delta

$\epsilon \quad$ Vortex particle smoothing radius, [m]

$\lambda_{\mathrm{T}} \quad$ Turbine rotor tip-speed ratio

$\mu \quad$ Doublet panel strength, $[\mathrm{m} / \mathrm{s}]$

$\boldsymbol{\omega} \quad$ Vorticity vector, $\left[\mathrm{m}^{3} / \mathrm{s}\right]$

$\Omega_{\mathrm{T}} \quad$ Turbine rotational velocity, $[\mathrm{rad} / \mathrm{s}]$

$\Phi \quad$ Velocity potential, $[\mathrm{m} / \mathrm{s}]$ 
$\rho \quad$ Fluid density, $\left[\mathrm{kg} / \mathrm{m}^{3}\right]$

$\rho_{0} \quad$ Fluid density in the undisturbed medium, $\left[\mathrm{kg} / \mathrm{m}^{3}\right]$

$\sigma \quad$ Source panel strength, $[\mathrm{m} / \mathrm{s}]$

$\sigma_{\mathrm{T}} \quad$ Solidity of the turbine rotor blades

$\tau \quad$ Source time, $[\mathrm{s}]$

$\theta_{\mathrm{T}} \quad$ Turbine rotor azimuth angle, measured counter-clockwise about turbine axis from downwind direction when viewed from the top, [rad] or $\left[^{\circ}\right]$

$\zeta_{\epsilon} \quad$ Vortex particle smoothing function 


\title{
List of Acronyms
}

\author{
BC boundary condition \\ BPF blade passage frequency \\ CFD computational fluid dynamics \\ FW-H Ffowcs Williams-Hawkings \\ HAWT horizontal-axis wind turbine \\ PSD power spectral density \\ RMS root mean square \\ SPL sound pressure level \\ TE trailing-edge \\ TSR tip speed ratio \\ VAWT vertical-axis wind turbine \\ VP vortex particle
}




\section{Chapter 1}

\section{Introduction}

As modern societies seek to decrease their dependence on fossil-fuel-based energy sources and increase their use of sustainable resources for electrical energy generation, wind turbines are often being turned to as a means to produce electricity. Commercial wind turbines are growing larger, small wind turbines are being used in urban environments, and the design of wind turbines is being adapted to suit these applications [1-3]. The Darrieus vertical-axis wind turbine (VAWT) is one classification of wind turbine which is regaining academic and commercial interest, but has not yet been researched as extensively as the more common horizontal-axis wind turbine (HAWT) and its aeroacoustic noise characteristics $[4,5]$.

\subsection{Background}

Wind turbines are machines which extract energy from the horizontal motion of atmospheric air called "wind". Often referred to as a renewable source of energy, wind varies with space and time in direction and intensity. Wind turbines are windpowered generators which convert the kinetic energy of the wind to mechanical or electrical energy [2]. 
There are two main categories of wind turbines: HAWTs and VAWTs [2]. To capture the kinetic energy of the wind, HAWTs use propeller-type rotors with their rotational axis horizontal and parallel to the direction of the wind. VAWTs differ from HAWTs by using rotors with a rotational axis that is vertical and therefore perpendicular to the direction of the wind. Both categories require similar mechanical components, including a rotor which extracts the energy from the wind, a tower which supports the rotor, and a gearbox which changes the rotational speed of the rotor shaft $[2]$.

VAWT rotors can be further categorized into those which are driven by aerodynamic drag, and those which are driven by aerodynamic lift. Due to larger limitations in its maximum power coefficient and the large quantity of material required, dragdriven rotors such as Savonius rotors are best suited for small power requirements [2]. Lift-driven VAWT rotors, often referred to as Darrieus rotors or Darrieus wind turbines, use airfoiled blades to increase the ratio of lift-to-drag which permits higher efficiencies than Savonius rotors [1,2]. For an explanation of how Darrieus VAWTs function, refer to Sec. 1.3. Unless explicitly stated otherwise, the use of the term VAWT in this thesis shall refer to a VAWT of the Darrieus type.

A wide variety of VAWT rotor designs exist, with the curved Troposkien-shaped blades and the Giromill's straight blades being most common. VAWT rotors with Troposkien geometries, as in Fig. 1.1a, have rotor blades which are curved to follow the shape which a perfectly flexible cable with two fixed ends would take if it were spun about an axis passing through its ends [2,6]. Giromill VAWT rotors, on the other hand, have a number of straight blades oriented parallel to the axis of rotation, as seen in Fig. 1.1b. The Troposkien shape is derived on the basis of a uniform density blade's centrifugal forces contributing solely to tension in the blade [2], and therefore minimizes bending stresses in the blades [7]. Large VAWTs typically have 


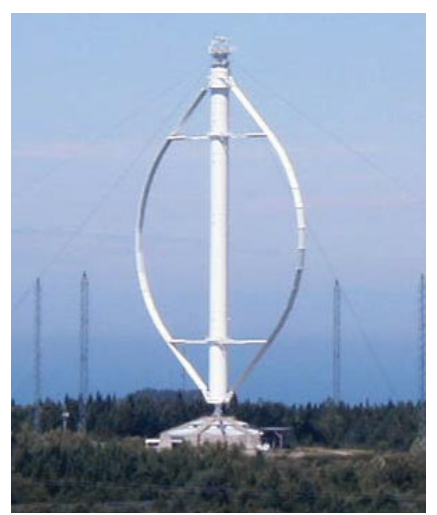

(a)

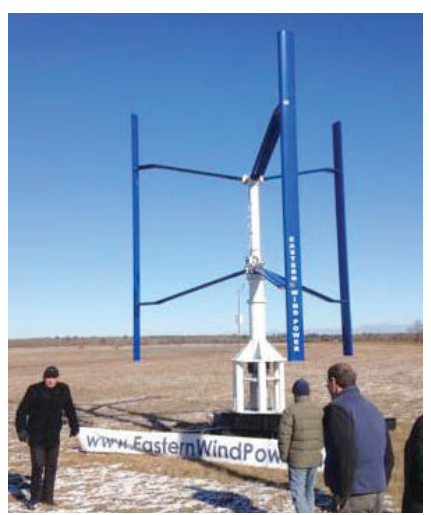

(b)

Figure 1.1: Photographs of (a) the 64-metre diameter, 3.6MW Eole VAWT [8], and (b) a 5-metre diameter, 50kW straight-bladed VAWT [9]

Troposkien- or nearly Troposkien-shaped blades as the complexity of their geometry is warranted by the need to minimize bending loads.

The largest VAWT known by the author to exist is the Troposkien-shaped Lavalin Eole Research Turbine, as seen in Fig. 1.1a, which has a diameter of 64 metres and was operated commercially between 1988 and 1993 in Cap Chat, Quebec, Canada, with a design power output of $3.6 \mathrm{MW}[2,10]$. In fact, Rangi and South from the National Research Council of Canada are credited with rediscovering the Darrieus turbine circa 1966, which led to commercialization of the design through Sandia National Laboratories [11].

While HAWTs are the more common design, VAWTs are more advantageous than HAWTs in their relative insensitivity to wind direction (removing the need for yaw control systems and improving performance in atmospheric conditions with wind shear), their design which allows the heavy machinery to be at the base of the wind turbine (and therefore more easily maintained), and their absence of fatigue in the blades caused by cyclic gravitational loading (which becomes significant in large-diameter HAWTs).

However, the financial cost of the blades of VAWTs is typically more than the 
blades of a HAWT with an equivalent rotor swept area [2], and VAWTs experience low starting torque and are unable to self start at low wind speeds, and therefore require a starting system $[1,7]$. Due to the difference in torque produced by a VAWT blade when moving perpendicular to the wind and when moving parallel to the wind, the aerodynamic loading on the blades is cyclic [1]. Gearboxes and generators do not operate well with a highly varying torque, and the extruded aluminum which was used in the construction of the VAWT blades in the 1970s to 1990s suffered from poor fatigue resistance, resulting in premature failures of the VAWT blades [1]. Consequently, the vast majority of research into and development of wind turbines has been related to HAWTs since the mid-1990s [1].

During the 1990s and 2000s, the majority of research into wind turbines was focused on HAWTs. Recently, there has been a renewed interest in VAWTs due to their perceived suitability for urban and off-shore environments. In an urban environment, small VAWTs may provide a low-cost, low-footprint means to capture wind energy from highly variable winds.

In an off-shore environment, particularly in deep water regions, large VAWTs are more advantageous (and therefore more economical) than HAWTs with similar output because of the absence of cyclic gravitational loading on the blades, and a design which allows for the heaviest components of turbine (the electrical generator and gearbox) to be placed near or below the water's surface for a more stable configuration and easier operations and maintenance $[1,12]$.

With this renewed interest in use of VAWTs, it is important to be able to estimate the acoustic characteristics of the turbines during their design to minimize the annoyance to surrounding people and wildlife. At this time, the author has not been able to find any published work on the non-empirical prediction of the noise generated by VAWTs. 


\subsection{Motivation}

One of the obstacles to wind energy development is the noise from the turbines [13]. The noise created by VAWTs in urban environments has the potential to be an annoyance because of their proximity to people. The noise generated by large wind turbines, whether on-or off-shore, is typically dominated by infrasonic frequencies which cannot be heard by humans, but have been shown to be perceived as an annoyance [14] and are the cause for popular concerns about wind turbine operations contributing to the symptoms of vibroacoustic disease in people living near HAWTs $[15,16]$. The infrasound may also adversely affect local wildlife, such as whales which communicate using infrasound [17].

This noise is difficult to evaluate until the turbines are installed, and is a particular concern in regions near human populations [2]. As the aeroacoustics are considered to be a primary source of noise for VAWTs [2], it is important for the designer of VAWTs to be able to understand and predict, during the design phase, the noise which will be created by the turbine in order to ensure the noise levels are acceptable.

The present work creates a tool which is capable of predicting the aeroacoustic characteristics of VAWTs and provides important information about various VAWT designs and operating conditions, permitting the wind turbine designer to minimize the expected total noise emissions.

\subsection{Fundamentals of VAWTs}

Before proceeding, it is important to first understand the characteristics of Darrieus VAWTs and the parameters often used to describe them. 


\subsubsection{Theory of VAWT operations}

To understand how a Darrieus rotor operates, consider the two-dimensional scenario with a symmetrical airfoil of chord length $c_{\mathrm{T}}$ rotating at constant angular velocity $\boldsymbol{\Omega}_{\mathrm{T}}$ around a point at a given radius $r_{\mathrm{T}}$ where $c_{\mathrm{T}} \ll r_{\mathrm{T}}$, with the airfoil oriented in the direction of rotation. This airfoil represents the horizontal cross-section of a rotor blade.

A symmetric airfoil can be expected to have an angle of attack tangent to its path of motion when rotating through a still medium, and therefore create neither lift nor torque about the axis of rotation. In order to generate the lift and corresponding torque, consider the same rotating airfoil in a uniform flow, which represents the wind. This uniform flow causes the angle of attack to vary around the azimuth of the rotation, as shown in Fig. 1.2, and creates the necessary torque about the axis of rotation to sustain the turbine's operation.

The present work only considers vertically-oriented VAWTs in a uniform wind velocity. The spatial coordinate system used is centered along the axis of the VAWT, with the positive 1-direction oriented in the direction of wind velocity and the positive 3-direction aligned vertically upwards. The VAWTs considered in the present work all rotate in an anti-clockwise direction when viewed from above, such that the rotational velocity vector of the rotor $\Omega_{\mathrm{T}}$ is aligned with the positive 3 -direction. The azimuth angle is measured from the downwind side of the turbine in the direction of the rotor's rotation.

Fig. 1.2 is a schematic of the horizontal cross-section of a VAWT rotor's equator which shows the blade azimuth positions of $\theta_{\mathrm{T}}=0^{\circ}, 90^{\circ}, 180^{\circ}$, and $270^{\circ}$ for a rotor with a radius of $r_{\mathrm{T}}$. In this work, the azimuth angle is defined to be $0^{\circ}$ while immediately downwind. Each azimuth position shows the free-stream velocity vector $\mathbf{U}_{\infty}$, the negative kinematic velocity vector $-\mathbf{V}=-\boldsymbol{\Omega}_{\mathrm{T}} \times \mathbf{r}_{\mathrm{T}}$, the geometric angle of 


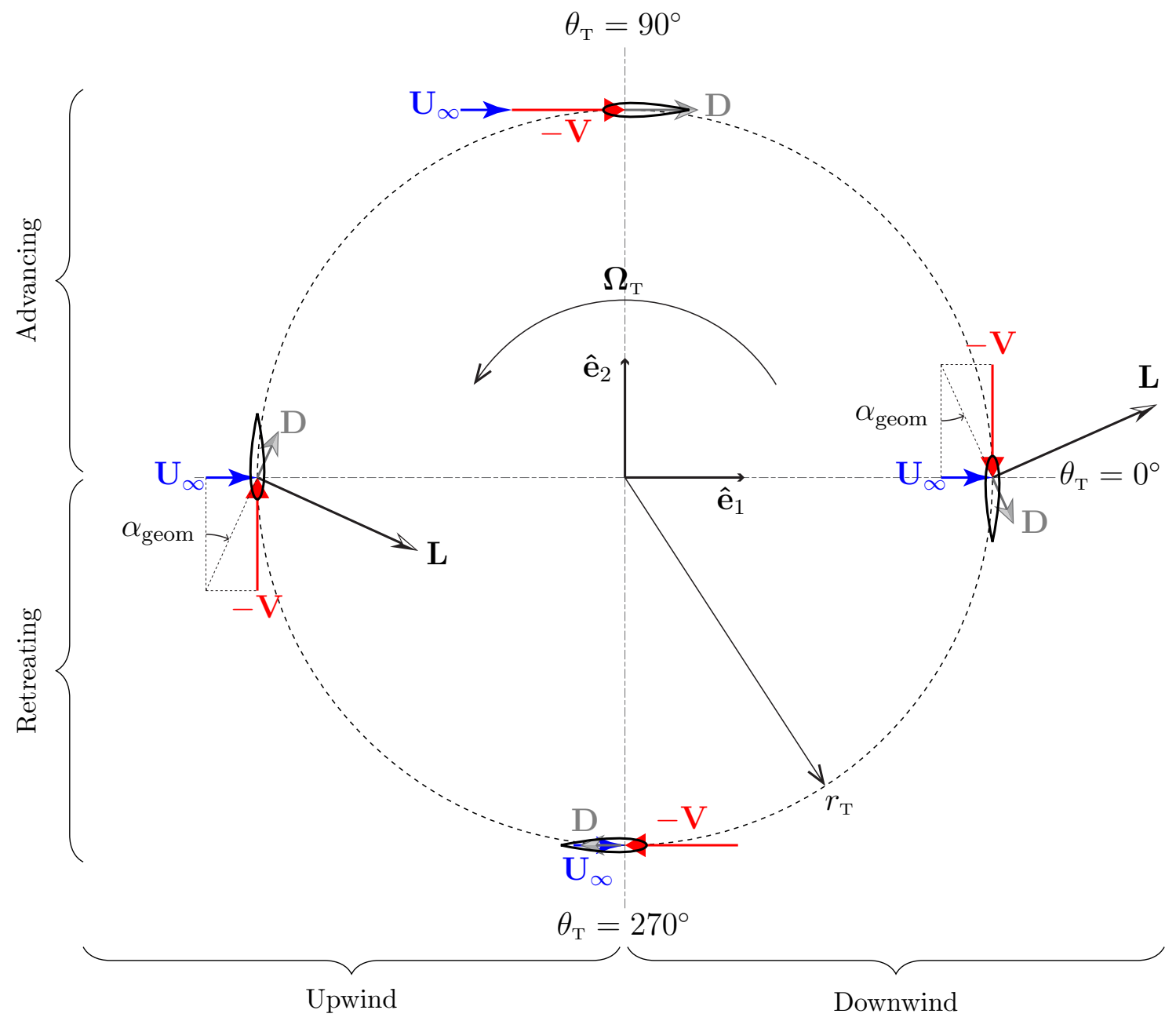

Figure 1.2: Top view of a horizontal-section of a VAWT with rotor blade positions $\theta_{\mathrm{T}}=0^{\circ}, 90^{\circ}, 180^{\circ}$, and $270^{\circ}$ shown

attack $\alpha_{\text {geom }}$, the lift vector $\mathbf{L}$, and the drag vector $\mathbf{D}$. Intervals of azimuth which are often used to describe the blade position are "upwind" for $\theta_{\mathrm{T}} \in\left(90^{\circ}, 270^{\circ}\right)$ or "downwind" for $\left(270^{\circ}, 360^{\circ}\right] \cup\left[0^{\circ}, 90^{\circ}\right)$, and "advancing" for $\left(0^{\circ}, 180^{\circ}\right)$ or "retreating" for $\left(180^{\circ}, 360^{\circ}\right)$. It is noted that other works may use different conventions than the one presented here.

The geometric angle of attack of a section of a rotor blade over the VAWT azimuth is highly dependent on the local ratio between kinematic velocity and free-stream velocity. A large speed ratio $(\gg 1.0)$ causes the angle of attack to vary by only 


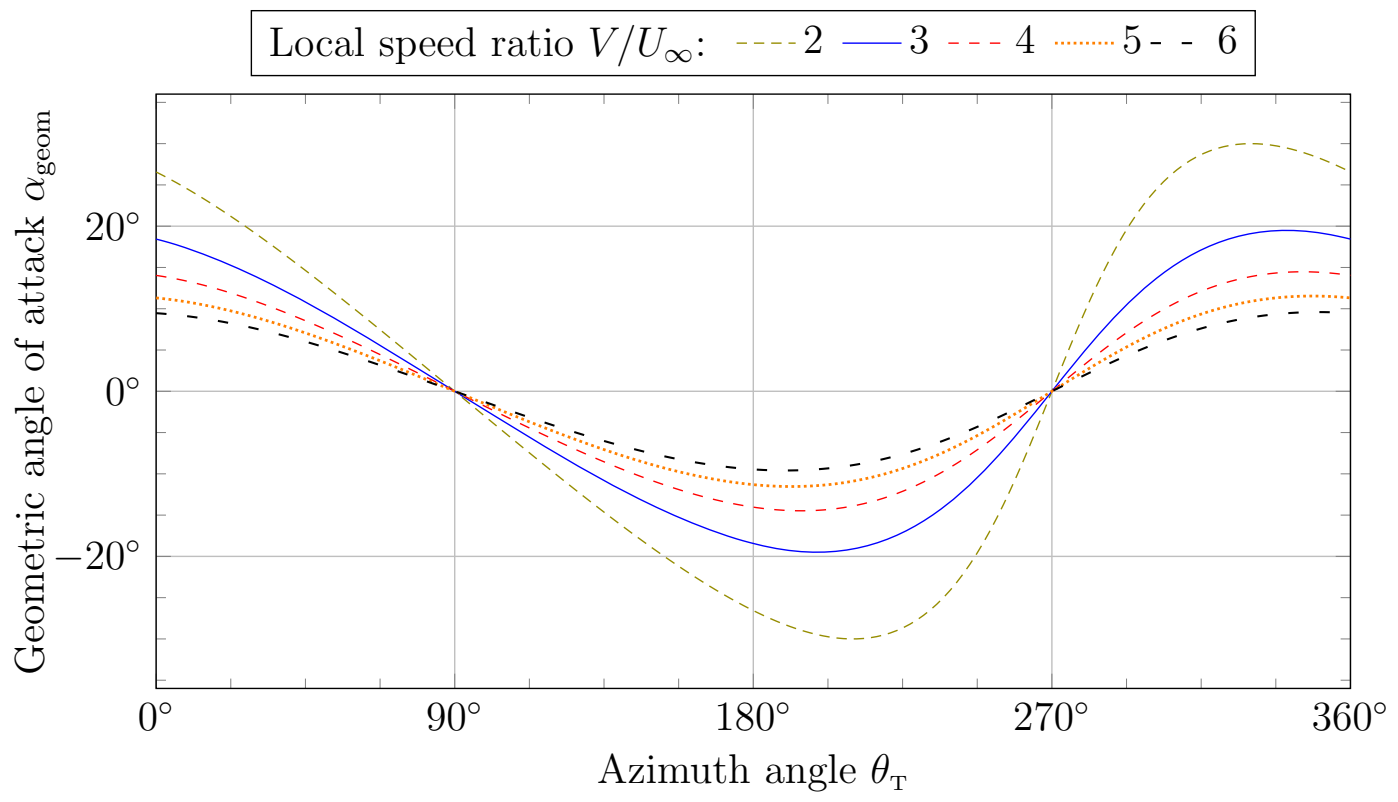

Figure 1.3: Plot of the geometric angle of attack of a section of VAWT blade over the rotation with various local speed ratios

a few degrees from zero in the negative and positive directions since the rotational component of velocity is larger than the free-stream velocity component. As the speed ratio approaches and becomes less than a value of 1.0, the angle of attack of the section will approach an angle of attack of $\pm 180^{\circ}$ when at an azimuth of $\theta_{\mathrm{T}}=270^{\circ}$ since the wind velocity component will be greater than the rotational velocity component. Fig. 1.3 shows this variation in angle of attack for different local speed ratio. This wide range of possible angles of attack is particularly problematic for rotors with a wide range of rotor radius along the blades' span, such as Troposkein VAWTs, leading to flow separation and stall phenomena near the ends of Troposkein rotor blades at moderate tip speed ratios (TSRs).

The rotor blades on a VAWT experience an undisturbed flow around them as curvilinear due to their curved path of motion. This relative curvilinear flow effectively creates a "virtual camber" on the blades, causing them to behave as though their airfoil cross-section were cambered with the opposite direction of curvature as 
the path of motion [18]. The curvilinear flow also introduces a virtual incidence angle which in turn adds a negative offset to the geometric angle of attack which makes the effective angle of attack more negative at upwind azimuth angles and less positive at downwind azimuth angles.

In addition to this unsteadiness, a VAWT rotor blade passes through the wake of other blades, the tower, and its own wake, thereby affecting the relative flow velocity experienced by the blades and the angle of attack. In fact, for a rotor blade in a region of wake with significant vorticity, angle of attack is no longer a useful measure of the flow around the blade as the flow is not uniform. These wake-body interactions are highly dependent on turbine's TSR, with the degree of interaction with rotor blade wakes increasing with TSR.

\subsubsection{VAWT parameters}

VAWTs are often characterized by many of the parameters which are used for HAWTs, including swept area, solidity ratio, height-to-diameter ratio, and TSR.

The swept area of a turbine is the frontal area which the turbine's rotor blades pass through. For a straight-bladed VAWT, this is simply the diameter multiplied by the rotor blade span.

The turbine rotor's solidity and height-to-diameter ratios are functions of the turbine's geometry only and are calculated respectively as [19]

$$
\begin{aligned}
\sigma_{\mathrm{T}} & =\frac{N_{b} c_{\mathrm{T}} L_{b}}{A_{\text {swept }}}, \\
\left(\frac{h}{d}\right)_{\mathrm{T}} & =\frac{h_{\mathrm{T}}}{2 r_{\mathrm{T}}},
\end{aligned}
$$

where $N_{b}$ is the number of rotor blades, $c_{\mathrm{T}}$ is the chord length of the blades (equal and constant along the span of each blade), $L_{b}$ is the total length of a single blade, 
$A_{\text {swept }}$ is the swept area, $r_{\mathrm{T}}$ is the rotor radius measured from the axis of rotation to the outermost point of the rotor blades, and $h_{\mathrm{T}}$ is the height of the rotor blades. Wind turbines are often characterized by their diameter $d_{\mathrm{T}}=2 r_{\mathrm{T}}$.

The TSR is the ratio between the rotational velocity of the outermost point of the rotor (the "tip") and the free-stream velocity (the wind). It is calculated as

$$
\lambda_{\mathrm{T}}=\frac{\Omega_{\mathrm{T}} r_{\mathrm{T}}}{U_{\infty}},
$$

where $\boldsymbol{\Omega}_{\mathrm{T}}$ is the angular velocity of the rotor and $\mathbf{U}_{\infty}$ is the wind velocity. For a straight-bladed VAWT with rotor blades parallel to the axis of rotation, the entire length of each blade is located at the tip radius $r_{\mathrm{T}}$ and therefore experiences local speed ratio equal to the TSR. For rotor blades spanning a range of radial positions, the local speed ratio is simply the ratio between the rotational velocity at the corresponding radial position and the free-stream velocity.

Forces on the rotor blades in the directions tangential and normal to the path of motion are commonly considered during analysis. The positive tangential direction is tangent to the path the rotating blade follows, and the positive normal direction is directed away from the axis of rotation. For a known net force $\mathbf{F}$ on a section of blade at an azimuth angle of $\theta_{\mathrm{T}}$, the tangential and normal force components are

$$
\begin{gathered}
\mathbf{F}_{\mathbf{T}}=\mathbf{F} \cdot\left(-\sin \theta_{\mathrm{T}} \hat{\mathbf{e}}_{\mathbf{1}}+\cos \theta_{\mathrm{T}} \hat{\mathbf{e}}_{\mathbf{2}}\right), \\
\mathbf{F}_{\mathbf{N}}=\mathbf{F} \cdot\left(\cos \theta_{\mathrm{T}} \hat{\mathbf{e}}_{\mathbf{1}}+\sin \theta_{\mathrm{T}} \hat{\mathbf{e}}_{\mathbf{2}}\right) .
\end{gathered}
$$

Non-dimensional forms of these forces are often used at various positions along 
the span of the blades, including

$$
\begin{aligned}
& F_{T}^{+}=\frac{2 F_{T}}{\rho_{0} A_{\mathrm{sec}} U_{\infty}^{2}}, \\
& F_{N}^{+}=\frac{2 F_{N}}{\rho_{0} A_{\mathrm{sec}} U_{\infty}^{2}},
\end{aligned}
$$

where $A_{\text {sec }}$ is the one-sided area of the section of blade being considered.

In Fig. 1.2, it can be seen that aerodynamic drag primarily contributes to the tangential force in the negative direction. Lift can similarly be seen to contribute primarily to the normal force, though it also contributes to the tangential force in the positive direction. The contribution of drag to the normal force is negligible compared to lift for large lift-to-drag ratios.

\subsection{State of the art}

Aeroacoustics is the study of sound generated aerodynamically due to unsteady flow and its interactions with structures immersed in the flow [20,21]. Accurate predictions the aeroacoustic sound therefore require an accurate representation of the aerodynamics of the flow. This section outlines the current state of the art in aeroacoustics and aerodynamics applicable to VAWT operations.

\subsubsection{Aeroacoustics}

Scientific interest in aeroacoustics, which is the study of the sound created by airflows, first started around the early 1950s when the noise generated by jet engines became a concern. Lighthill was a pioneer in the field of aeroacoustics, and his acoustic analogy [22] later formed the basis of many works in the field of aeroacoustics. Lighthill's acoustic analogy applies to a finite region of rotational flow in an unbounded fluid, and 
transforms the Navier-Stokes and continuity equations to form an exact, inhomogeneous wave equation, with source terms having importance only within the turbulent region $[22,23]$. The sound created by this unbounded fluid is called "aerodynamic sound".

Many practical sound generation problems, such as VAWTs, involve moving boundaries or moving sources interacting with such boundaries [21]. Two approaches to solving such problems include the Kirchhoff and Ffowcs Williams-Hawkings (FWH) formulations.

The Kirchhoff formulation is an inhomogeneous wave equation with a distribution of acoustic sources over a surface which encloses all physical sources [24]. This "Kirchhoff surface" must be placed in the linear flow region, which is not well defined. The Kirchhoff formulation has been shown to be unreliable in practice for aeroacoustic problems if the surface is not placed carefully or if wakes pass through the surface [25].

The FW-H method uses an equation which is an exact rearrangement of the continuity equation and the Navier-Stokes equations into the form of an inhomogeneous wave equation with distributions of two surface source terms and one volume source term $[24,26]$. This so-called FW-H equation distinguishes between the three source distributions, including the thickness and loading surface sources and the quadrupole volume source, which each have a distinct physical meaning. The noise generated by the displacement of fluid is captured by the thickness source, and the noise generated by the unsteady force distribution over the body surface is captured by the loading source [25].

The quadrupole source represents the nonlinearities due to the local sound speed variation and the finite particle velocity near the surface [25]. The quadrupole sources require significant computational effort to calculate, and are often neglected since the 
thickness and loading sources account for the majority of the acoustic for subsonic flow fields [25]. A variation of the FW-H method which involves a penetrable data surface encompassing the body surfaces and all quadrupole sources producing non-negligible sound allows for the calculation of quadrupole effects without volume integration. However, high-resolution computational fluid dynamics (CFD) is required in the nearfield region to optimize the location of the data surface [27].

The FW-H equation has been successfully applied to a wide variety of problems, including propellers, helicopter rotors, and HAWTs [28-31]. For surfaces moving at subsonic speeds through a medium at rest, solutions of the FW-H equation called Formulations 1 and 1A were derived by Farassat and Succi are commonly used [27,28,32]. An observer time derivative which must be determined numerically in Formulation 1 lead to the development of Formulation 1A, which replaces the observer time derivative with a source time derivative which may be calculated numerically or analytically, and is now recommended by Farassat over Formulation 1 [27].

Formulation 1A does not capture the convective effects associated with a medium with a uniform velocity, such as wind. A third formulation, called Formulation 1C, has been derived by Najafi-Yazdi et al. [33] as an extension to Formulation 1A which also takes the contribution of a uniformly moving medium into account. As VAWTs require wind to operate, the associated convective effects should be taken into account. If the velocity of the medium is zero, Formulation $1 \mathrm{C}$ becomes equivalent to Formulation 1A [33]. Other approaches to predicting the acoustics of moving sources in moving mediums have been successfully implemented, such as the work by Wells and Han for propeller noise [29], but are not as generic and well-presented as the work by Najafi-Yazdi.

While sound waves are compressions and rarefactions of the medium with oscillations of pressure and density, solving for the acoustic characteristics in space using 
conventional CFD methods is impractical. Obstacles to using CFD approaches include: high velocity of the flow structures requiring very fine time resolution, acoustic pressure fluctuations being orders of magnitude smaller than the aerodynamic pressure field, and dispersion and dissipation errors associated with the spatial and temporal resolutions. Because of these obstacles and the broad frequency range of interest in acoustics, most purely numerical CFD schemes are inferior to codes based on the FW-H equation in terms of both accuracy and efficiency [34].

Brooks et al. [35] described five mechanisms of airfoil self-noise, which are due to the interactions between an airfoil blade and the turbulence produced in its own boundary layer and near wake. These include turbulent-boundary-layer-TE noise, laminar-boundary-layer-vortex-shedding noise, separation-stall noise, TE-bluntnessvortex-shedding noise, and tip vortex formation noise. While the contributions of these mechanisms are not independently solved for, a comprehensive acoustic formulation should take the acoustic sources of all mechanisms into account. These mechanisms are important to the broadband (random) noise, but may also be tonal (harmonic) in character $[13,35]$.

Casper and Farassat [36] described another solution of the FW-H equation called Formulation 1B which computes the loading noise from an unsteady surface pressure distribution on a thin airfoil in the time domain and has been shown to be capable of predicting the broadband noise spectrum of an airfoil interacting with homogeneous turbulence and seems to suitably predict the trailing edge noise [34].

Few investigations into the aeroacoustics of VAWTs have been performed. The most notable works include the measurements by Kelley et al. [37] of the 17-metre Troposkein VAWT constructed by Alcoa for Sandia Laboratories and the 24-metre NRC/DAF Troposkein VAWT, and Pearson's [5,38] experimental and semi-empirical modelling of an straight-bladed model VAWT. 


\subsubsection{Aerodynamics}

An accurate aerodynamic representation is required to proceed with predicting the aeroacoustics of a problem. For implementations of the FW-H equation, the velocity distribution over the surface of the bodies is required to determine the thickness sources, and the pressure distribution over the same surface is required to determine the loading sources.

As the primary goal of this work is to investigate the acoustic characteristics of VAWTs, reasonably accurate pressure and velocity distributions should be obtained without excessive computational cost. Empirical results based on steady uniform flows are unsatisfactory due to the unsteady nature of VAWTs (including the effective camber described in Sec. 1.3.1), the high degree of wake-body interaction, and complex three-dimensional VAWT geometry.

Several methods have been used for predicting the aerodynamic performance of VAWTs, many of which were developed in the 1970s and '80s do not require great computer processing power. The most common of these methods include single and multiple streamtube momentum models, free- and fixed-wake vortex models, and high-speed lifting line models $[2,39]$.

Streamtube momentum models are the simplest and most limited in their scope, only providing performance results for the turbine as a whole. Vortex models are more complex and are better suited to predicting the near-wake structure of the rotor and the forces on the rotor blades [2,39].

Vortex models involve representing the span-wise sections of the blades as discrete bound vorticities, whose strengths are determined from the circulation related to the amount of lift created by that blade element (as determined by the local angle of attack and experimental data for the airfoil) [39,40]. A vortex lattice structure, composed of span-wise and chord-wise vortex filaments with uniform strengths determined by 
Helmholtz's vorticity theorems and Kelvin's circulation theorem, is released from the TE of the blades and convected downstream $[2,39,41]$. The vorticity of each of the vortex filaments induces a flow velocity along the blade sections, which are used in determining the angle of attack and flow velocity experienced by each section.

Following the approach of vortex models, experimental results for the pressure distribution over the blade sections may be determined based on the incident flow velocity and angle of attack. However, this data would not capture the unsteady effects due to the curved path the blades follow, would not be accurate in regions with significant wake-induced vorticity, and is not readily available for many airfoils over an angle of attack range as great as that typically experienced by VAWT blades with small local speed ratios.

Computational fluid dynamics (CFD) solvers provide a means to obtain very detailed information about the entire flow field around geometries in unsteady flow, such as those surrounding VAWTs. CFD solvers, such as those based on finite volume methods or finite element methods, may be used to predict the wake effects, the flow separation over the blades, and the highly three-dimensional flow experienced by VAWTs. However, solving the entire flow field in detail around a VAWT using CFD would require moving meshes and obtaining accurate results require a significant amount of computational time [42].

Since detailed information about the flow around the VAWT is not the main concern of this work, a method which is concerned primarily with solving the pressure distribution over the surface geometry is preferred. Panel methods are a numerical form of the boundary integral equation method which can discretize arbitrary bodies and their wake into distributions of surface singularity elements (or panels). Pioneered by Hess and Smith in the 1960s and '70s [43-45] for solving the pressure distribution 
over aircraft, panel methods have been successfully implemented on a range of situations including inviscid two- and three-dimensional, steady and unsteady, subsonic and supersonic flows over thin and thick bodies [46].

Extensions to the panel methods have allowed for modelling unsteady aerodynamics, viscous effects such as flow separation, and stall [46]. In free-wake methodologies, the wake panels may be convected at the local flow velocity after being released from the body panels to model the wake. While traditional panel methods represent the wake as panels of singularity distributions, the solution may become unstable when body panels encounter the wake panels due to their regions of singularity and/or discontinuity. Stable solutions may be achieved by incorporating methods to remove these numerical instabilities, which involve removing any flow singularities. Vortex lattice methods, such as the work by Dixon [42], and vortex particle methods with cut-off lengths are two possible solutions. For example, the Generalized Unsteady Vortex Particle method (GENUVP) developed at the National Technical University of Athens has successfully used a surface panel method and vortex particle wake model to predict the aerodynamics and aeroacoustics of helicopter rotor blades [30].

A number of hybrid methodologies exist and have been applied to Darrieus VAWTs, such as the vorticity transport model which combines a finite-volume model of the wake with a lifting-line model for the blade aerodynamics [3], but are beyond the scope of this work. Further discussion on the aerodynamic modelling of wind turbines can be found in [40].

\subsection{Structure of the thesis}

The present chapter contains an introduction to VAWTs including a brief history, a description of how they function, the flow conditions experienced by the rotors, 
and existing approaches to predicting the aeroacoustics and aerodynamics typical of VAWTs.

Chapter 2 describes the aerodynamic analysis methodology used to predict the distribution of aerodynamic quantities necessary for aeroacoustic analysis over lifting surfaces such as wings and rotor blades. A verification of the implementation involving a comparison of the results to accepted aerodynamic solutions for certain test cases is presented.

Chapter 3 similarly describes the methodology used for aeroacoustic analysis and verifies the results using three fundamental test cases.

Chapter 4 attempts to validate the aerodynamic and aeroacoustic results for VAWTs. Several studies the effects of various VAWT geometric and operational parameters on the aerodynamic and aeroacoustic characteristics of the VAWT are presented with a discussion of the results.

Chapter 5 summarizes the major conclusions of this work and presents recommendations for future work. 


\section{Chapter 2}

\section{Aerodynamic Analysis}

\subsection{Aerodynamic methods}

The approach taken by this work to modelling the aerodynamics of the problem is a hybrid unsteady inviscid panel method with vortex particles. This approach involves representing the inflow as a uniform laminar flow with a specified velocity, the VAWT rotor blades as combined source and doublet panels over the surface of the blades, the near wake of the rotor blades as doublet panels released from the TE of the blades, and the far wake as freely convected vortex particles. The current work neglects incoming atmospheric turbulence, geometric features other than the rotor blades, and viscous flow phenomena. The rotor blades are considered to be rigid.

A time-marching scheme is used for the VAWT rotation, with the rotor rotating at a constant prescribed angular velocity. The VAWT torque output is used to determine when the aerodynamic solution over the surface of the rotor blades has reached a steady-state. 


\subsubsection{Singularity elements}

The aerodynamic methods used in this work employ a variety of singularity elements which are distributions of basic inviscid elementary flows. The elementary flows which are of concern include point sources/sinks, point doublets, and point vortices. The singularity elements which are composed of distributions of these elementary flows include source panels, doublet panels, vortex filaments, and vortex particles.

\section{Elementary flows}

A point source is a point emanating fluid in all directions where all the streamlines are straight lines originating from the point [47]. In two-dimensional space, the curves of constant velocity potential form concentric circles around the point and the velocity decreases by the inverse of the distance to the point source. In three-dimensional space, the surfaces of constant velocity potential form concentric spheres around the point and the velocity decreases by the inverse of the square of the distance to the point source. The velocity in both cases is proportional to the strength of the point source. A point "sink" is simply a point source with a negative strength, and draws fluid into a point rather than emanate fluid from the point.

A point doublet is created when a point source and a point sink of equal but opposite strengths are brought infinitesimally close together. The point doublet can be represented by a vector whose magnitude is equal to the strength of the source/sink pair multiplied by the separation distance, and whose direction is from the sink towards the source.

A point vortex may also be represented by a vector, where fluid is being rotated around the vector according to the right-hand-rule convention. Similar to a point source, the velocity at which the fluid travels is proportional to both the strength of the vortex and the inverse of the distance to the vortex. 


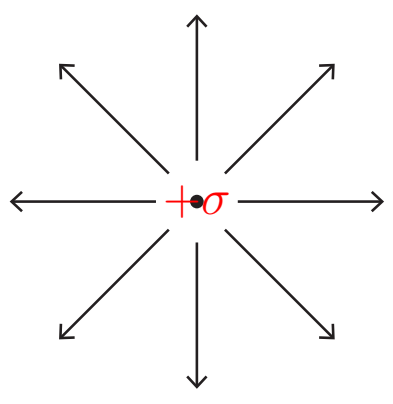

(a) Point source (positive)

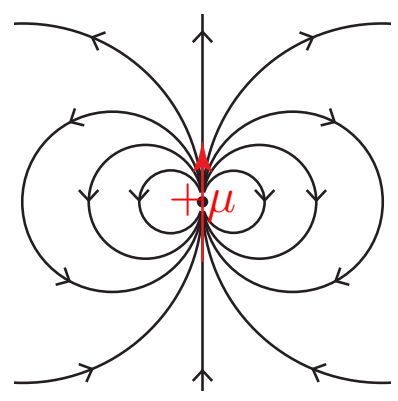

(b) Point doublet (positive upwards)

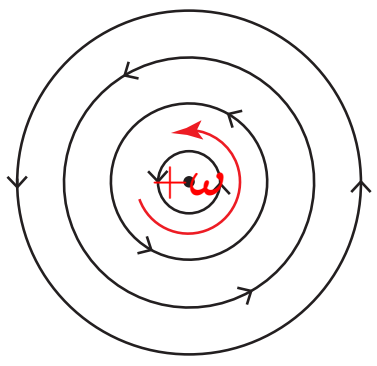

(c) Point vortex (positive out of page)

Figure 2.1: Illustrations of a point source, point doublet, and point vortex in two-dimensions

\section{Panel singularity elements}

The geometry of panel singularity elements in two- and three-dimensions may be first-order (flat), second-order (quadratic), or a higher order. Flat first-order panels are used most often, but care must be taken for three-dimensional applications where flat panels may not align properly with surrounding panels. Higher order panels introduce greater complexity to the problems and do not have solutions for some integral equations, and are therefore rarely used.

The distribution of singularity strength over a panel may be uniform, linearly varying, or of higher order over the element. For uniform-strength singularity distributions, the boundary conditions (BCs) of the panels are typically imposed at a certain point (the "control point") on the panel.

Source panels are singularity elements characterized by a continuous distribution of point sources over a line (2D) or a surface (3D). The velocity potential at position q due to a source panel defined by the surface $S$ is calculated as

$$
\Phi_{\sigma}(\mathbf{q})=\frac{1}{4 \pi} \int_{S} \sigma \frac{1}{\mathbf{r}} d S
$$

where $\mathbf{r}$ is the distance from $\mathbf{q}$ to a point on the panel and $\sigma$ is the source strength 
density (often abbreviated to just "source strength") corresponding to that point on the panel.

Similarly, a doublet panel is a continuous distribution of point doublets over a curve (2D) or a surface (3D), where the direction of the doublet strength at any point on the curve/surface is normal to the curve/surface on one side. The velocity potential at position q due to a doublet panel defined by the surface $S$ is calculated as

$$
\Phi_{\mu}(\mathbf{q})=\frac{-1}{4 \pi} \int_{S} \mu \frac{\partial}{\partial \hat{\mathbf{n}}}\left(\frac{1}{\mathbf{r}}\right) d S,
$$

where $\mathbf{r}$ is the distance from $\mathbf{q}$ to a point on the panel, $\mu$ is the doublet strength density (often abbreviated to just "doublet strength") corresponding to that point on the panel, and $\hat{\mathbf{n}}$ is the unit normal vector for the point on the panel in the direction for positive doublet strengths.

The velocity induced by these panels is found using the gradient of the scalar velocity potential

$$
\mathbf{u}^{\prime}(\mathbf{q})=\nabla \Phi(\mathbf{q})
$$

and is visualized in Fig. 2.2 using streamlines and arrows with a color gradient from blue (small) to red (large) for the streamlines and arrows to represent the velocity magnitude. Larger arrows also indicate larger velocity magnitudes.

The equations used for velocity potential and perturbation velocity around firstorder uniform-strength source and doublet panels in three-dimensions have been derived and presented in $[43,44,46]$. For completeness, App. A contains these equations as they are used in the present work, with several corrections to the equations from [46] noted. 


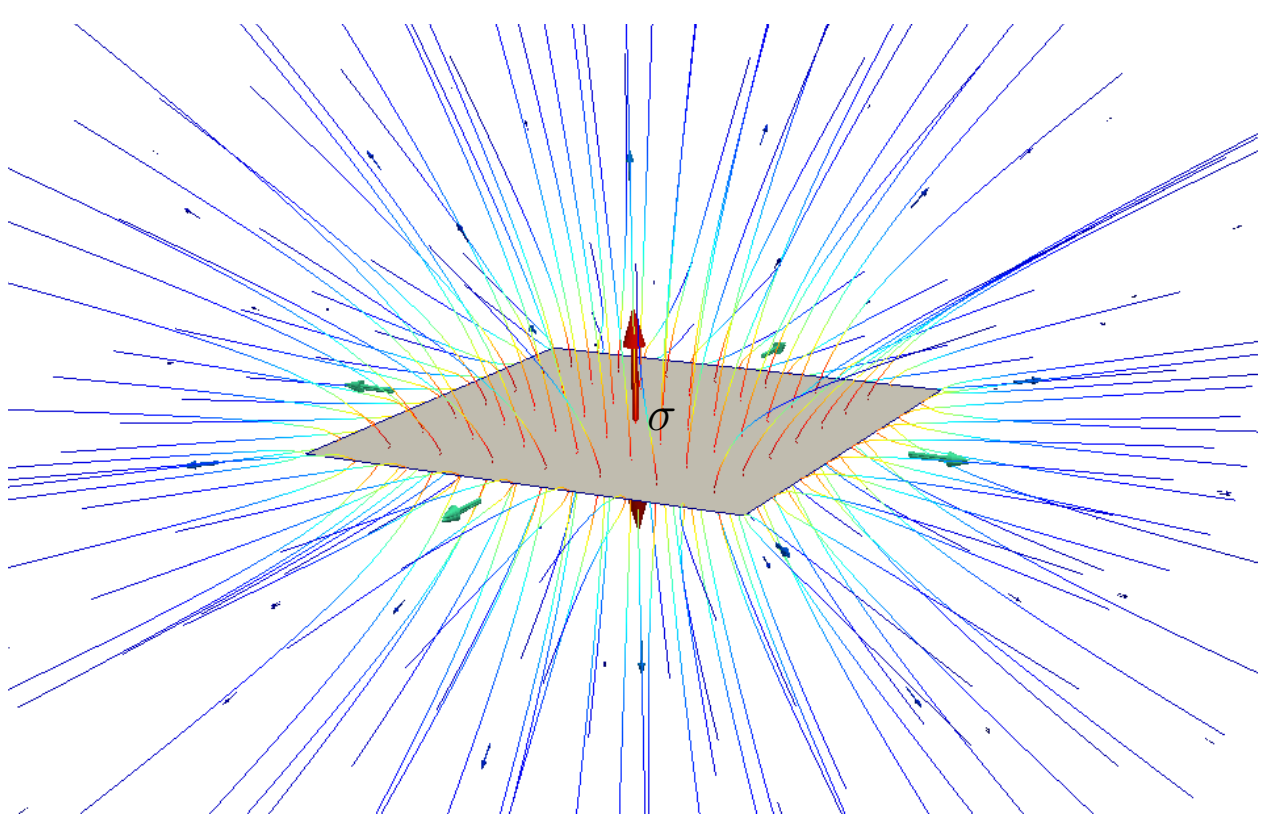

(a) Uniform-strength quadrilateral source panel

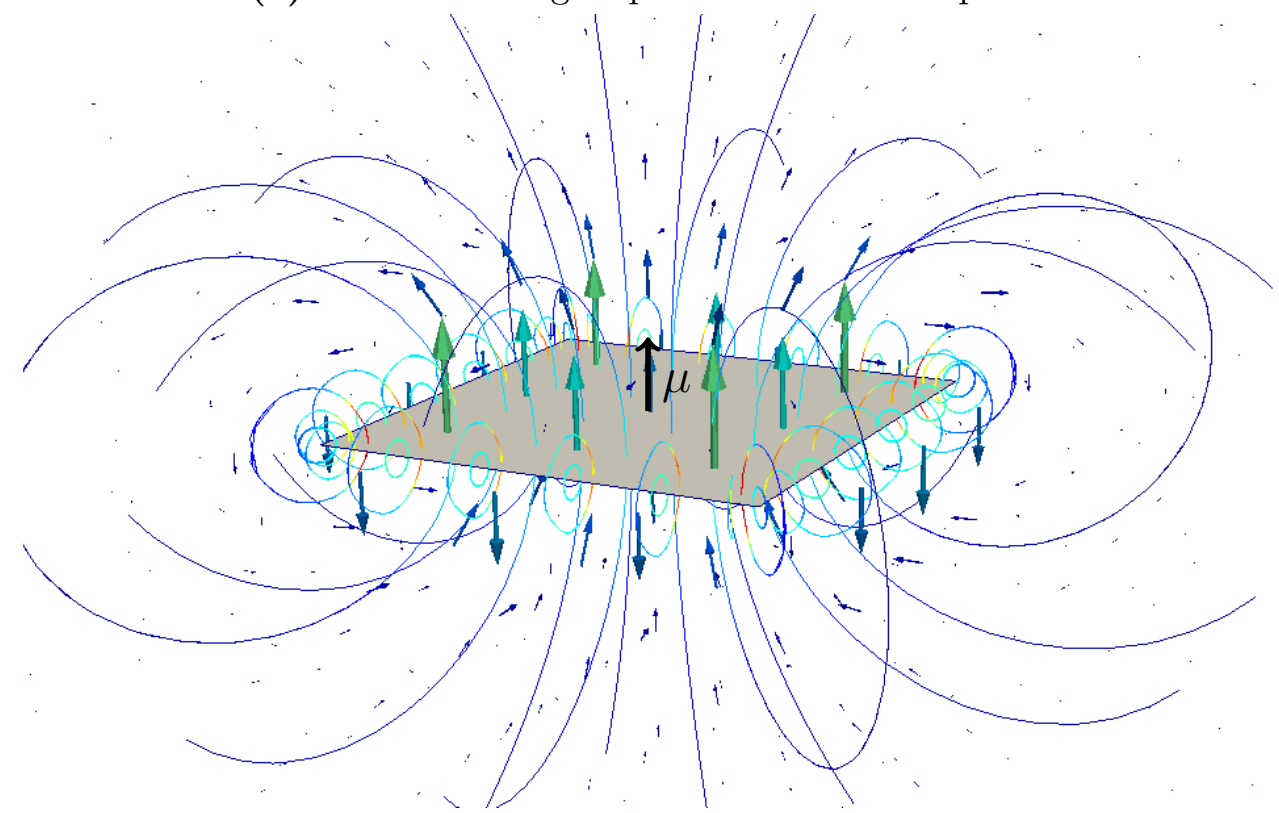

(b) Uniform-strength quadrilateral doublet panel

Figure 2.2: Streamlines and arrows representing velocity fields around uniform-strength quadrilateral panels 


\section{Three-dimensional vortex singularity elements}

Vortex filaments are three-dimensional distributions of point vorticities along a threedimensional curve, or "filament", where the vorticity is tangent to the filament along the entire length. Vortex filaments in this work are considered to be linear with uniform vorticity along the length of the filament.

It is important to note that a discontinuity in a distribution of doublet strength is equivalent to a vortex filament along the edge of discontinuity which has a strength equal to that of the discontinuity [46]. Therefore, a lone doublet panel with uniform strength $\mu$ is equivalent to a vortex ring (a closed vortex filament) coincident with the edge of the panel which has a uniform strength of $\mu$.

The final flow singularity to note in this work is that of a "vortex particle" or "vortex blob". A vortex particle (VP) is a point vortex in two- or three-dimensional space which has a position $\mathbf{Z}$, an intensity of $\boldsymbol{\xi}$, and a smoothing radius or cut-off length $\epsilon$. The velocity induced at position $\mathbf{q}$ by a VP at position $\mathbf{Z}$ is found using [48]

$$
\mathbf{u}_{\mathrm{VP}}^{\prime}(\mathbf{q})=\frac{\boldsymbol{\xi} \times \mathbf{r}}{4 \pi r^{3}} \zeta_{\epsilon}(r)
$$

where

$$
\begin{gathered}
\mathbf{r}=\mathbf{q}-\mathbf{Z}, \\
\zeta_{\epsilon}(r)=1-e^{-(r / \epsilon)^{3}} .
\end{gathered}
$$

The regularizing or "smoothing" function $\zeta_{\epsilon}(r)$ and the smoothing radius $\epsilon$ are introduced to remove the velocity singularity at position $\mathbf{Z}$. Without the inclusion of this smoothing, $\lim _{r \rightarrow 0} \mathbf{u}_{\mathrm{VP}}^{\prime}(\mathbf{q})=\infty$. This smoothing helps make the VP more physical as it captures the viscous diffusion effects in the core of a vortex [49]. The velocity due to a vortex particle is illustrated in Fig. 2.3 for different smoothing radii, and the three-dimensional velocity field is visualized in Fig. 2.4. 


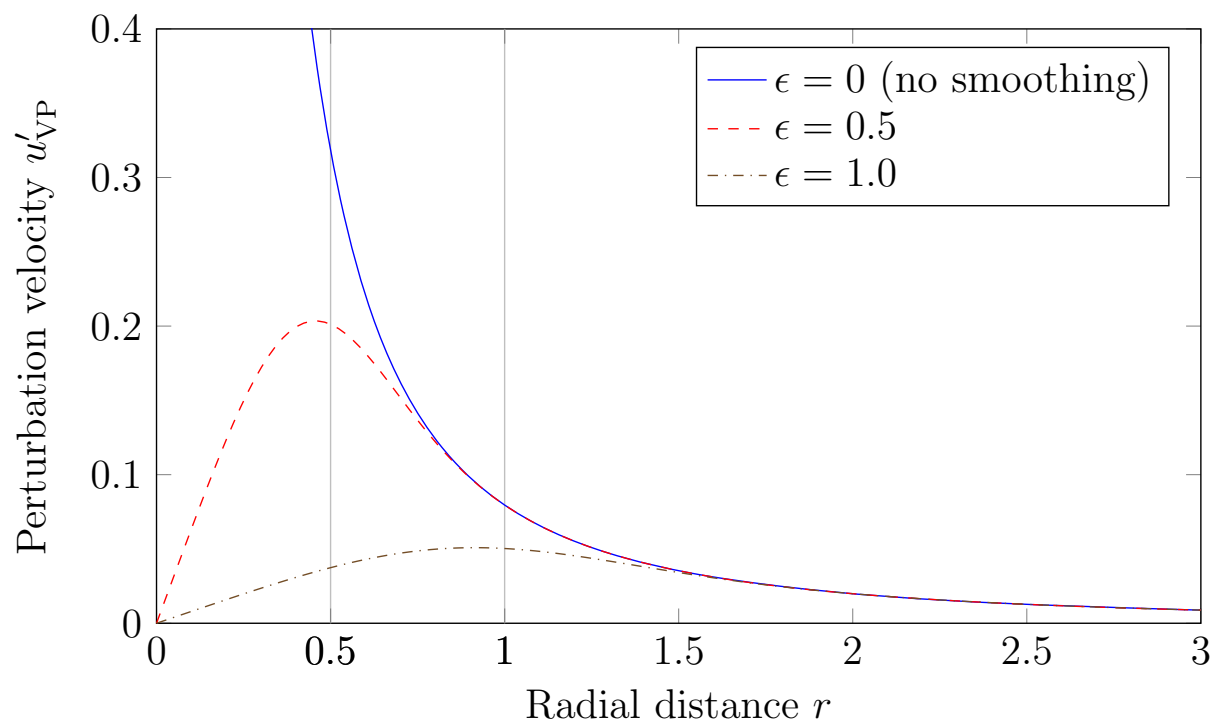

Figure 2.3: VP perturbation velocity as a function of radial distance from the VP, where $r=|\mathbf{r}|,|\boldsymbol{\xi}|=1$, and $\boldsymbol{\xi} \perp \mathbf{r}$

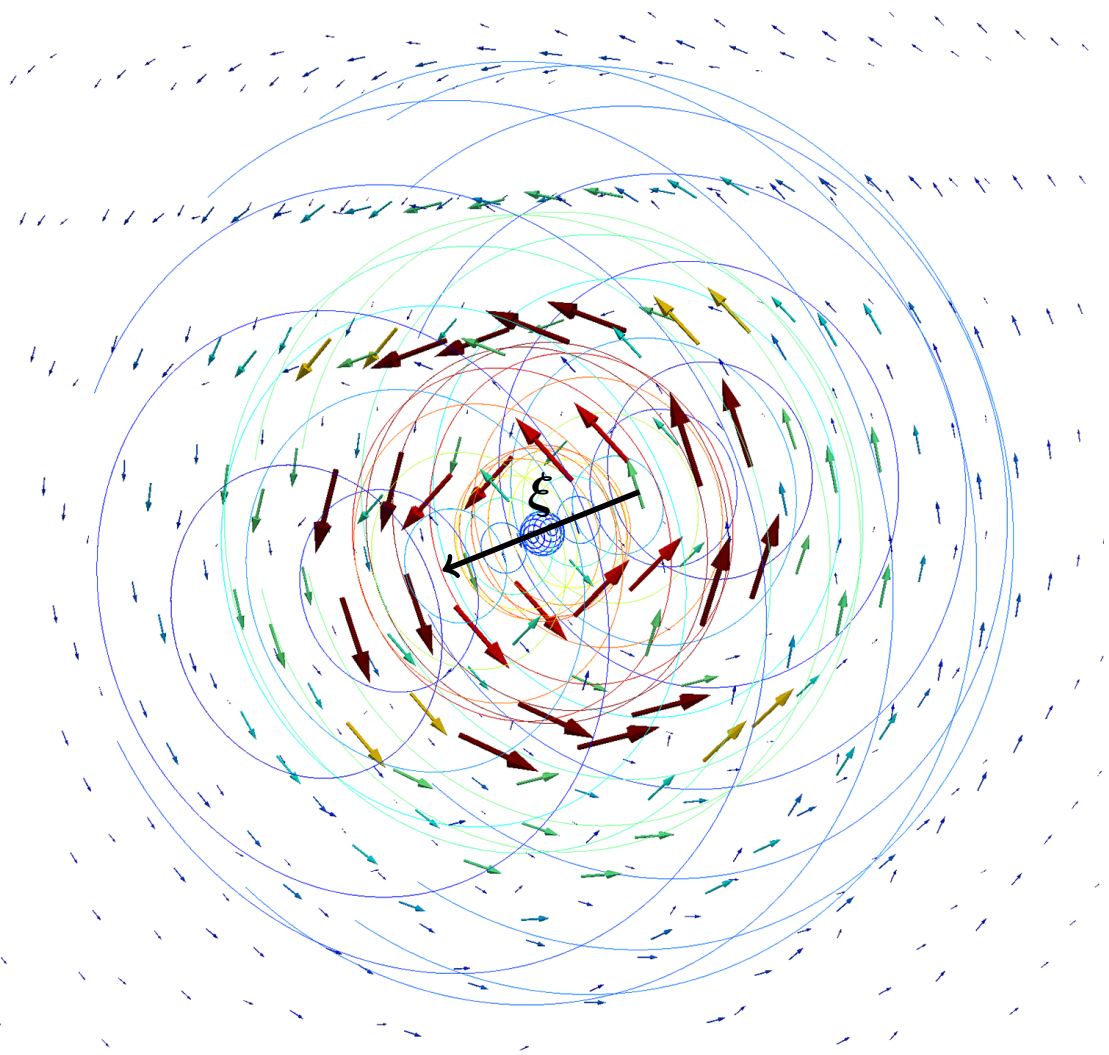

Figure 2.4: Streamlines and arrows representing the velocity field around a VP with a non-zero smoothing radius 
The smoothing radius gives the vortex particle the alternate name "vortex blob" as it can be thought of to have a spherical volume with radius $\epsilon$ associated with it. In this thesis and other existing literature, the terms "vortex particle" and "vortex blob" are interchangeable, while "vortex particle" is more common in existing literature.

It is noted that there are a number of approaches to regularizing the vortex particle, though the approach taken here with $\epsilon$ and $\zeta_{\epsilon}(r)$ in Eq. (2.4c) has been used in similar works such as those by Beale and Majda [49] and Voutsinas [48].

\subsubsection{Geometric representation}

The present work uses quadrilaterals (first-order panels with four sides) to approximate the surface of the VAWT blades. Using a set of $\left(N_{a}\right)$ two-dimensional airfoil coordinates which define the constant cross-sectional profile of the blades, and a blade profile containing a series of $\left(N_{h}+1\right)$ elevations and radial positions (which define the blade to have $N_{h}$ span-wise sections), the points defining the blade geometry are created. The first and last coordinates of the airfoil profile must be coincident to properly implement the Kutta condition with the current approach [46]. Each surface panel has a unit outer normal vector $\hat{\mathbf{n}}$ which is positive in the direction away from the body.

Fig. 2.5 illustrates the chord-wise and span-wise discretization parameters for a two-bladed Troposkein rotor with $N_{a}=10$ and $N_{h}=9$. Fig. 2.5a further illustrates the control points (located at the centroid of each panel) and the outer unit normal vectors. The values used for discretization must be investigated to ensure adequate refinement, as in Sec. 4.1.

The distribution of panels should be such that there is a denser concentration of panels in regions with greater curvature or where aerodynamic effects are expected to vary significantly in space. While the actual panels generated to represent a rotor 


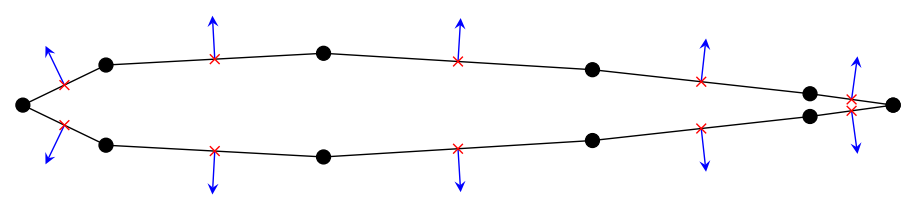

- Panel endpoints $\times$ Control points $\longrightarrow$ Outer unit normal vectors

(a) Geometric discretization of a NACA 0012 airfoil using ten chord-wise $\left(N_{a}=10\right)$ for illustration purposes

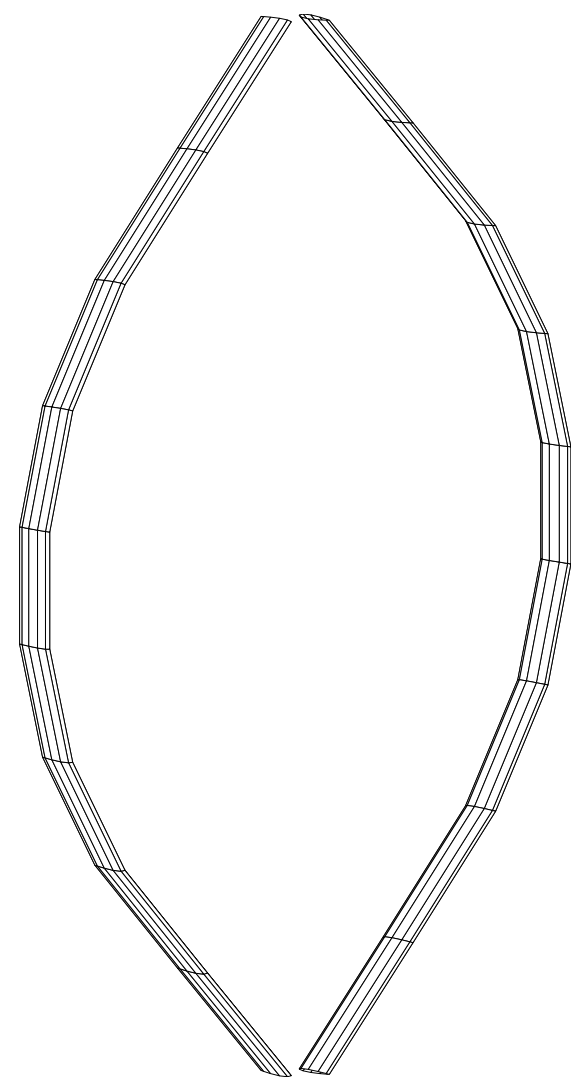

(b) Geometric discretization of a two-bladed Troposkien rotor using nine span-wise sections $\left(N_{h}=9\right)$ and ten chord-wise sections $\left(N_{a}=10\right)$ for illustration purposes

Figure 2.5: Illustration of a geometric discretization of a Troposkien rotor with a NACA 0012 airfoil 
blade may not always be perfectly co-planar (a requirement to be a quadrilateral), the span-wise discretization of the blades may be increased to maximize the co-planarity of the panel's bounding points.

The central tower, supporting guy-wires, and ground are not included in the present geometric representation. The wake from the shaft of a model VAWT has been observed to not have any significant impact on the acoustic radiation from the VAWT rotor [5], supporting the exclusion of the tower from the geometric representation.

\subsubsection{Wake representation}

The Kutta condition is a condition imposed on the flow at the TE of an airfoil section to obtain results which are representative of the physical flow around the airfoil $[46,47]$. One approach to satisfying the Kutta condition is prescribing the vorticity along the TE to be zero. The inclusion of a wake panel with uniform doublet strength which is released from the TE of every airfoil section provides a means to apply the zero-vorticity Kutta condition while also including the effects of shed spanwise and chordwise vorticity from each section. However, in situations with any degree of strong body-wake interactions such as VAWT rotor blades passing through the wake of other rotor blades, special care must be taken to avoid numerical instabilities associated with the wake representation.

For steady aerodynamic problems, the wake panels may be prescribed to extend from the TEs with very long or semi-infinite length $[46,50]$. Since the aerodynamics of VAWTs are unsteady, a new row of finite-length wake panels is prescribed at each time step, including the length and orientation of each panel. The wake representation from the previous time step, including wake panels, vortex particles, and vortex filaments, is convected by the local flow velocities. 


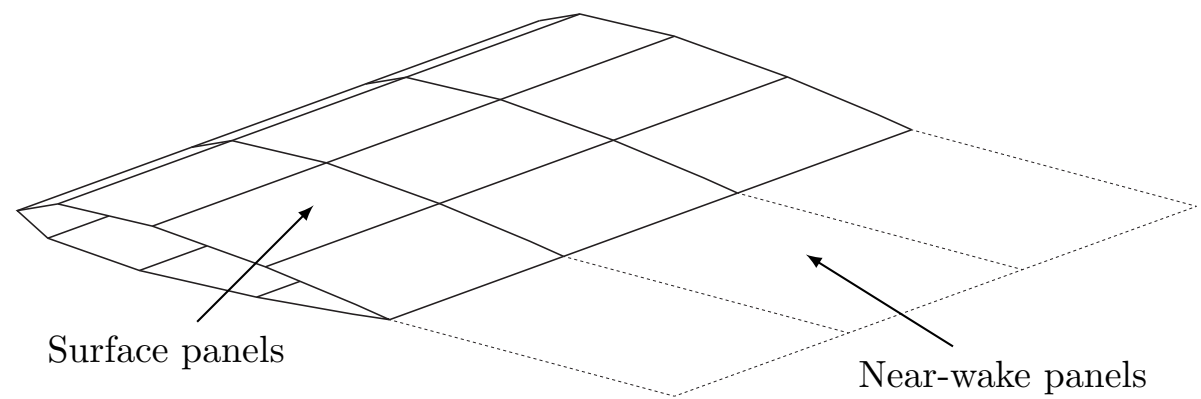

Figure 2.6: Illustration of a single near-wake panel row for a wing with $N_{h}=3$

The approach taken in this work divides the wake into two regions: the nearwake, and the far-wake. The near-wake includes the most recently shed row of wake panels represented using a grid of uniform strength doublet panels. After every time step, the row of near-wake panels is convected then converted to vortex filaments and grid-free vortex particles, thereby becoming part of the far-wake.

\section{Near-wake representation using doublet panels}

The near-wake for a blade with $N_{h}$ span-wise sections contains an equal number of uniform-strength quadrilateral doublet panels, as shown in Fig 2.6. While the leading edge of the row of near-wake panels must be coincident with the TE of the blade, the placement of the TE (and therefore overall shape) of the wake panels is less constrained.

Placement of the TE of wake panels requires both a distance and relative angle from of the TE of the blade. An ideal placement accurately reflects the true shape of the wake being shed from the blade TE and captures the unsteady effects as a continuously varying strength wake would.

Options for prescribing the relative angle of wake panels include: parallel to the free-stream velocity, along the bisector of the blade TE, or along the path of motion followed by the TE. Fig. 2.7 illustrates these positions. More complex methods involve 

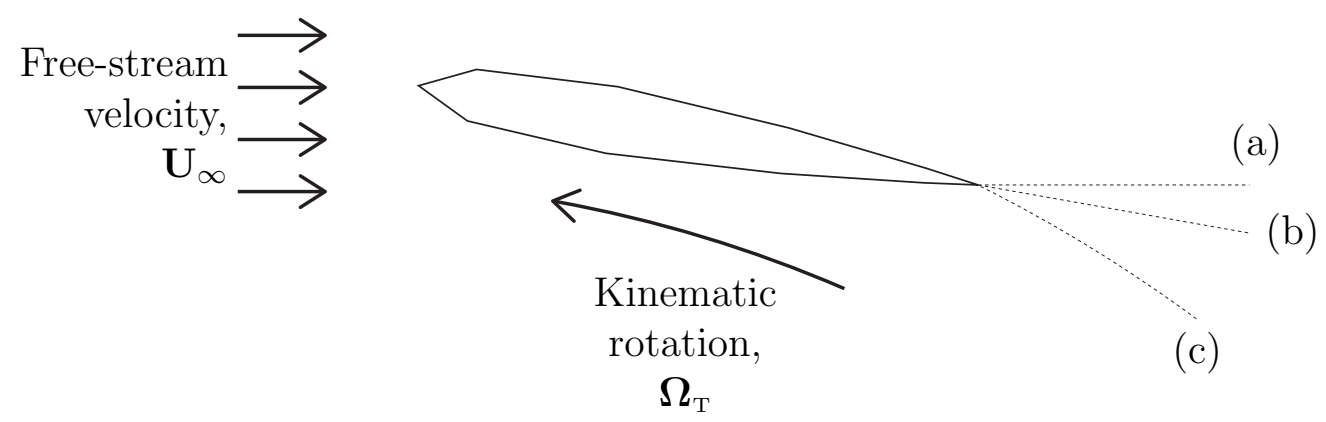

Figure 2.7: Curves illustrating the positioning of the TE of the near wake using:

(a) free-stream velocity, (b) bisector angle of $\mathrm{TE}$, and (c) kinematic rotation

iterative schemes which seek the position of the TE of the row of near-wake panels which best satisfy the zero pressure jump form of the Kutta condition exist, but are very costly for $3 \mathrm{D}$ implementations [42].

The present work includes options for aligning the near-wake panels with the bisector of the blade TE, with the freestream velocity, or with the path of motion the blade TE follows. An additional option superimposes the displacements associated with the free-stream velocity and motion of the TE over the previous time step.

The distance of between the TE of the near-wake panel row and the TE of the blade is related to the distance covered by a fluid particle, released from the blade TE, during the time interval between the previous and current time steps. A typical approach is to place the TE of the near-wake panel row $20 \%$ to $30 \%$ of aforementioned distance to better represent a continuously varying wake strength $[42,46]$. The present work provides the option to specify the distance as a fraction of this distance, or as a fraction of the blade's chord length.

With the shape of the near-wake panels known, the zero-vorticity form of the Kutta condition may be considered. Recalling that uniform-strength doublet panels are equivalent to vortex rings coincident with the border of the panel and of equal strength to that of the uniform doublet strength, three sets of vortex filaments can 
be thought to exist at the TE of the blade: the TE portion of the surface doublet panel at the bottom of the TE, the TE portion of the surface doublet panel at the top of the TE, and the portion of the near-wake doublet panel coincident with the blade's TE.

To satisfy the Kutta condition, zero-vorticity at the blade's TE is achieved by setting the near-wake panel strength equal to

$$
\mu_{\mathrm{wake}}=\mu_{\mathrm{top}}-\mu_{\mathrm{bottom}},
$$

where $\mu_{\text {bottom }}$ is the strength of the doublet panel at the bottom of the TE, $\mu_{\text {top }}$ is the strength of the doublet panel at the top of the TE, and $\mu_{\text {wake }}$ is the strength of the near-wake doublet panel shed from the section of TE shared by the other two panels.

\section{Far-wake representation using vortex particles}

While pure panel method approaches may be used for unsteady flows, singularities associated with the panels may be encountered in problems with strong body-wake interaction.

When using the velocity potential of wake doublet panels to obtain a solution, the discontinuity across the panel proves to be problematic when a wake panel crosses body surface panels. Similarly, when using the velocity induced by wake doublet panels, the singularity in velocity along the edges of the wake doublet panels creates instabilities in the solution as the edges of the wake panels approach the body panels. Since a VAWT blade interacts very strongly with the wake originating from other blades as well as its own wake, the wake from VAWT blades must be represented in a way which will be both physically reasonable and numerically stable. 
The program developed in the present work can represent the far-wake as uniformstrength doublet panels, and include their perturbation velocity field into the solution using a Neumann BC in order to investigate their appropriateness for VAWT applications (Sec. 2.1.4 contains additional details on the implementation of this BC). Due to the aforementioned issues which arise with the uniform-strength doublet panel representation of the wake, an alternate method of representation of the far-wake is required.

The approach taken to modelling the far-wake for the majority of this work is to represent it using vortex particles. This approach has been used successfully in conjunction with panel methods to predict the aerodynamics of rotorcraft [30], and has well-known optimization schemes. The details of this approach may be found in App. A.3 along with schematics showing the conversion of near-wake panels to far-wake vortex filaments and vortex particles.

After the near-wake panels are convected away from the trailing edge, the vorticities along three of the four edges of the each panel are converted to a vortex particle with a vorticity and position which conserves the zero and first moments of vorticity $\boldsymbol{\omega}[48]$ :

$$
\begin{aligned}
\boldsymbol{\xi}(\tau) & =\int_{S_{k}} \boldsymbol{\omega}(\mathbf{y} ; \tau) \mathrm{d} S, \\
\boldsymbol{\xi}(\tau) \times \mathbf{Z}(\tau) & =\int_{S_{k}} \boldsymbol{\omega}(\mathbf{y} ; \tau) \times \mathbf{y} \mathrm{d} S,
\end{aligned}
$$

where $S_{k}$ includes the area of the entire convected near-wake panel $k$ and the three edges which are not coincident with the trailing edge of the new near-wake panels. There is only non-zero vorticity $\boldsymbol{\omega}$ along the edges of the uniform-strength doublet panels, which allows the integrations in Eq. (2.6) to become summations over three uniform-strength vortex filaments. 
The fourth edge of each convected wake panel which was not included in the integration is treated as a vortex filament. On the following time step, when the next row of near-wake panels is convected and converted into vortex particles, the previous vortex filament strengths are included in the TE of the convected wake panels during the integration in Eq. (2.6). For further details on the transformation of near-wake to far-wake, refer to App. A.3.

Determining an appropriate smoothing radius for the vortex particles is not as straight-forward. A too-large smoothing radius, and the vortex particle's effects will be weakly dispersed over a large area. A too-small smoothing radius, and the vortex particle's effects will be concentrated to a small region, increasing the likelihood of instability in the solution.

Lee and $\mathrm{Na}$ [51] used a smoothing radius of $10 \%$ of the chord length for simulating helicopter rotor blades, though the reasoning for this decision could not be found. The author of this work considers it reasonable that the smoothing radius should be a function of both the fluid's viscosity as well as the size of the geometry being considered. In the present implementation, the smoothing radius may be specified as a fraction of chord length, the wake panel's average length, average width, or the square root of the panel's area. An investigation of a varying smoothing radius is not presented in this work. The author has done limited investigations into the effects, and it was found that a smoothing radius equal to the square root of the panel's area is acceptable.

The vortex particles are convected and deformed at every aerodynamic simulation time step using first-order differentiation. The rates of convection and deformation for a vortex particle with position $\mathbf{Z}_{p}$ and intensity $\boldsymbol{\xi}_{p}$ are determined from the material 
character of the vortex particle using [48]:

$$
\begin{gathered}
\frac{\mathrm{d} \mathbf{Z}_{p}(\tau)}{\mathrm{d} \tau}=\mathbf{u}\left(\mathbf{Z}_{p}, \tau\right), \\
\frac{\mathrm{d} \boldsymbol{\xi}_{p}(\tau)}{\mathrm{d} \tau}=\left(\boldsymbol{\xi}_{p}(\tau) \nabla\right) \mathbf{u}\left(\mathbf{Z}_{p}, \tau\right),
\end{gathered}
$$

where the local fluid velocity $\mathbf{u}$ is the sum of the free-stream velocity and the perturbation velocities

$$
\begin{aligned}
\mathbf{u} & =\mathbf{U}_{\infty}+\mathbf{u}_{\text {solid }}^{\prime}+\mathbf{u}_{\text {near-wake }}^{\prime}+\mathbf{u}_{\text {far-wake }}^{\prime} \\
& =\mathbf{U}_{\infty}+\left(\mathbf{u}_{\sigma}^{\prime}+\mathbf{u}_{\mu}^{\prime}\right)+\left(\mathbf{u}_{\mu_{w}}^{\prime}\right)+\left(\mathbf{u}_{\mathrm{VF}}^{\prime}+\mathbf{u}_{\mathrm{VP}}^{\prime}\right) .
\end{aligned}
$$

\subsubsection{Solving the panel method}

The VAWT rotor blades are represented by panels with both source strengths, which represent the thickness effects of the blades, and doublet strengths, which impose a net circulation around the bodies.

Two types of BCs may be used with to solve for the panel strengths. Neumann BCs require zero normal velocity at the control point of each surface panel whereas Dirichlet BCs use a constant-velocity potential method at the same points [46]. Solutions which use Dirichlet BCs have been shown to require less computational effort [46], and are therefore better suited for an application such as the simulation of VAWT rotor blades which requires solving for the panel strengths at hundreds or perhaps thousands of time steps.

The solution for a combined uniform source and doublet panel approach is not unique, thereby requiring either the source or doublet strengths to be specified [46]. In this work, the source strength of each panel is specified such that the inner potential 
(that is, the velocity potential inside the blades) is equal to the velocity potential of the free-stream and far-wake, resulting in

$$
\sigma_{j}=-\hat{\mathbf{n}}_{j} \cdot\left[\mathbf{v}-\left(\mathbf{U}_{\infty}+\mathbf{u}_{\text {far-wake }}^{\prime}\right)\right]_{j},
$$

where $\sigma_{j}$ is the uniform source strength of panel $j, \hat{\mathbf{n}}_{j}$ is the outer unit normal vector of panel $j$, and the sum of velocities in the square brackets is the velocity at which panel $j$ is moving through the local flow.

Before solving for the doublet panel strengths using the Dirichlet BCs, the velocity potential effects that each panel has on all other panels must be determined. Influence coefficients are a measure of these effects for panels with unit strengths and are organized into matrices $C_{i j}$ for the surface doublet strengths and $B_{i j}$ for the surface source strengths, where

$$
\begin{gathered}
B_{i j}=\frac{\Phi_{\sigma j}\left(\mathbf{y}_{i}\right)}{\sigma_{j}}=\Phi_{\sigma j}\left(\mathbf{y}_{i} ; \sigma_{j}=1\right), \\
C_{i j}=\frac{\Phi_{\mu j}\left(\mathbf{y}_{i}\right)}{\mu_{j}}=\Phi_{\mu j}\left(\mathbf{y}_{i} ; \mu_{j}=1\right) .
\end{gathered}
$$

Following this notation, $C_{i j}$ is the relative influence that the doublet strength of surface panel $j$ will have on the control point of surface panel $i$, and $B_{i j}$ is the relative influence that the source strength of surface panel $j$ will have on the control point of surface panel $i$. The doublet panel has a discontinuity of velocity potential along the panel's surface requiring the specification of each doublet panel's influence on itself to be

$$
C_{i i}=0.5 \text {. }
$$

The Kutta condition is incorporated into the doublet influence coefficients using 
the form of the Kutta condition from Eq. (2.5), resulting in the new doublet influence coefficients [46]

$$
A_{i j}= \begin{cases}C_{i j}, & \text { if } j \text { is not at the TE } \\ C_{i j}-C_{i w}, & \text { if } j \text { is at the bottom of the TE and sheds wake panel } w \\ C_{i j}+C_{i w}, & \text { if } j \text { is at the top of the TE and sheds wake panel } w\end{cases}
$$

where $C_{i w}$ is the influence coefficient of wake panel $w$ on the control point of surface panel $i$.

Following the constant-potential Dirichlet BCs methods in [46], the internal perturbation velocity potential of the body may be set to be zero. This allows for the unknown strengths of the surface doublet panels, $\mu_{j}$, to determined by solving the system of equations

$$
A_{i j} \mu_{j}=-B_{i j} \sigma_{j}
$$

Once the surface doublet strengths are known, the strengths of the near-wake doublet panels are calculated using Eq. (2.5).

\subsubsection{Aerodynamic post-processing}

The velocity and pressure distributions over the surface panels are required to calculate the thickness and loading acoustic source distributions. The velocity at each panel control point, relative to the panel, is a combination of the total perturbation velocity, the wind velocity, and the kinematic velocity, and is calculated using the same velocity equations as before.

Following a procedure similar one presented in [46], the gauge pressure distribution $p^{\prime}$ is calculated using the relative velocity $V$ and velocity potential $\Phi$ with the unsteady 
Bernoulli equation

$$
\frac{\partial \Phi}{\partial \tau}+\frac{V^{2}}{2}+\frac{p^{\prime}}{\rho_{0}}=\frac{p_{\infty}}{\rho_{0}} .
$$

The coefficient of pressure is the non-dimensionalized form of the gauge pressure, and can be calculated along a surface as

$$
C_{p}=\frac{p^{\prime}-p_{\infty}}{(1 / 2) \rho_{0} V_{\text {ref }}^{2}}=1-\frac{V^{2}}{V_{\text {ref }}^{2}}-\frac{2}{V_{\text {ref }}^{2}} \frac{\partial \Phi}{\partial \tau},
$$

where $V_{\text {ref }}$ is the reference velocity magnitude selected to be

$$
V_{\mathrm{ref}}=\Omega_{\mathrm{T}} r_{\mathrm{T}}+U_{\infty}
$$

\subsection{Aerodynamic component implementation}

As the aerodynamic simulations can be very computationally intensive, the aerodynamic component has been implemented as a program developed using FORTRAN 90. The aerodynamic simulations proceed by incrementally increasing the source time, and can be described as:

1. Read program input from text files

2. Generate geometry

3. Calculate $B_{i j}$ and $C_{i j}$

4. Place the near-wake panels

5. Calculate $C_{i w}$ and $A_{i j}$

6. Solve for the panel source strengths

7. Solve for the panel doublet strengths 
8. Solve for the pressure distribution

9. Export results for the present time step

10. If the simulation is not complete:

(a) Proceed to next time step

(b) Convect and deform the wake

(c) Update the geometry

(d) Place new near-wake panels

(e) Convert the previous near-wake panels into VPs

(f) Return to step 5

The simulation ends when all time steps designated by the program input are completed. Simulations of VAWT rotors are assumed to rotate at constant speed, and are advanced in time using an incremental increase of azimuth angle, where

$$
\Delta \tau=\frac{\Delta \theta_{\mathrm{T}}}{\Omega_{\mathrm{T}}}
$$

Time steps based an increment of azimuth angle are useful for rotating geometries as it will provide an equal number of time steps per revolution, regardless of the rotational velocity.

The surface influence coefficients $B_{i j}$ and $C_{i j}$ do not change in time for the rigid geometry, as the position of the surface panels remains constant in the local coordinate systems of other surface panels.

The number of surface panels, $N$, and the number of vortex particles, $N_{\mathrm{vP}}$, are the primary factors in determining the computational cost of a single time step. The computational cost of the aerodynamic simulation increases by an order of $N^{2}$ and 
by an order of $N_{\mathrm{VP}}{ }^{2}$ since the effects of the each panel and VP on every other panel and VP must be considered. While $N_{\mathrm{VP}}$ is not related to the airfoil discretization, it is proportional to the number of blades, the number of span-wise sections per bade, and the number of time steps. Increasing any of these parameters will therefore significantly increase the computational time required to obtain a solution.

The majority of the computational time is spent convecting and deforming the wake. To reduce the time required to complete the simulations, OpenMP is used to implement multi-core parallelization. Files exported from the program are in binary format, which performs significantly faster than exporting to ASCII format and results in much smaller file sizes.

MATLAB is used for post-processing the results, and The Visualization Toolkit software is used to visualize and animate the aerodynamic simulation in three dimensions. Visualization of the convection of vortex particles proves to be an effective means to understand the wake developed by geometry.

\subsection{Aerodynamic model verification and valida- tion}

To ensure the aerodynamic code in the present method works properly, several simple test cases are considered to verify that the methods have been implemented properly and produce the expected results. These cases include:

(A) the pressure distribution over a two-dimensional airfoil section;

(B) the span-wise distribution of lift over a wing in steady flow; and

(C) the lift of an airfoil undergoing oscillations in pitch. 


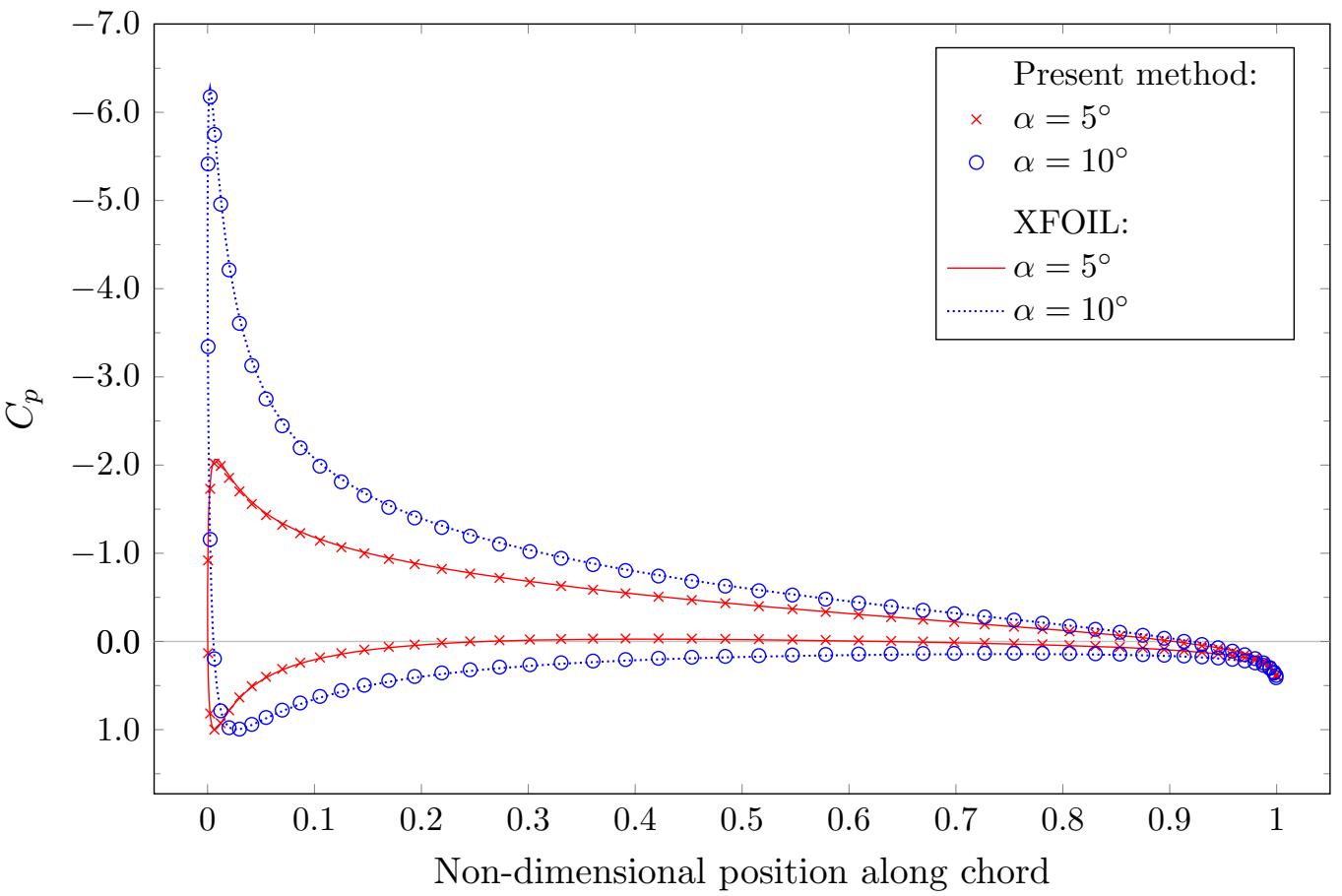

Figure 2.8: Coefficient of pressure distributions for a NACA 0012 airfoil at $\alpha=5^{\circ}$ and $\alpha=10^{\circ}$

The pressure coefficient distribution over two-dimensional airfoils is typically published for a range of angles of attack and Reynolds numbers. As the present method considers the flow to be inviscid, the Reynolds number will not impact the results, and the results at high angles of attack will not capture the effects of stall. Since the present method is a 3D implementation, Case (A) is addressed by modelling a high aspect-ratio wing $(R=100)$. Fig. 2.8 shows the distribution of pressure coefficient over a NACA 0012 airfoil at angles of attack of $5^{\circ}$ and $10^{\circ}$, with the present method producing results which are in excellent agreement with the subsonic airfoil software XFOIL [52], thereby verifying the steady two-dimensional behaviour of the present method. 


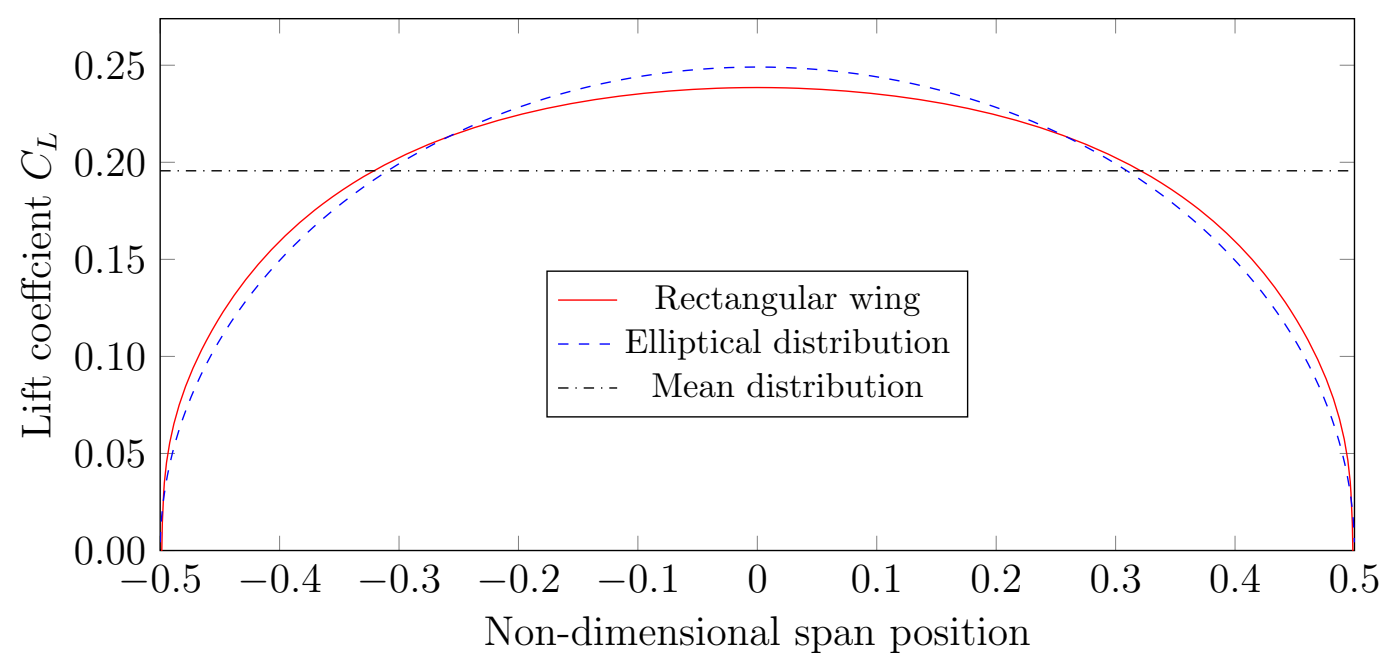

Figure 2.9: Span-wise distributions of lift coefficient along a wing in steady flow

To verify the three-dimensional aspects of the present method, Case (B) investigates the distribution of lift coefficient over the span of a rectangular wing. The lift over a wing is expected to be greatest at the center, and fall off to zero at the tips. Fig. 2.9 shows the span-wise distribution of the lift coefficient over an untwisted rectangular wing with an aspect ratio of $R=3.87$ and a NACA 0012 airfoil at an angle of attack of $3^{\circ}$. Fig. 2.9 also shows the mean value of lift coefficient, and the corresponding elliptical lift distribution profile. As expected, the lift distribution for the rectangular wing is similar to the elliptical distribution, and verifies the threedimensionality of the present method.

Case (C) considers a pitching airfoil to verify and validate the present method's modelling of unsteady aerodynamics. A wing with a NACA 0012 airfoil and aspect ratio of 100 is considered to oscillate in pitch about its quarter-chord axis, with the pitching motion described by $\alpha=3^{\circ}+10^{\circ} \sin \omega \tau$ with reduced frequency of $\omega c / 2 U_{\infty}=0.1$ 


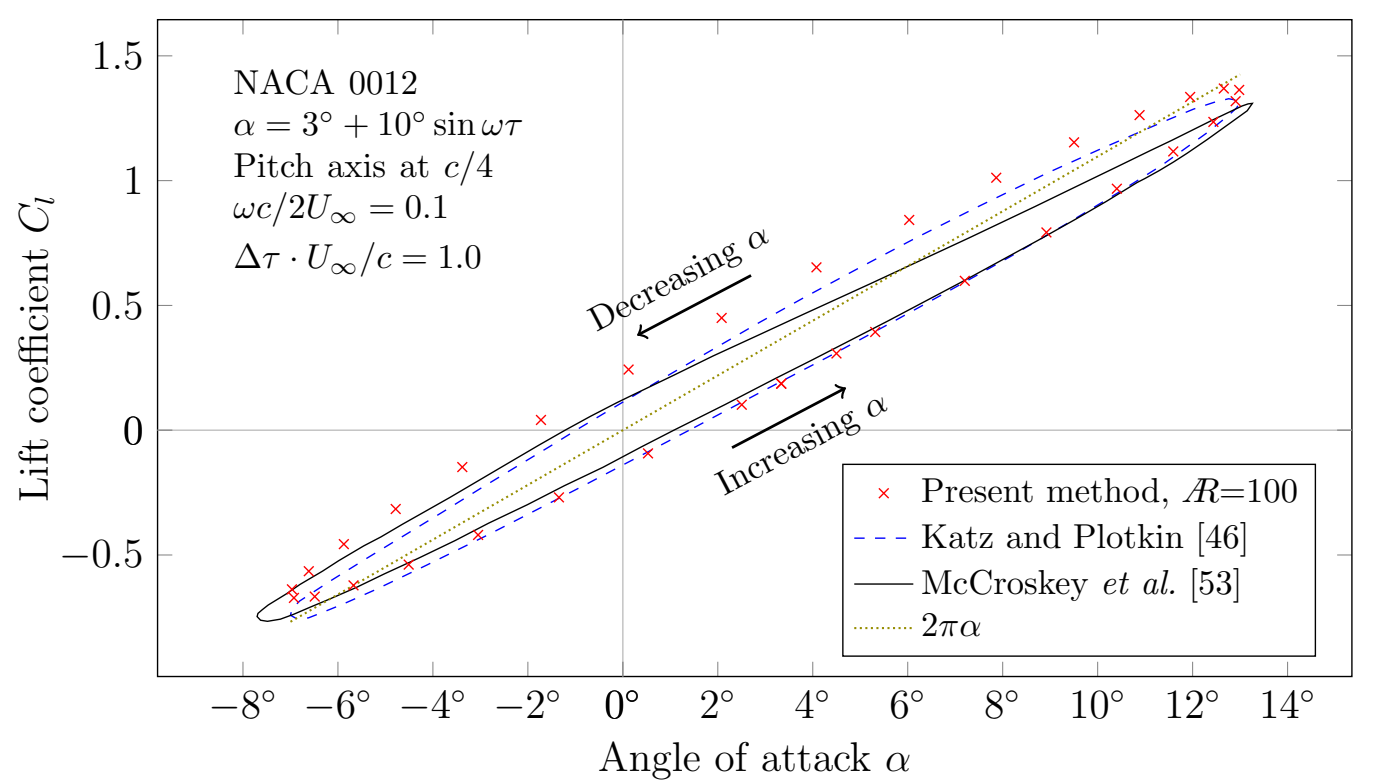

Figure 2.10: Unsteady lift coefficient for a pitching oscillation of a NACA 0012 airfoil

Fig. 2.10 shows lift coefficient hysteresis loop for the results obtained using the present method, Katz and Plotkin's [46] unsteady panel method results, and McCroskey's [53] experimental results. All results are in good agreement, and closely correspond to the lift-curve slope of $2 \pi \alpha$. The results from the present method and Katz and Plotkin do not capture the decreased lift coefficient at high and decreasing angle of attack, which was observed by McCroskey, as they are unable to capture the flow separation effects. Fig. 2.11 visualizes the vortex particles shed from the oscillating wing with the VPs shown at $1 / 4$ scale relative to the wing and with darker shades representing stronger vortex intensity.

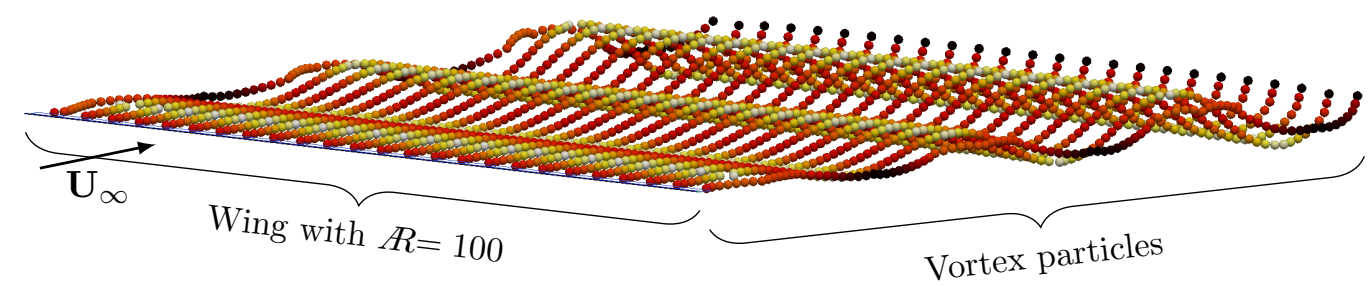

Figure 2.11: VPs shed from an oscillating wing 


\section{Chapter 3}

\section{Aeroacoustic Analysis}

Using the current methodology, once the aerodynamics of the problem are known, the aeroacoustic analysis may be performed. This chapter describes the method used for aeroacoustic analysis with verification using fundamental test cases.

\subsection{Aeroacoustic concepts}

Sound in a fluid is characterized by fluctuations in density of the medium, with a proportional fluctuation in pressure. These fluctuations are propagated away from the acoustic source throughout the fluid as a succession of compressions and rarefactions called sound waves [21].

Acoustic sources are often characterized into three groups: monopoles, dipoles, and quadrupoles. A monopole point source is a model for a pulsating sphere which radiates sound equally in all directions, and can therefore be represented using a fluctuating scalar value. A dipole is composed of two monopoles fluctuating outof-phase with equal strengths, separated by a small distance. The dipole is said to be oriented along the axis separating the monopoles. A dipole radiates sound symmetrically around its axis of orientation, with the greatest magnitude of pressure 
fluctuation along its axis, and zero pressure fluctuation about its equator. Quadrupole sources are not implemented in the present work, though it is noted that they are specific combinations of four monopoles [54].

The FW-H equation calculates the sound created by aerodynamic turbulence and surfaces in motion to be composed of a surface distribution of monopoles and dipoles, and a volume distribution of quadrupole sources. Ffowcs Williams and Hawkings showed that the thickness noise, which is the noise created by the volume displacement (or thickness) associated with a body, can be represented by distribution of monopole acoustic sources. Loading noise, which is caused by the aerodynamic force distribution over the surfaces of a body, can similarly be represented by a distribution of dipole acoustic sources. Compressibility effects contribute to the quadrupole noise, which is significantly less than the thickness and loading noise for flows in the subsonic regime $[24,31]$.

\section{$3.2 \quad$ Aeroacoustic methods}

The geometric representation of the problem is a simplification of the representation used for the aerodynamic methods, with the quadrilateral surface panels of the rotor blades treated as point sources located at the centroid of each panel. All other geometric features are ignored including the central tower, guy wires, ground, and any surrounding structures. This representation is used to obtain results which are dependent only on the flow conditions around the blades. In practice, geometric features such as the ground and surrounding structures may reflect and/or impede the acoustics. Also excluded from this analysis are the aeolian tones which the central tower and guy wires may produce due to the unsteady wake shedding from them.

The atmosphere is represented as a homogeneous medium with uniform pressure, 
density, and free-stream velocity. Many environmental factors are thereby neglected, including acoustic attenuation and inflow turbulence. The wake-induced turbulence is expected to contribute vortex sound to the overall acoustic signal in the wake regions but these effects are presently neglected due to the additional emphasis required to accurately model the problem's wake and the limited time frame of this work [23].

The unsteady inviscid panel method used to predict the gauge pressure and flow velocity over the surface of the rotor blades does not capture boundary layers. The lack of a turbulent boundary layer, combined with the laminar atmospheric inflow, will result in smaller broadband noise predictions. It is expected that the majority of the acoustic signal will be tonal in nature, at multiples of the blade passage frequency (BPF). The wake-blade interaction may contribute a broadband noise component [5].

\subsubsection{Advanced time approach}

For an acoustic simulation, there are two main time domains which are of concern: source time $\tau$, and observer time $t$. An acoustic emission released from the source at time $\tau$ and position $\mathbf{y}$ is received by an observer located at $\mathbf{x}$ at time $t$.

Due to Doppler effects and the variation in the acoustic radiation distance at any given source time step, a discretized source time domain does not correspond to an equally spaced discretization of the observer time domain [55]. As such, the observer time resolution is typically much greater than the source (aerodynamic) time resolution, which allows for acoustic signals with higher frequencies to be captured.

The acoustic simulation in the present work follows an advanced time approach such that each time step of the acoustic simulation corresponds to an aerodynamic simulation (source) time step.

The perturbation pressure at the observer position for each acoustic source is 
calculated using an acoustic analogy formulation. Following the advanced time approach, the time at which this perturbation pressure is experienced by the observer is [33]

$$
t_{\mathrm{adv}}=\tau_{e}+\frac{R}{c_{0}},
$$

where $\tau_{e}$ is the emission source time, $R$ is acoustic radiation distance at emission time $\tau_{e}$, and $c_{0}$ is the speed of sound in the undisturbed medium.

\subsubsection{Formulation $1 \mathrm{C}$}

Generally speaking, wind turbine rotors are moving acoustic sources in a moving medium. The moving medium is the wind which is assumed to be uniform over the entire spatial domain [33].

An extension to the classical FW-H equation and its Formulation 1A is presented by Najafi-Yazdi et al. [33] as Formulation 1C, which was derived using the convective wave equation to account for the moving medium effects that are neglected by other formulations of the FW-H equation. This work derives a form of Formulation 1C that is in a convenient form for application to a discretized non-penetrable geometry, and uses it to calculate the thickness and loading acoustic signals from VAWT rotors.

Najafi-Yazdi et al. introduce a number of terms and notations in their derivation of Formulation 1C. To aid reader comprehension, a brief overview of these terms and the unique aspects of the derivation are included here. Additional details on the derivation of Formulation 1C for discretized non-penetrable geometry experiencing inviscid flow conditions are included in Appendix B.1.

For simplicity, Formulation 1C is derived for the special case of having the medium move uniformly along only the 1-direction, (such that $\mathbf{U}_{\infty}=U_{\infty 1} \hat{\mathbf{e}}_{1}$ ) and all equations presented here follow that assumption. 
The notation for thickness sources is the scalar $Q_{n}$, and the notation for loading sources is the vector $L_{n_{i}}$. These terms are calculated at the source positions as

$$
\begin{gathered}
Q_{n}=\rho_{0}\left(v_{i}-U_{\infty i}\right) \hat{n}_{i} \\
L_{n_{i}}=p^{\prime} \hat{n}_{i}
\end{gathered}
$$

where $v_{n}$ is the component of velocity of the surface in the direction normal to the surface and $p^{\prime}$ is the perturbation pressure on the surface.

The acoustic radiation distance $R$ between a source at position $\mathbf{y}$ and an observer at $\mathbf{x}$ is calculated as [33]

$$
R=\frac{-M_{\infty}\left(x_{1}-y_{1}\right)+R^{*}}{\beta^{2}}
$$

where the quantity $R^{*}$ and the Prandtl-Glauert factor $\beta$ are calculated as [33]

$$
\begin{gathered}
R^{*}=\sqrt{\left(x_{1}-y_{1}\right)^{2}+\beta^{2}\left[\left(x_{2}-y_{2}\right)^{2}+\left(x_{3}-y_{3}\right)^{2}\right]}, \\
\beta=\sqrt{1-M_{\infty}^{2}} .
\end{gathered}
$$

The general expressions for the thickness and loading perturbation pressures are calculated in [33] as

$$
\begin{gathered}
4 \pi p_{T}^{\prime}\left(\mathbf{x}, t_{\mathrm{adv}}\right)=\left[\frac{\partial}{\partial t}+U_{\infty} \frac{\partial}{\partial x_{1}}\right] \int_{-\infty}^{t} \int_{\mathbb{R}^{3}} Q_{j} n_{j} \delta(f) \frac{\delta(g)}{R^{*}} \mathrm{~d}^{3} \mathbf{y} \mathrm{d} \tau \\
4 \pi p_{L}^{\prime}\left(\mathbf{x}, t_{\mathrm{adv}}\right)=-\frac{\partial}{\partial x_{i}} \int_{-\infty}^{t} \int_{\mathbb{R}^{3}}\left[L_{i j} n_{j} \delta(f)\right] \frac{\delta(g)}{R^{*}} \mathrm{~d}^{3} \mathbf{y} \mathrm{d} \tau
\end{gathered}
$$


where $\delta$ is the Kronecker delta function and the parameter $g$ is defined as [33]

$$
g=\tau-t+\frac{R}{c_{0}}
$$

Then, the far-field perturbation pressures are

$$
\begin{aligned}
4 \pi p_{T}^{\prime}\left(\mathbf{x}, t_{\text {adv }}\right)=\sum_{k=1}^{N} & {\left[\left(\frac{\dot{Q}_{n}}{R^{*}\left(1-M_{R}\right)^{2}}-\frac{\partial R^{*}}{\partial \tau} \frac{Q_{n}}{R^{* 2}\left(1-M_{R}\right)^{2}}+\frac{Q_{n}}{R^{*}\left(1-M_{R}\right)^{3}} \frac{\partial M_{R}}{\partial \tau}\right)\right.} \\
& -M_{\infty}\left(\frac{\dot{\tilde{R}}_{1} Q_{n}+\tilde{R}_{1} \dot{Q}_{n}}{R^{*}\left(1-M_{R}\right)^{2}}-\frac{\partial R^{*}}{\partial \tau} \frac{\tilde{R}_{1} Q_{n}}{R^{* 2}\left(1-M_{R}\right)^{2}}+\frac{\partial M_{R}}{\partial \tau} \frac{\tilde{R}_{1} Q_{n}}{R^{*}\left(1-M_{R}\right)^{3}}\right) \\
& \left.-U_{\infty}\left(\frac{\tilde{R}_{1}^{*} Q_{n}}{R^{* 2}\left(1-M_{R}\right)}\right)\right]_{k} S_{k}, \\
4 \pi p_{L}^{\prime}\left(\mathbf{x}, t_{\text {adv }}\right)=\sum_{k=1}^{N} & {\left[\frac{1}{c_{0}}\left(\frac{\dot{L}_{n_{i}} \tilde{R}_{i}+L_{n i} \dot{\tilde{R}}_{i}}{R^{*}\left(1-M_{R}\right)^{2}}-\frac{\partial R^{*}}{\partial \tau} \frac{L_{n i} \tilde{R}_{i}}{R^{* 2}\left(1-M_{R}\right)^{2}}+\frac{\partial M_{R}}{\partial \tau} \frac{L_{n i} \tilde{R}_{i}}{R^{*}\left(1-M_{R}\right)^{3}}\right)\right.} \\
& \left.+\frac{L_{n i} \tilde{R}_{i}^{*}}{R^{* 2}\left(1-M_{R}\right)}\right]_{k} S_{k},
\end{aligned}
$$

where $M_{R}$ is the component of the Mach number of the surface in the radiation direction

$$
M_{R}=\frac{1}{c_{0}} v_{i} \tilde{R}_{i}
$$

and the scalar gradients $\tilde{R}_{i}$ and $\tilde{R}_{i}^{*}$ are [33]

$$
\begin{aligned}
& \tilde{R}_{i}^{*}=\frac{\partial}{\partial x_{i}} R^{*} \Rightarrow \tilde{R}_{1}^{*}=\frac{x_{1}-y_{1}}{R^{*}}, \tilde{R}_{2}^{*}=\beta^{2} \frac{x_{2}-y_{2}}{R^{*}}, \tilde{R}_{3}^{*}=\beta^{2} \frac{x_{3}-y_{3}}{R^{*}} \\
& \tilde{R}_{i}=\frac{\partial}{\partial x_{i}} R \Rightarrow \tilde{R}_{1}=\frac{1}{\beta^{2}}\left(-M_{\infty}+\tilde{R}_{1}^{*}\right), \tilde{R}_{2}=\frac{x_{2}-y_{2}}{R^{*}}, \tilde{R}_{3}=\frac{x_{3}-y_{3}}{R^{*}}
\end{aligned}
$$


For the special case of zero mean flow velocity, thickness and loading equations for the non-penetrable form of Formulation $1 \mathrm{C}$ can be shown to be equivalent to the thickness and loading equations for non-penetrable form of Formulation 1A presented by Farassat in [27]. For mean flows with a low Mach number $\left(M_{\infty}<0.1\right)$, Formulations 1A and 1C produce nearly identical results.

The terms in Eq. (3.10) and (3.11) with $R^{* 2}$ in the denominator (called the "nearfield" terms) diminish quicker with increasing acoustic radiation distance than terms with $R^{*}$ in the denominator (called the "far-field" terms).

It is important to note that the perturbation pressures obtained using Eq. (3.10) and (3.11) are not strictly equivalent to the corresponding thickness and loading acoustic pressures. Each perturbation pressure $p^{\prime}$ can be considered to have a mean (time-averaged) component $\overline{p^{\prime}}$ and an fluctuating or oscillatory component $\widetilde{p^{\prime}}$. The fluctuating component is the acoustic pressure and is calculated as

$$
\widetilde{p^{\prime}}(\mathbf{x}, t)=p^{\prime}(\mathbf{x}, t)-\overline{p^{\prime}}(\mathbf{x}) .
$$

The total perturbation and acoustic pressures in the present method are the sum of the respective thickness and loading pressures. Unless otherwise specified, this work considers the total pressures.

The magnitude of acoustic pressure is often expressed as a RMS average of acoustic pressure and as a sound pressure level (SPL) in decibels. For any given observer, these quantities are respectively calculated as [54]

$$
\begin{gathered}
{\widetilde{p^{\prime}}}_{\text {rms }}=\sqrt{\overline{\left(\widetilde{p^{\prime}}\right)^{2}},} \\
S P L=20 \log _{10}\left(\frac{\widetilde{p_{\text {rms }}^{\prime}}}{p_{\text {ref }}}\right),
\end{gathered}
$$


where $p_{\text {ref }}=2 \times 10^{-5} \mathrm{~Pa}$ and $S P L$ is expressed in terms of decibels $(\mathrm{dB})$. Frequently, the notation "dB re $2 \times 10^{-5} \mathrm{~Pa}$ " is used to indicate that the decibels are determined relative to the pressure $2 \times 10^{-5} \mathrm{~Pa}$.

\subsubsection{Aeroacoustic component implementation}

The aeroacoustic component is implemented as a program written in FORTRAN 90 with OpenMP, separate from the aerodynamic component. The aeroacoustic program reads input text files for setup parameters (including observer positions and time resolution) and reads the output from the aerodynamic program to obtain information about the aeroacoustic sources.

While the aeroacoustics may be solved in parallel with the aerodynamic simulation, implementing the aeroacoustic component as a separate post-processing program enables the user to perform acoustic simulations at different observer positions and time resolutions without needing to re-run the time-consuming aerodynamic simulation.

Beyond the values which the aeroacoustic program obtains from the output of the aerodynamic program, the position and time resolution for all observers are specified by the user. Many observer positions may be considered during a single aeroacoustic simulation, allowing for entire fields of perturbation pressures to be solved simultaneously.

The required observer time discretization is not equivalent to the source (aerodynamic) time discretization. Each aeroacoustic source may have a unique acoustic radiation distance to any given observer due to differences in geometric distance as well as Doppler effects [55], which requires the observer time discretization to have a greater resolution. To resolve acoustic frequencies of up to $f_{\max }$, a simulation must use a time increment of at most the reciprocal of one-half of $f_{\max }$ based on the concept 
of the Nyquist frequency. To ensure sufficient resolution of perturbation pressures up to a frequency of $f_{\max }$, the present work selects the observer time increment $\Delta t$ to be ten times smaller than the Nyquist frequency requires, such that

$$
\Delta t \leq \frac{1}{10\left(0.5 f_{\max }\right)} .
$$

The aeroacoustic program first reads in the setup parameters, initializes, then proceeds through the source time steps (the output time steps from the aerodynamic simulation) calculating for each time step the thickness and loading perturbation pressures using Eq. (3.10) and (3.11), and the corresponding time at which they are experienced for each observer using Eq. (3.1).

For any given acoustic source, the time at which its perturbation pressure is experienced by the observer will likely not be exactly on an interval of discretized observer time. Additionally, a physical acoustic source will exist continuously in time, rather than solely on intervals of discretized source time as in the aeroacoustic simulation. These issues are addressed by linearly interpolating for the values of perturbation pressure on the interval between the exact times of observed perturbation pressures.

Figure 3.1 contains a schematic of the source and observer time frames for two acoustic sources which are at separate positions and therefore unique radiation distances. The contribution of each acoustic source is represented as half-circles at every interval of source time and relates to a perturbation pressure at a future observer time, represented by arrows with half-white tips. The offset between source time and observer time, represented by the dashed lines connecting the time axes, is dependent on the radiation distance and speed of sound, as described by Eq. (3.1). The arrows with solid triangular tips along the observer time axis are the interpolated values of perturbation pressure at every increment of observer time for the acoustic source. The 


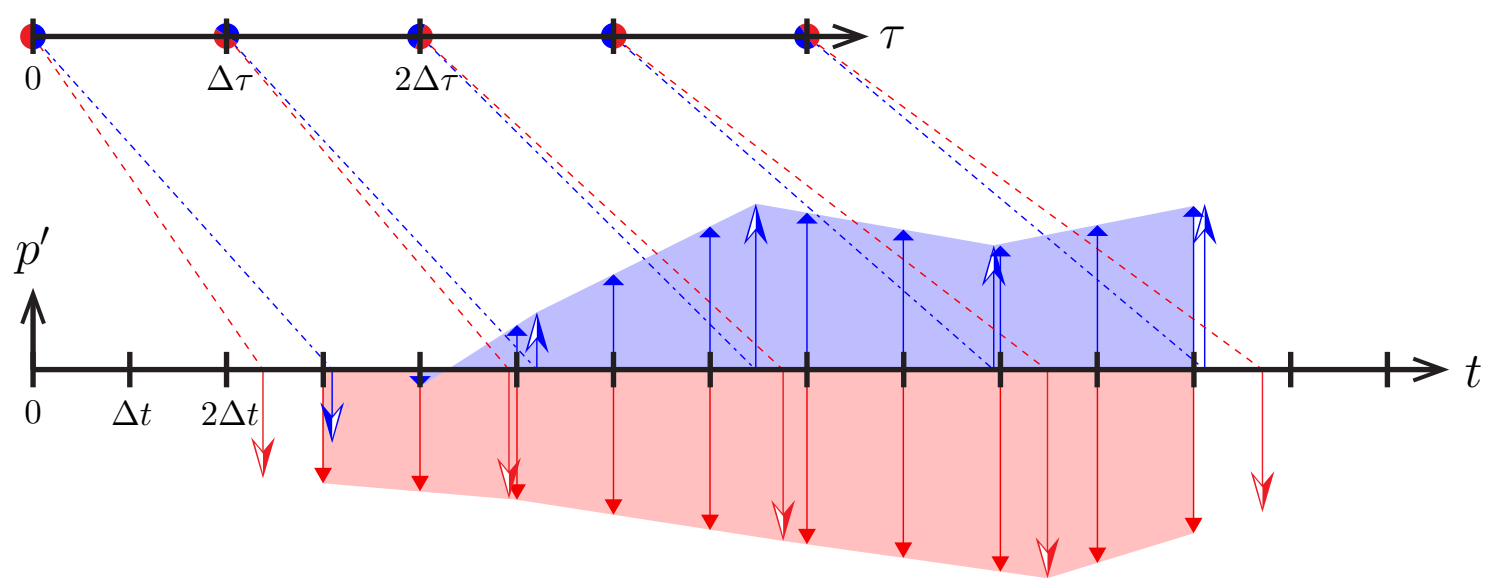

Figure 3.1: Illustration of the relationship between source and observer time frames for two moving acoustic sources (shown as red and blue) at separate positions

shaded regions represent the program's interpretation of the time-history of observed perturbation pressure.

After all source time steps have been processed, the time-history of the perturbation pressures are exported to files for post-processing. The time histories of the total thickness and loading perturbation pressures are found from the sum of the perturbation pressure time-histories of each thickness-contributing and loading-contributing source, respectively.

The perturbation pressures may be exported as either the total thickness and loading perturbation pressure histories for each observer, or as the thickness and loading histories for each observer due to each acoustic source. The latter option allows for the contribution from each acoustic source, or panel from the discretized geometry, to be considered separately, and may be used to provide insight into the aeroacoustic impact of localized regions of the VAWT rotor blades. 


\subsection{Aeroacoustic model verification}

To verify the implementation of the aeroacoustic component, the results from three fundamental test cases were considered:

(I) The acoustic radiation pattern due to a stationary monopole;

(II) The acoustic radiation pattern due to a stationary dipole; and,

(III) The perturbation pressure time-history at a single observer position due to a pulsating, rotating monopole.

From Najafi-Yazdi et al. [33], the perturbation pressure due to a pulsating monopole can be found from velocity potential as

$$
p^{\prime}(\mathbf{x}, t)=-\rho_{0}\left[\frac{\partial}{\partial t}+U_{\infty} \frac{\partial}{\partial x_{1}}\right] \operatorname{Re}(\Phi),
$$

where $\mathbf{U}_{\infty}=U_{\infty} \hat{\mathbf{e}}_{\mathbf{1}}$ as before, $\operatorname{Re}(\Phi)$ is the real component of velocity potential, and the velocity potential of a monopole is

$$
\Phi_{\mathrm{m}}(\mathbf{x}, t)=\frac{A_{\mathrm{m}}}{4 \pi R^{*}} \exp \left[i \omega\left(t-\frac{R}{c_{0}}\right)\right]
$$

with $\omega$ as the pulsation frequency of the acoustic source and $A_{\mathrm{m}}$ as the potential semi-amplitude of the of the monopole (in $\mathrm{m}^{3} / \mathrm{s}$ ).

Case (I) considers the RMS of perturbation pressure experienced by observers radially around a source at a distance of 30 metres. The potential solution considers the source to have a semi-amplitude of $1 \mathrm{~m}^{3} / \mathrm{s}$ and a pulsation frequency of 5 Hertz. To replicate the monopole using the present method's formulation, a single point source is considered with an effective area of $S=1 \mathrm{~m}^{2}$, a normal velocity component pulsating with a semi-amplitude of $1 \mathrm{~m} / \mathrm{s}$ at a rate of 5 Hertz, and a reference density 


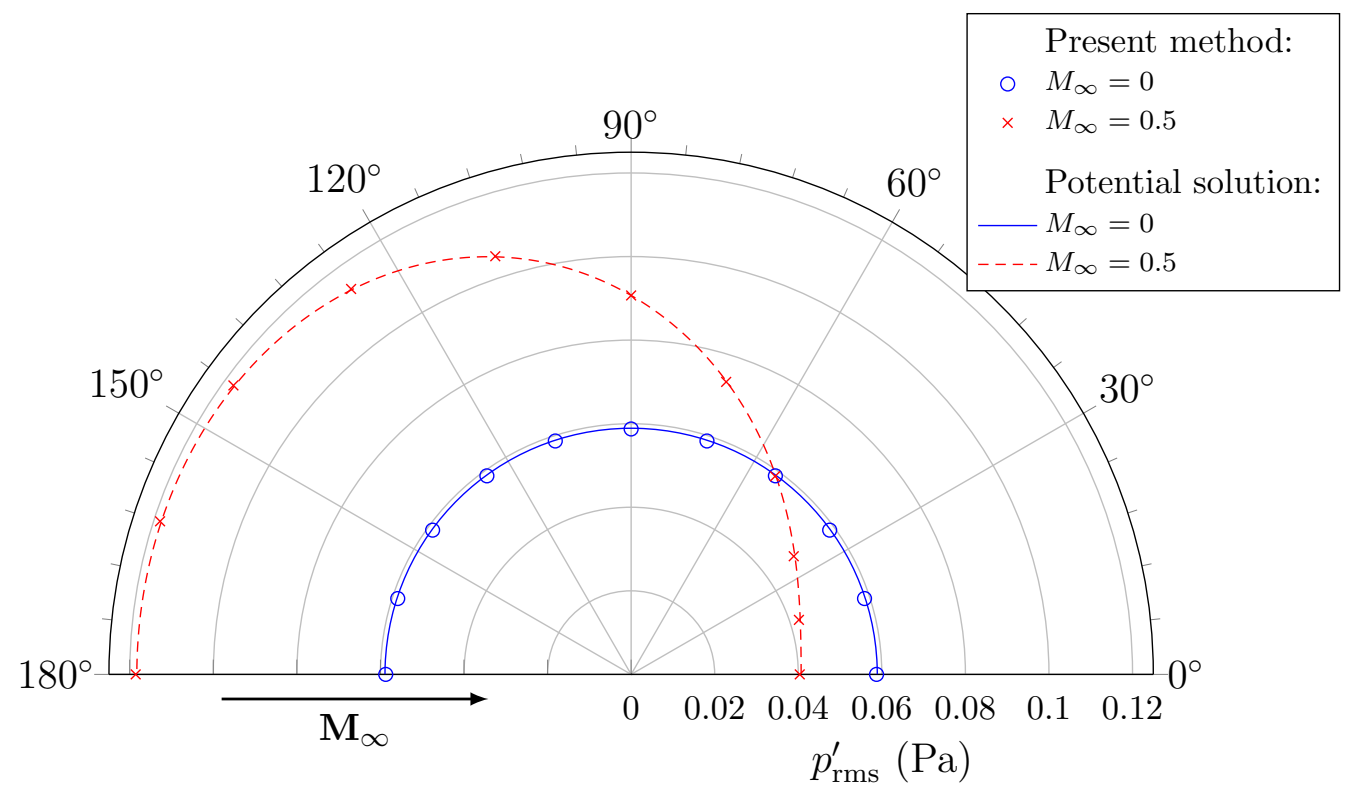

Figure 3.2: Monopole perturbation pressure radiation pattern at an observer distance of 30 metres

of $\rho_{0}=1 \mathrm{~kg} / \mathrm{m}^{3}$. The results for this case under mean flow conditions of $M_{\infty}=0$ and $M_{\infty}=0.5$ are shown in Fig. 3.2, and show excellent agreement.

The dipole in Case (II) is considered to be oriented in the 2-direction, perpendicular to the free-stream flow. Similar to the monopole, the strength of the dipole, $L_{n i}$, pulsates with a semi-amplitude of 1 Pascal at a frequency of 5 Hertz. Fig. 3.3 shows the radiation pattern obtained for this dipole using the present method for observers at a distance of 30 metres. It can be seen that the directivity of the RMS of perturbation pressure follows a trend expected of a dipole, with the peak value (along the $90^{\circ}$ azimuth direction) approximately equal to the RMS of perturbation pressure for the monopole at $M_{\infty}=0$ divided by the speed of sound $\left(c_{0}=340 \mathrm{~m} / \mathrm{s}\right)$, as expected from inspection of Eq. (3.10) and (3.11).

Case (III) considers the perturbation pressure induced by a pulsating monopole, with semi-amplitude and pulsation frequency equal to the respective values from Case (I), rotating about the 3 -direction at a distance of $0.7 \mathrm{~m}$ and an angular frequency 


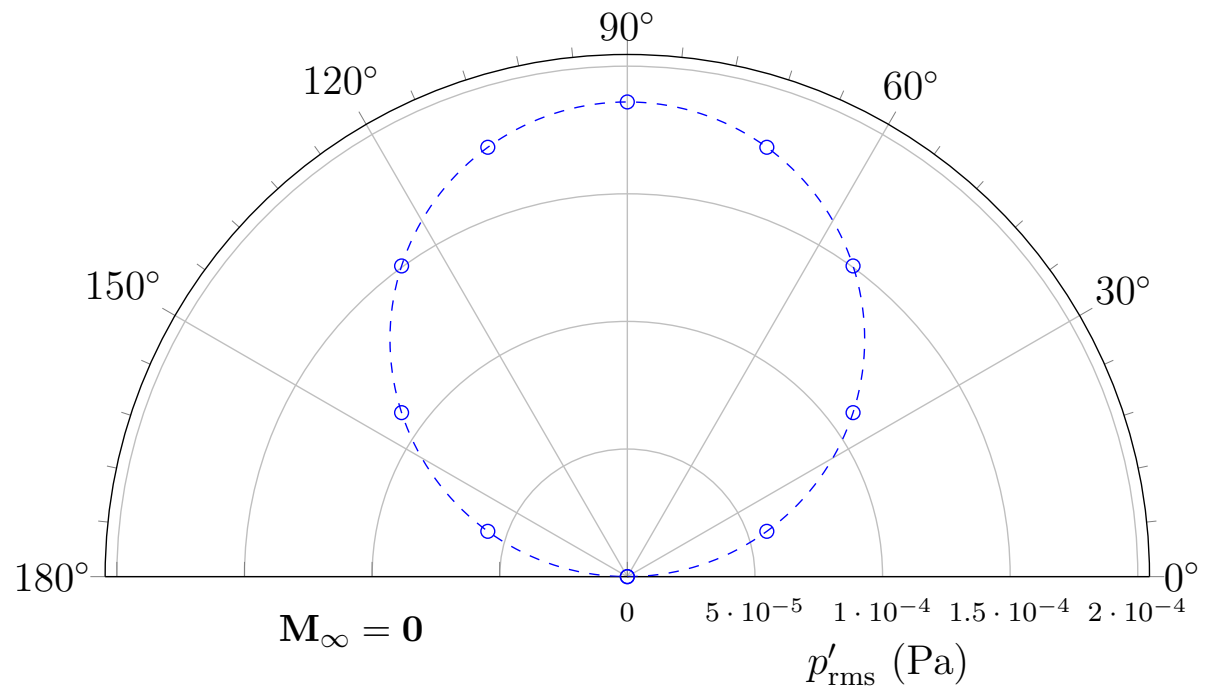

Figure 3.3: Dipole perturbation pressure radiation pattern at an observer distance of 30 metres for a dipole oriented in the $90^{\circ}$ direction

of $1 \mathrm{~Hz}$ at an observer position $2 \mathrm{~m}$ down-stream of the centre of the monopole's rotation, as shown in Fig. 3.4a. The time-histories of perturbation pressure in Fig. 3.4b once again show excellent agreement between the present implementation of Formulation $1 \mathrm{C}$ and the results from the potential solution. 


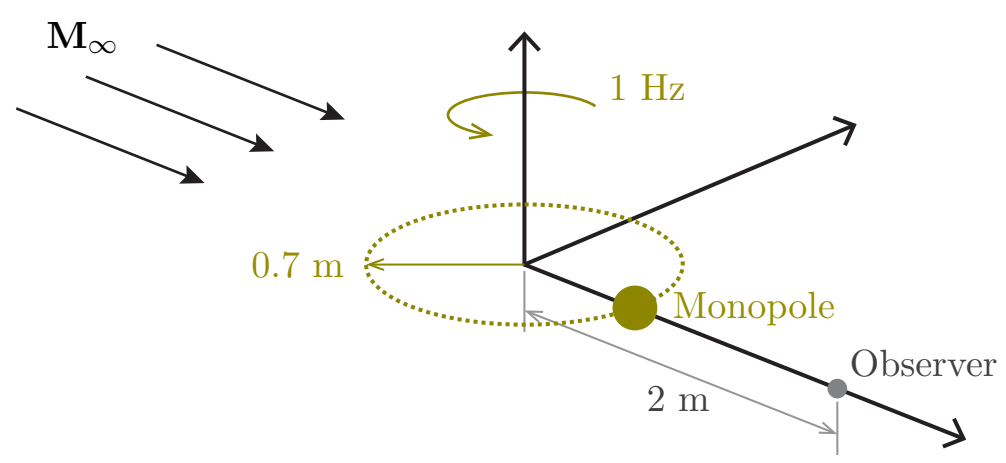

(a) Diagram of the rotating monopole (based on [33])

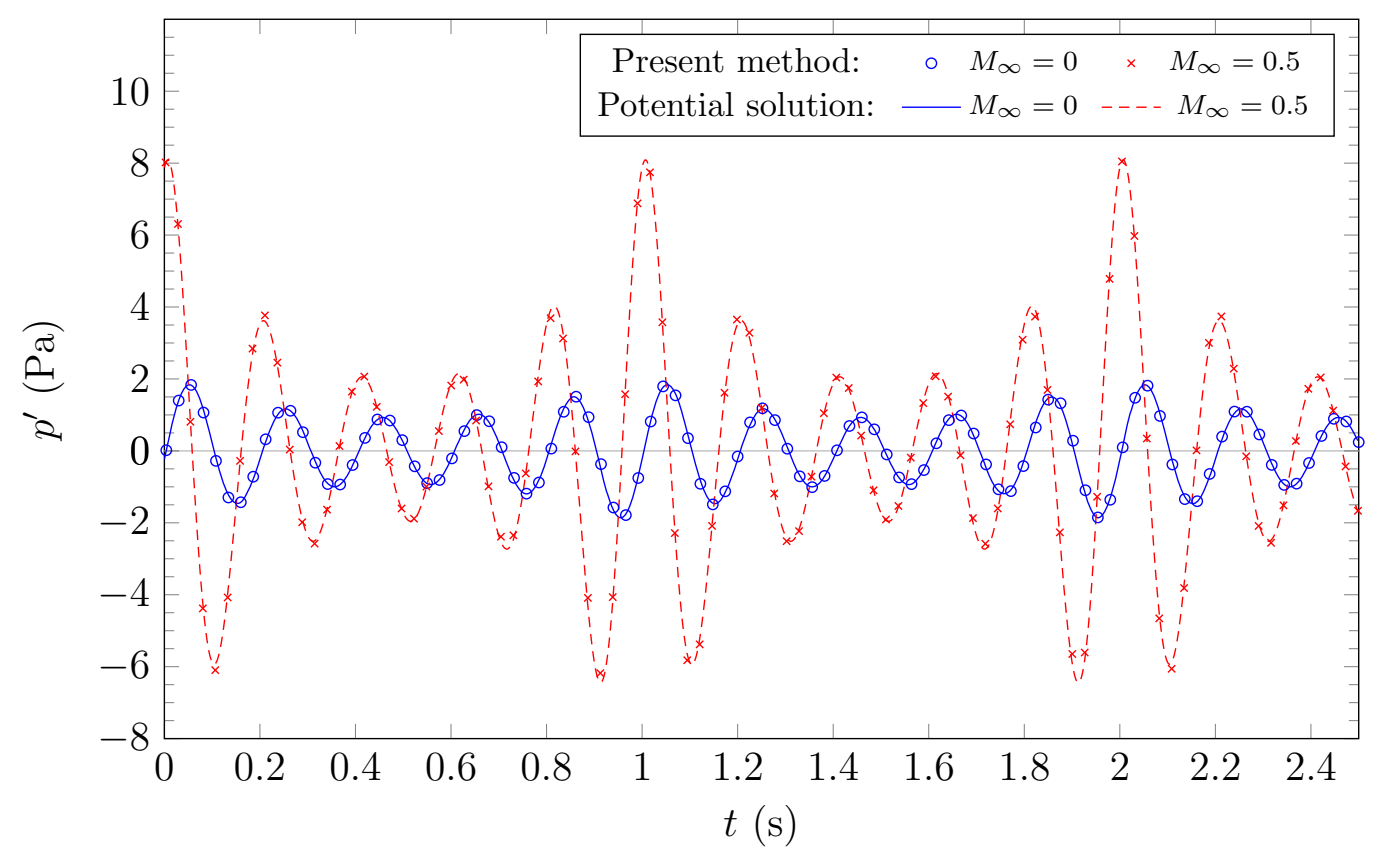

(b) Perturbation pressure history of a rotating monopole with pulsation frequency of $5 \mathrm{~Hz}$ in a free-stream flow $M_{\infty}$ observed $2 \mathrm{~m}$ downwind of the centre of rotation

Figure 3.4: A schematic of the rotating monopole and observer positions and the observed perturbation pressure histories 


\section{Chapter 4}

\section{Results and Discussions}

This chapter presents and discusses results of the present method's approach to modelling VAWT aerodynamics and aeroacoustics.

Spatial and temporal convergence analyses are first presented to determine a suitable geometric discretization and aerodynamic simulation time-step size. Verification of the aerodynamic results for VAWTs is made by comparing non-dimensional tangential and normal forces obtained using the present method with those obtained using a semi-empirical method. Aeroacoustic simulation results for a VAWT are compared with experimental results.

After the discrepancies between the present method and other published aerodynamic and aeroacoustic results are characterized, the aerodynamics and aeroacoustics of VAWTs are studied with investigations into their dependence on various VAWT rotor parameters.

All results assumed an atmospheric density of $\rho_{0}=1.205 \mathrm{~kg} / \mathrm{m}^{3}$ and a speed of sound of $c_{0}=340 \mathrm{~m} / \mathrm{s}$. All rotor blade geometries are such that the quarter-chord point of the airfoils are placed on the circle of rotation at the specified rotor radius and have zero angle of incidence relative to the path of motion, as shown in Fig. 1.2. 


\subsection{Convergence Analyses}

Before obtaining results, it is important to know whether the discretization parameters used will have a great enough resolution to be able to obtain reliable results. A good understanding of the convergence characteristics of the discretization may help avoid the large computational time associated excessively fine discretizations.

Spatial and temporal convergence have been performed for the Sandia 17-m VAWT, which is described by the parameters in Table 4.1 and shown in Fig. 4.1.

\begin{tabular}{|l|c|}
\hline Blade shape & Troposkein \\
\hline Airfoil & NACA 0015 \\
\hline Rotor diameter, $d_{\mathrm{T}}$ & $17.0 \mathrm{~m}$ \\
\hline Diameter of central tower & $0.762 \mathrm{~m}$ \\
\hline Rotor height, $h_{\mathrm{T}}$ & $25.15 \mathrm{~m}$ \\
\hline Rotor elevation above ground & $2.7 \mathrm{~m}$ \\
\hline Chord length, $c_{\mathrm{T}}$ & $0.610 \mathrm{~m}$ \\
\hline Rotational speed, $\Omega_{\mathrm{T}}$ & $48 \mathrm{RPM}$ \\
\hline Wind speed, $U_{\infty}$ & $8.0 \mathrm{~m} / \mathrm{s}$ \\
\hline Tip speed ratio, $\lambda_{\mathrm{T}}$ & 5.34 \\
\hline
\end{tabular}

Table 4.1: Parameters of the Sandia 17-m VAWT, [56]

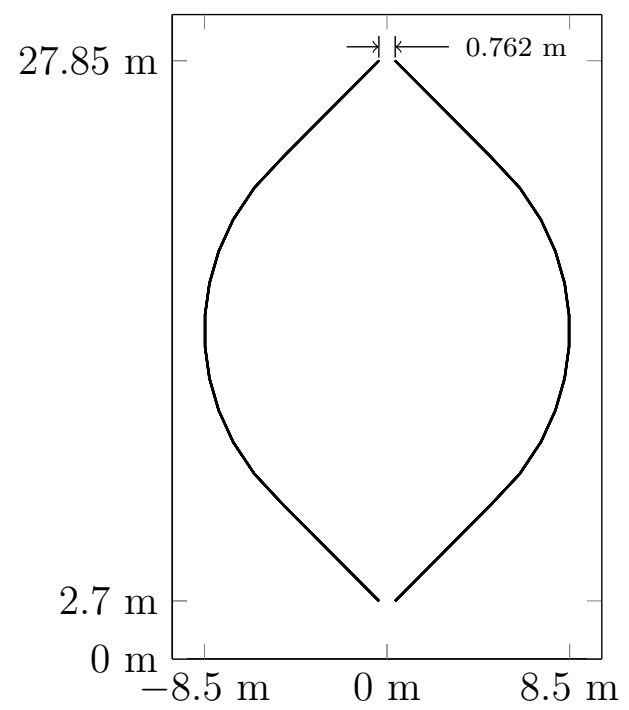

Figure 4.1: Profile of the Sandia 17-m rotor, based on [57]

\subsubsection{Spatial Convergence}

The aeroacoustic results do not require as fine a spatial resolution as the aerodynamic results for the typical distances an observer would be away from an operating VAWT. The aerodynamic results are therefore monitored for spatial convergence.

Spatial convergence analysis was performed by monitoring the non-dimensional tangential and normal forces at the equator of one blade of a VAWT over the azimuth of a single revolution at periodic steady-state. Simulations with independently varying numbers of rotor blade span-wise sections $\left(N_{h}\right)$, and line segments which outline the 
airfoil of the rotor blades, $\left(N_{a}\right)$, were performed. While varying the number of blade span-wise sections, 20 line segments were used to outline the airfoil profile. While varying the number of line segments for the airfoil profile, 17 blade span-wise sections were used.

The results of these series of simulations, as seen Fig. 4.2 and Fig. 4.3, show good convergence in the span-wise discretization by 17 span-wise sections, and acceptable convergence by 50 line segments over the chord. A further increase in these values does not justify the accompanying increase in computational cost.

\subsubsection{Temporal Convergence}

The temporal resolution required for aeroacoustic convergence is expected to be greater than the resolution required for aerodynamic convergence. Hence, only the aeroacoustic results are analyzed for temporal convergence.

The root-mean-square and the power spectral density (PSD) of total acoustic pressure at an observer position $3 d_{\mathrm{T}}$ upwind and level with the equator of the Sandia 17-m VAWT are considered for temporal convergence.

Figure 4.4 shows convergence of the acoustic pressure's RMS value by an azimuth increment of $4.5^{\circ}$ (80 increments per revolution), which should therefore be sufficient for estimating the SPL. Figure 4.5 shows that an azimuth increment of $4.5^{\circ}$ (corresponding to a frequency of $100 \mathrm{~Hz}$ ) sufficiently resolves the PSD up to a frequency of $6.4 \mathrm{~Hz}$, the equivalent to four times the BPF.

Based on the trend for decreasing azimuth increment size in Fig. 4.5, it is expected that a smaller increment of azimuth angle will capture acoustics contributions at higher frequencies. However, doing so greatly increases the computational time required to achieve a steady state, and is counter-productive to the goal of having a computational tool which will provide sufficiently accurate results in minimal time. 

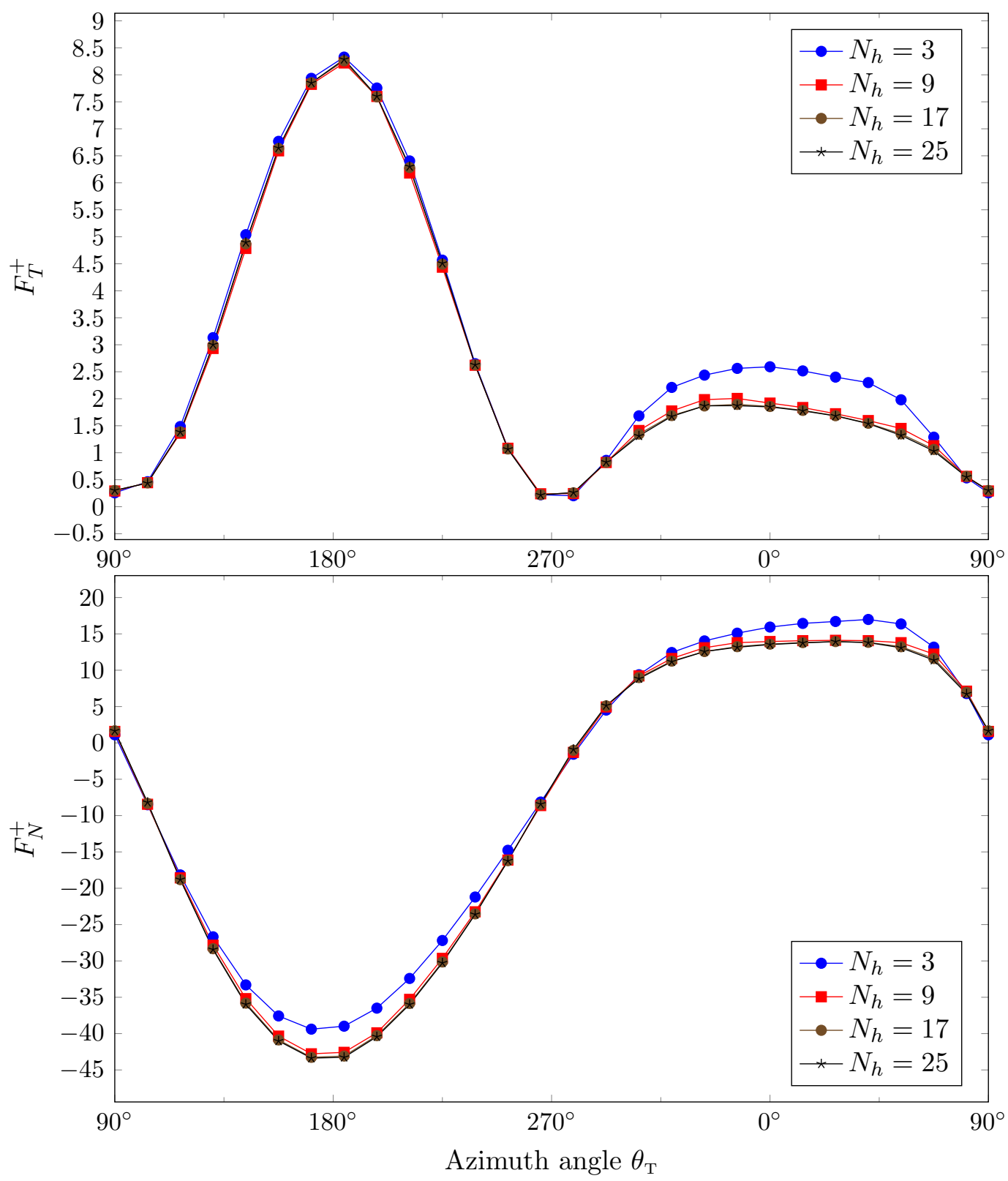

Figure 4.2: Convergence of rotor span-wise discretization using steady-state non-dimensional forces $F_{T}^{+}$and $F_{N}^{+}$at the equator 

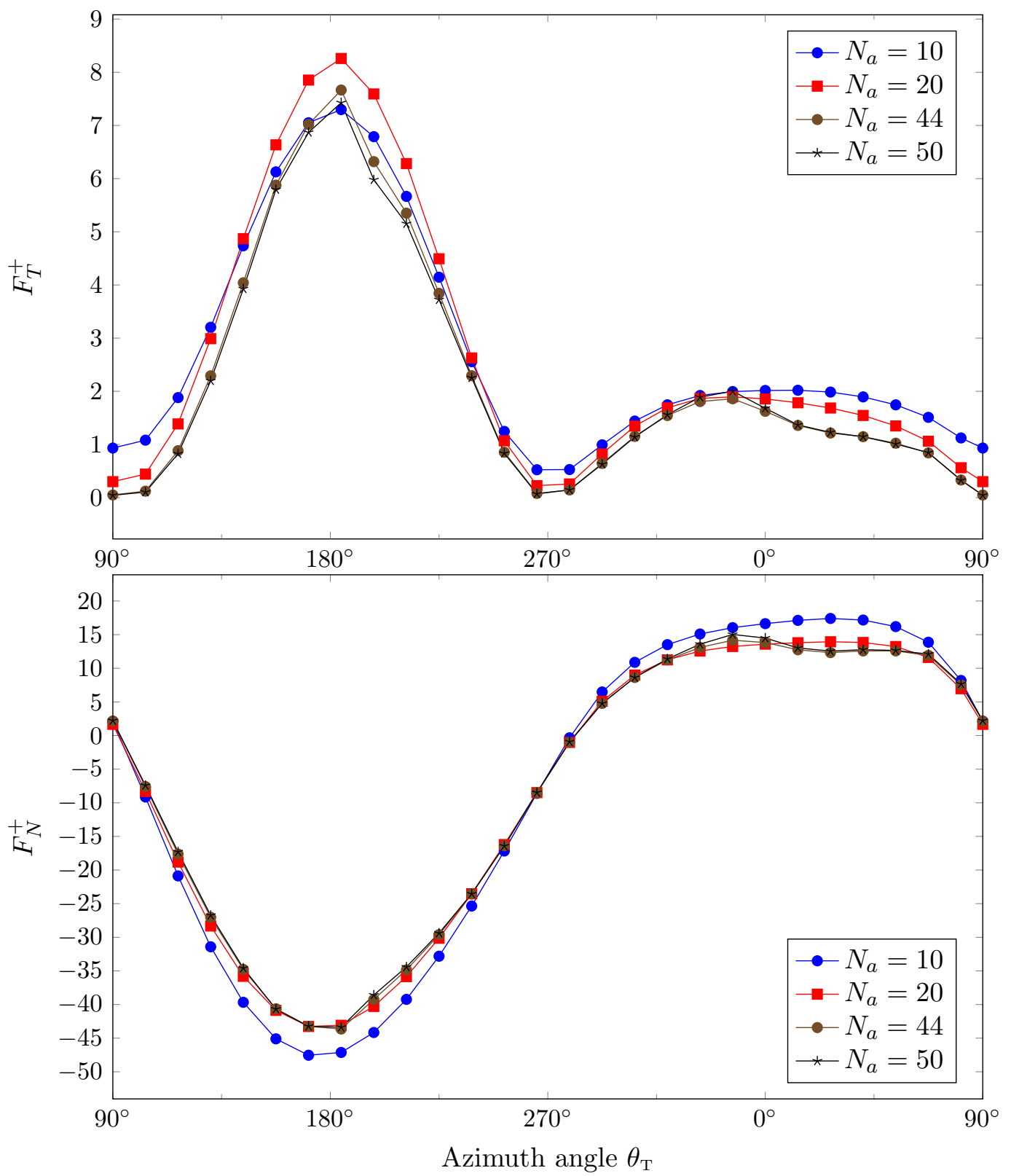

Figure 4.3: Convergence of rotor airfoil discretization using steady-state non-dimensional forces $F_{T}^{+}$and $F_{N}^{+}$at the equator 


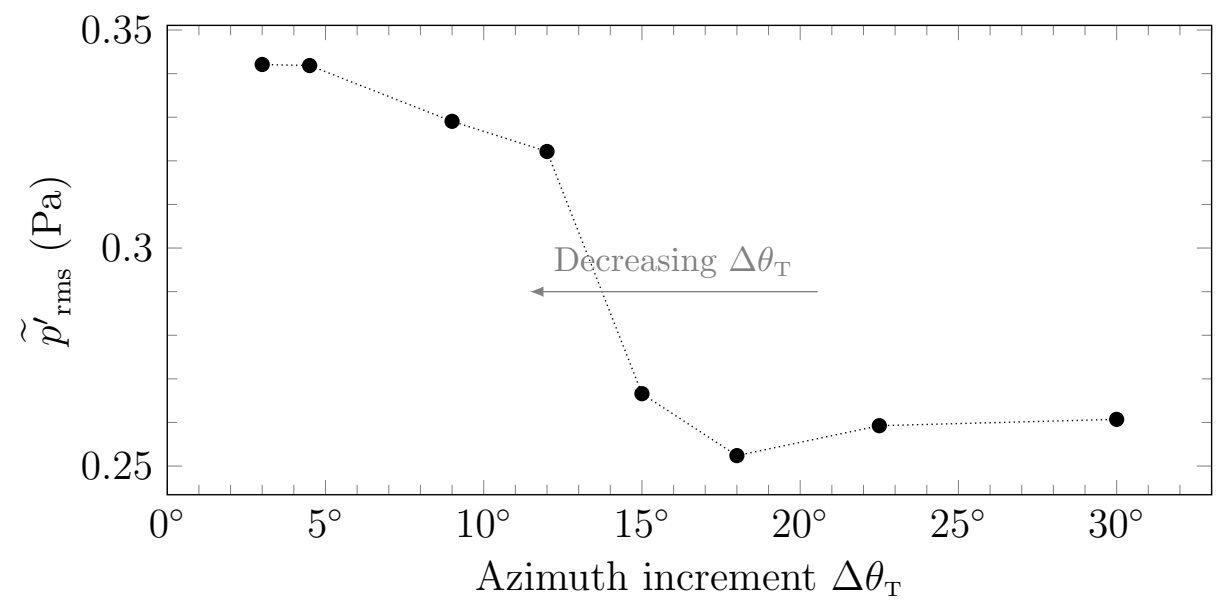

Figure 4.4: Convergence of the RMS of acoustic pressure for decreasing increment of azimuth angle

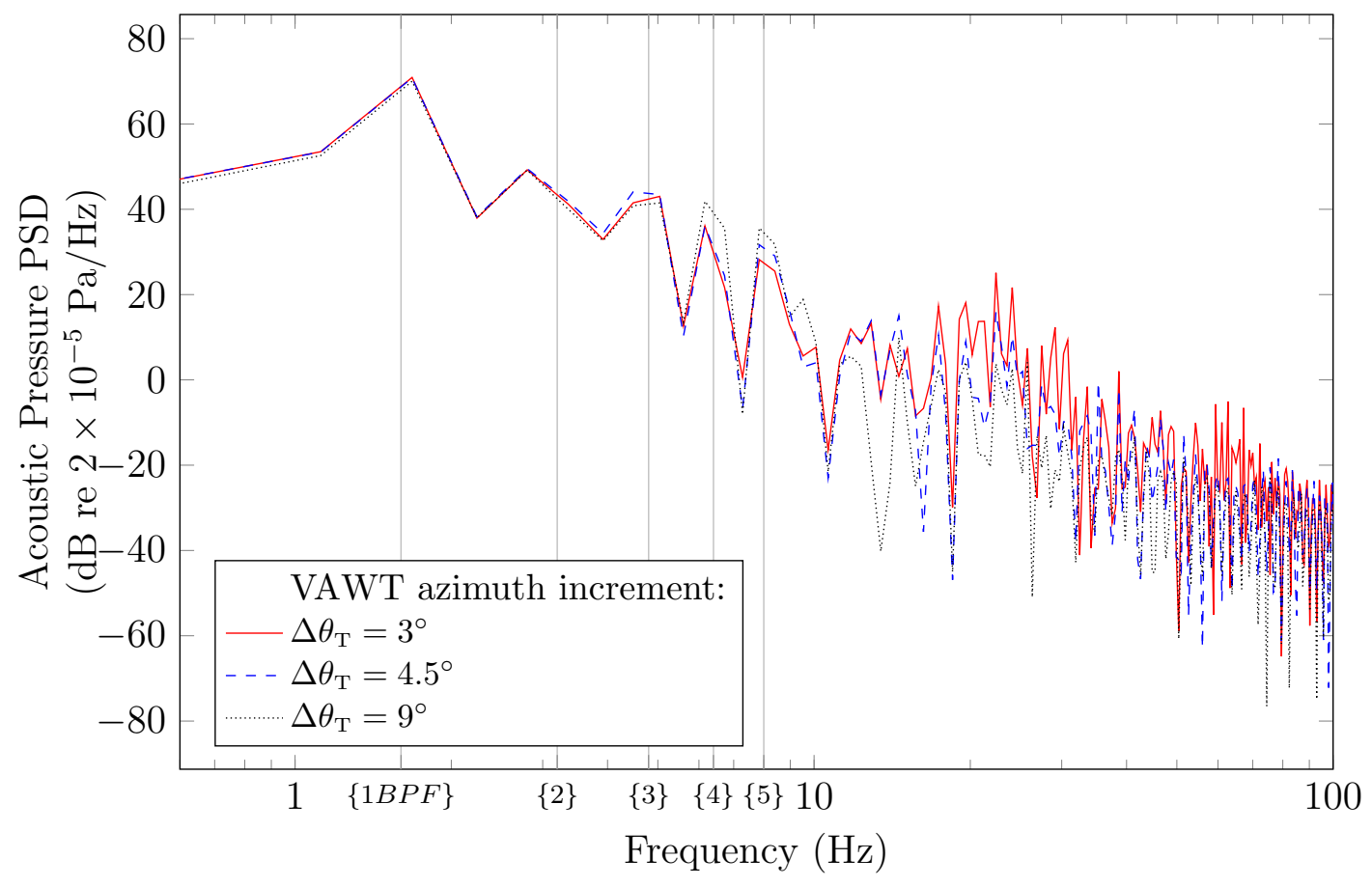

Figure 4.5: Acoustic pressure PSD for decreasing azimuth increment size 
Based on these results for spatial and temporal convergence, all VAWT aerodynamic investigations have been performed using a span-wise discretization of 17 , an airfoil discretization of 50 line segments, and an azimuth increment of $4.5^{\circ}$. With this discretization, an aerodynamic simulation with seven revolutions of a two-bladed rotor requires approximately two hours to run on a computer with an Intel $^{\mathrm{TM}} \mathrm{Core}^{\circledR}$ i7-3610QM CPU operating at 2.30 GHz with eight threads on four cores. The aeroacoustic simulation is significantly faster, and can generate results from such an aerodynamic simulation for thousands of observer positions in several minutes.

\subsection{Verification and Validation for VAWTs}

Simulating VAWT rotors involves complex unsteady aerodynamics with significant wake interactions producing highly variable acoustic sources. The aerodynamic and aeroacoustic results produced using the present work's method have been verified and validated for simple test cases in Sec. 2.3 and 3.3, and are compared to available results in this section.

Limited interest in VAWTs relative to their HAWT-counterparts for many years has led to only a small amount of published data presently available for the aerodynamics and especially the acoustics of VAWTs. As such, the aerodynamics are compared to results produced by "FEM-Vort", which is a code developed by Fereidooni [6], and the experimental acoustic results published by Kelley et al. [37].

\subsubsection{Aerodynamic Verification}

Fereidooni's program, FEM-Vort, is a semi-empirical aeroelastic code which represents the blades as a series of bound vorticities along the span and the wake as a freely-convecting cluster of vortex filaments [6]. Lift and drag data along the span 
of the blades are determined by interpolating experimental lift- and drag-coefficient curves for the airfoil at specific angles of attack and Reynolds numbers experienced along the blades.

Results obtained using the present method and the results published in [6] are compared in Fig. 4.7 using the development of the non-dimensional tangential and normal forces at the equator of a single rotor blade from the turbine described in Table 4.2 and shown in Fig. 4.6. As described in Sec. 1.3.1, a rotor blade at an azimuth angle of $0^{\circ}$ is at a position immediately downwind of the vertical axis, and immediately upwind position corresponds to an azimuth angle of $180^{\circ}$.

\begin{tabular}{|l|c|}
\hline Blade shape & Troposkein \\
\hline Airfoil & NACA 0012 \\
\hline Rotor diameter, $d_{\mathrm{T}}$ & $2 \mathrm{~m}$ \\
\hline Rotor height, $h_{\mathrm{T}}$ & $2 \mathrm{~m}$ \\
\hline Chord length, $c_{\mathrm{T}}$ & $0.135 \mathrm{~m}$ \\
\hline Rotational speed, $\Omega_{\mathrm{T}}$ & $45 \mathrm{rad} / \mathrm{s}$ \\
\hline Wind speed, $U_{\infty}$ & $9 \mathrm{~m} / \mathrm{s}$ \\
\hline Tip speed ratio, $\lambda_{\mathrm{T}}$ & 5.0 \\
\hline
\end{tabular}

Table 4.2: Parameters of the aerodynamic verification turbine

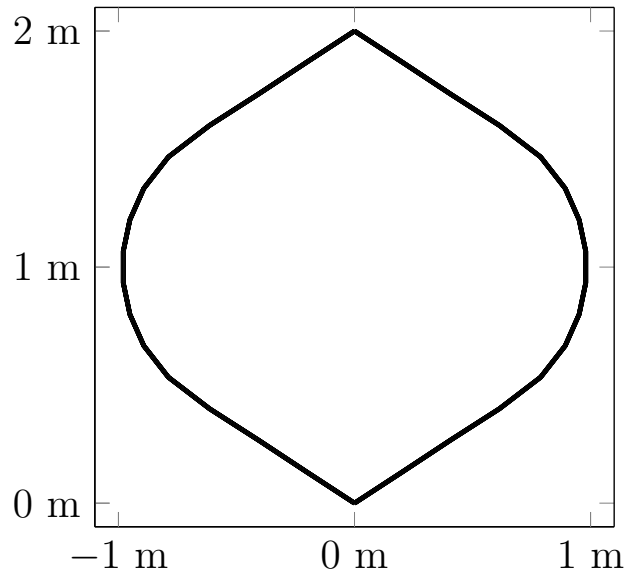

Figure 4.6: Profile of the aerodynamic verification rotor

The $F_{T}^{+}$peaks shown in Fig. 4.7 at upwind azimuth positions are nearly twice as large for the present method as the peaks predicted by FEM-Vort, while the values are in good agreement at downwind azimuth positions. This discrepancy in $F_{T}^{+}$at the upwind azimuth angles may be due to the present method not capturing the viscous drag effects on the blades, which act almost entirely in the tangential direction as seen in Fig. 1.2, and the presence of virtual camber and virtual angle of incidence as described in Sec. 1.3.1 which is not addressed by FEM-Vort. 

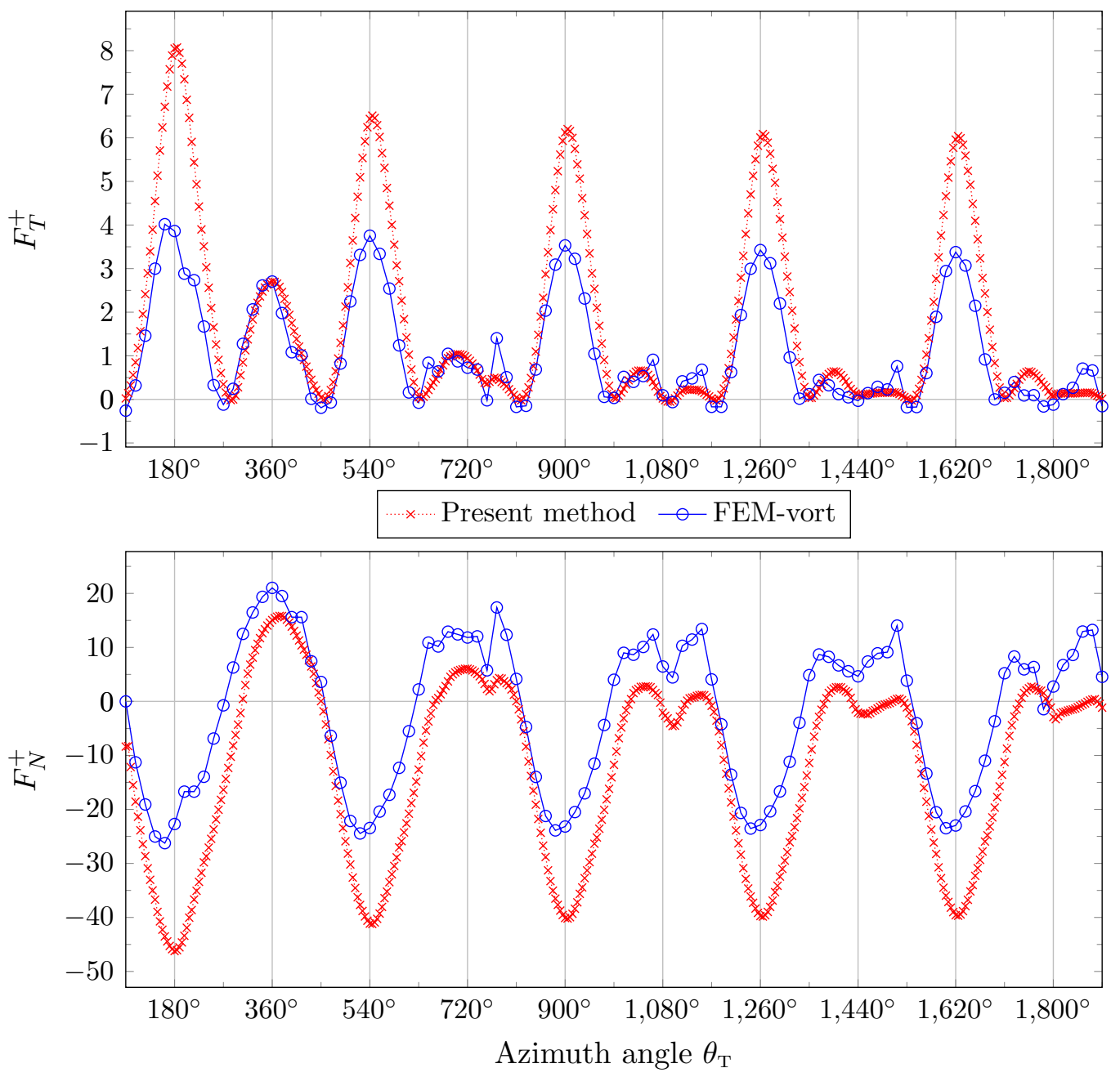

Figure 4.7: Comparison of equator forces predicted by the present method and the semi-empirical FEM-Vort method [6]

At downwind azimuth positions, the blade passes through a region of strong turbulence created by the wakes of the rotor blades and therefore reduces the average $F_{T}^{+}$values to nearly zero. In the absence of wake effects, the virtual camber and virtual incidence angle reduce the lift created by the blade at downwind positions, and would reduce the viscous drag for physical flows.

The time history of $F_{N}^{+}$predicted by the present method shows better agreement with FEM-Vort, perhaps owing to the minimal contribution of viscous drag in the normal direction. The present method's more negative prediction of $F_{N}^{+}$may be 
explained by the virtual camber and incidence angle. Both $F_{T}^{+}$and $F_{N}^{+}$show diminishing values over time with similar trends as those produced by FEM-Vort while in the downwind region of the VAWT.

\subsubsection{Aeroacoustic Validation}

Kelley et al. [37] carried out acoustic measurements on the Sandia 17-metre Troposkein VAWT, which is described in Table 4.1 and Fig. 4.1. These acoustic measurements used microphones around the VAWT to capture the total acoustic noise while the VAWT was in operation.

By predicting only the thickness and loading acoustic effects of the rotor blades, the present method is expected to primarily capture acoustic noise at frequencies ranging from one to several times the rotor's BPF, with the BPF of Sandia 17-metre turbine being $1.6 \mathrm{~Hz}$. As such, most of the acoustic noise will be below the $20 \mathrm{~Hz}$ threshold of human hearing and will have an insignificant SPL if expressed as decibels using A-weighting, as is done for several of the plots in [37].

Kelley et al. present their results for microphone (observer) positions 1.5 and 3 diameters downwind from the turbine axis (at unknown elevations) using acoustic pressure PSD plots. These plots describe the frequency content of the sound pressure level in terms of decibels per Hertz, and are useful for observing impulsive-type sound.

Figure 4.8 shows the PSD results from [37] for both observer positions and the results obtained using the present method for similar observer positions, at elevations of $1.8 \mathrm{~m}$ above the ground ( $13.475 \mathrm{~m}$ below the equator).

At the first multiple of $\mathrm{BPF}$, the present method predicts the PSD at to be approximately $15 \mathrm{~dB} / \mathrm{Hz}$ less than the experimental results for the observer $1.5 d_{\mathrm{T}}$ away, and $23 \mathrm{~dB} / \mathrm{Hz}$ less than the experimental results for the observer $3.0 d_{\mathrm{T}}$ away.

As the frequency increases, the discrepancy between SPL content increases. This 


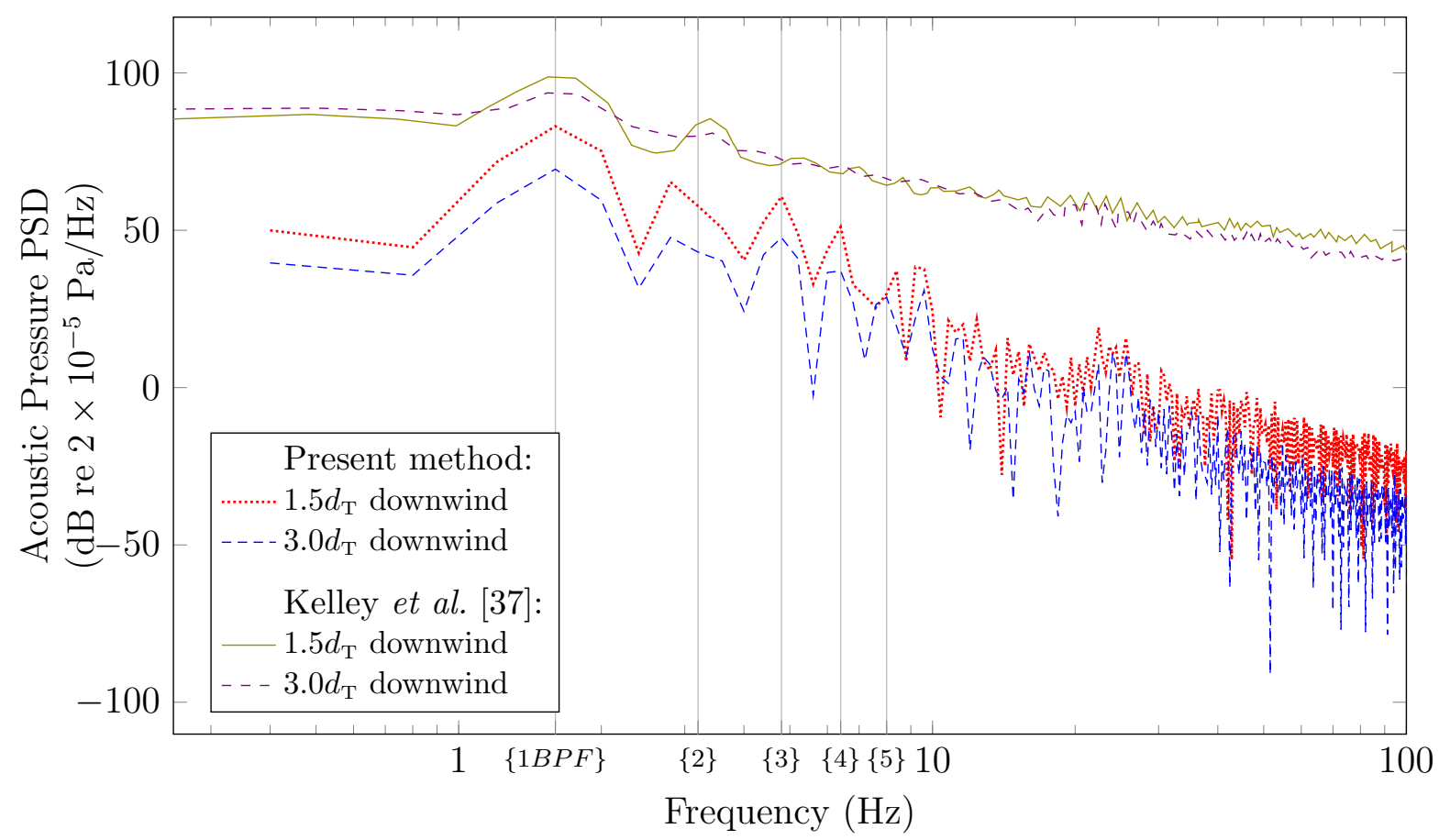

Figure 4.8: Comparison of PSD of the Sandia 17-m VAWT between experimental and present method results

trend is expected, as the present method is unable to capture high-frequency acoustic noise at high frequencies which are caused by mechanical sources, quadrupole effects, and the vorticity induced by the wake of the rotor blades. Both the experimental results and the present method's results appear to show an increased PSD between the frequencies of 18 and $30 \mathrm{~Hz}$, the cause of which is not considered here.

It is noted that the under-prediction of the SPL content, including at BPF harmonics, was expected. The fact that additional acoustic sources, such as quadrupole and mechanical sources, are not considered does impact the SPL predictions. However, it is expected that this method accurately predicts the VAWT aeroacoustic noise due to the thickness and loading effects. 


\subsection{Parametric Investigations}

The aerodynamic and aeroacoustic programs developed in the present work allow for numerous investigations to be performed into aerodynamics and aeroacoustic fields associated with numerous combinations of VAWT geometric and operational parameters.

This section presents aerodynamic and aeroacoustic results for different rotor blade types, geometric scales, number of rotor blades, and TSRs. Table 4.3 summarizes the differing aspects of the test cases, Table 4.4 contains the parameters which are constant for every case, and Fig. 4.9 illustrates the profile of the basic Troposkein rotor being considered here.

Unless otherwise specified, acoustic observer distances from the VAWT rotors are measured from the turbine's axis of rotation and are level with the equator of the rotor.

\begin{tabular}{|c|c|c|c|c|}
\hline Section & Rotor geometry & Turbine diameter & Number of blades & TSR \\
\hline \hline 4.3 .1 & Troposkein, Straight & $1 \mathrm{~m}$ & 2 & 5 \\
\hline 4.3 .2 & Troposkein & $1,5,10,25 \mathrm{~m}$ & 2 & 5 \\
\hline 4.3 .3 & Troposkein & $1 \mathrm{~m}$ & $2,3,4$ & 5 \\
\hline 4.3 .4 & Troposkein & $1 \mathrm{~m}$ & 2 & $3,4,5,6$ \\
\hline
\end{tabular}

Table 4.3: Overview of the parameters being investigated

\subsubsection{Effects due to Different Rotor Geometries}

Aerodynamic and aeroacoustic simulations of two similar rotors with different geometries are considered. In addition to the Troposkein rotor, a straight-blade rotor with equivalent diameter, swept area, and solidity ratio was considered.

Figure 4.10 contains plots of the non-dimensional tangential force $F_{T}^{+}$over the 


\begin{tabular}{|l|c|}
\hline Parameter & Value \\
\hline \hline Rotor solidity $\sigma_{\mathrm{T}}$ & 0.12 \\
\hline Wind speed $U_{\infty}$ & $9 \mathrm{~m} / \mathrm{s}$ \\
\hline Azimuth increment $\Delta \theta_{\mathrm{T}}$ & $4.5^{\circ}$ \\
\hline Span-wise discretization $N_{h}$ & 17 \\
\hline Airfoil discretization $N_{a}$ & 50 \\
\hline
\end{tabular}

Table 4.4: Constants throughout all investigations

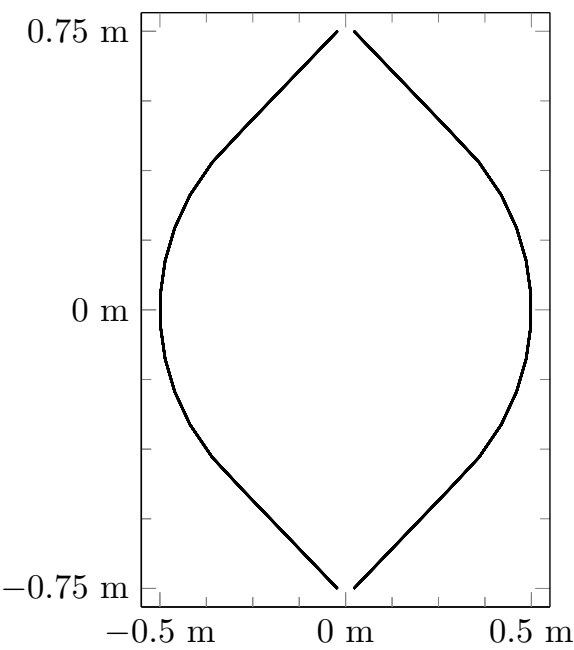

Figure 4.9: Illustration of the basic Troposkein turbine considered in the present investigations

height and azimuth of the Troposkein and straight-blade rotors. It can be seen that both turbines experience the largest values of $F_{T}^{+}$, with the peak values for the Troposkein rotor being greater than the straight-blade rotor. The ends of the Troposkein rotor blades do not contribute significant tangential force due to their low local speed ratio. Both rotors produce significantly less tangential force when downwind primarily due to the wake effects, where the wake is visualized in Fig. 4.11 and Fig. 4.12. The VPs are visualized at $1 / 4$ scale relative to the rotors with darker shades representing stronger vortex intensity.

The aeroacoustics of the Troposkein and straight-blade rotors are compared using 
the SPL directivity plots in Fig. 4.13 for observer positions $3 d_{\mathrm{T}}$ away around the equator of the rotors. The acoustic radiation patterns have different shapes, though both are greatest approximately upwind from the rotor, with the SPL around a Troposkein rotor being 6 to $8.5 \mathrm{~dB}$ less than the straight-blade rotor. This reduction may be due to destructive interference between the acoustics emissions of the blades, though more detailed investigations are needed to confirm this hypothesis.

Figure 4.14 considers PSD for an observer $3 d_{\mathrm{T}}$ upwind, and shows that the SPL of the straight-blade rotor is primarily due to frequencies less than $2 B P F$.

\subsubsection{Effects due to Varying Rotor Scale}

The basic Troposkein VAWT was scaled up to diameters of $5 \mathrm{~m}, 10 \mathrm{~m}$, and $25 \mathrm{~m}$, representing the range of common sizes. Scaling maintains the swept area and solidity ratio.

Figure 4.15 shows the SPL at various distances away from the VAWTs. To maintain similarity in the results, distances are expressed in terms of rotor diameters $\left(d_{\mathrm{T}}\right)$. All plots are in excellent agreement and are nearly indistinguishable, indicating that the SPL at a given number of rotor diameters away is independent of the rotor's size. Beyond approximately $10 d_{\mathrm{T}}$ away, the SPL decreases following a spherical divergence trend ( $-6 \mathrm{~dB}$ per doubling of distance). Therefore, rotor's near-field acoustic effects are likely limited to a distance of $10 d_{\mathrm{T}}$ from the rotor.

\subsubsection{Effects due to the Number of Rotor Blades}

The number of blades on the Troposkein rotor was increased for cases with 3 and 4 rotor blades, while reducing the chord length to maintain the rotor solidity ratio according to Eq. (1.1). 


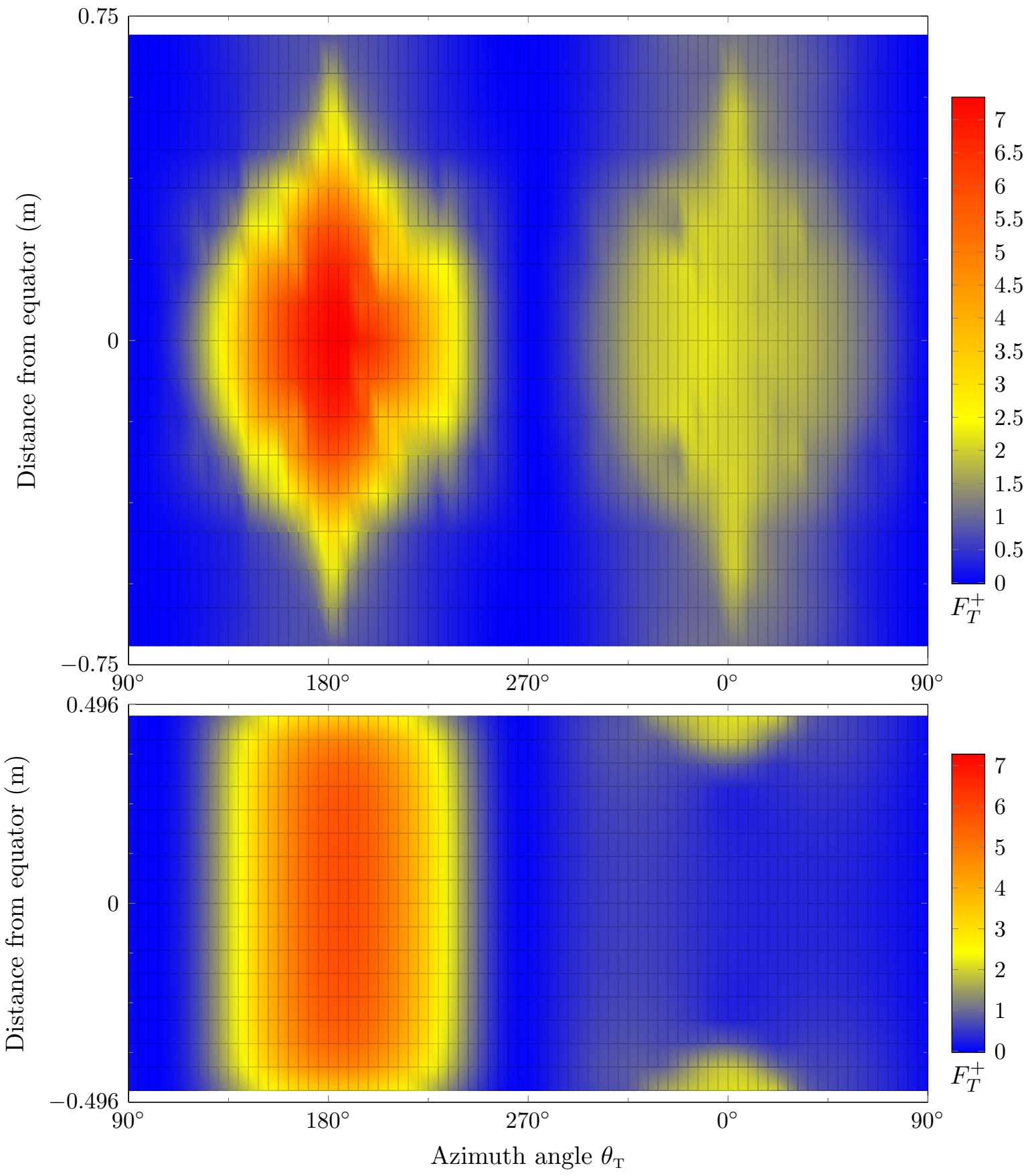

Figure 4.10: Non-dimensional tangential force $F_{T}^{+}$over the height a Troposkein blade (top) and a straight blade (bottom) throughout one rotation 

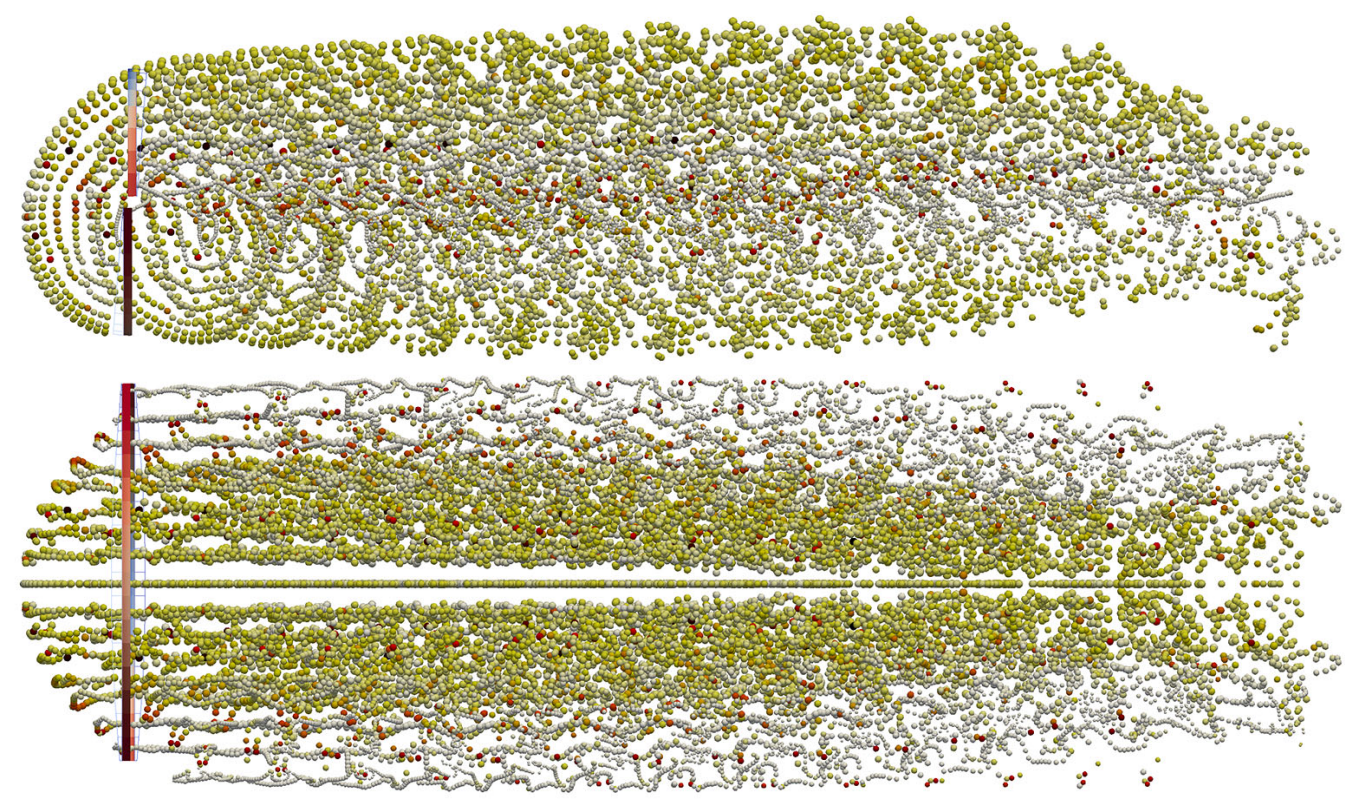

Figure 4.11: Top (top) and side (bottom) views of a visualization of the Troposkein rotor and its VP wake
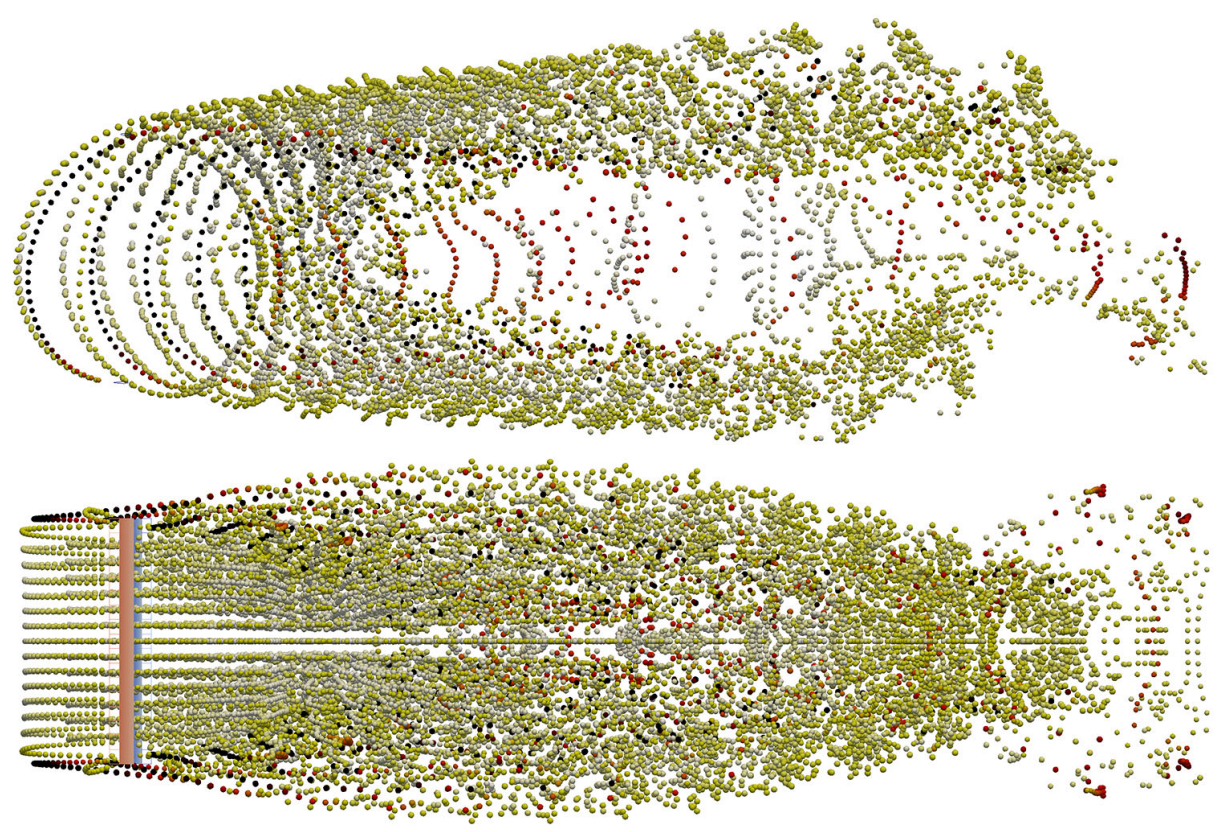

Figure 4.12: Top (top) and side (bottom) views of a visualization of a straight-blade rotor and its VP wake 


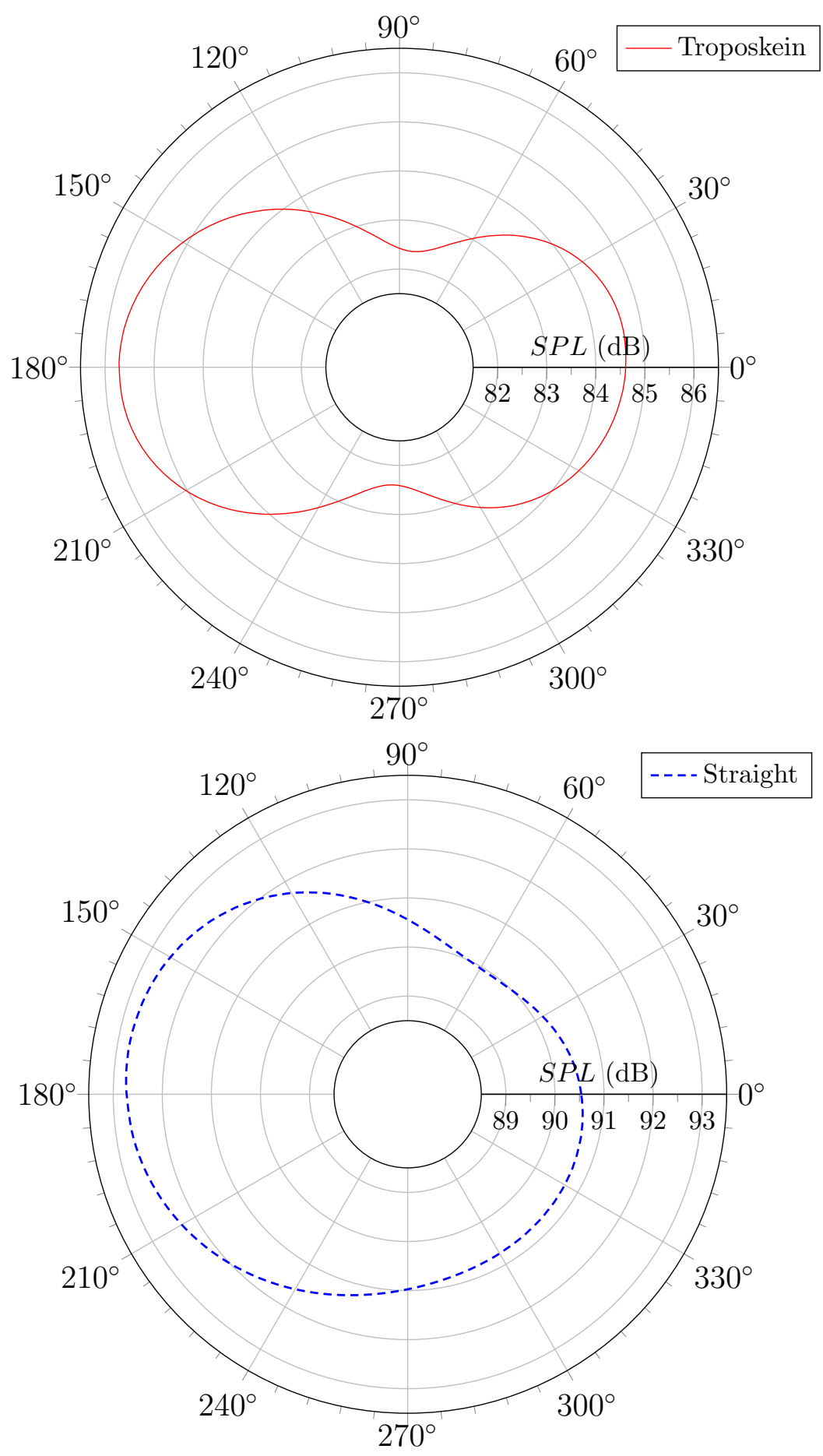

Figure 4.13: SPL directivity plots for Troposkein and straight-blade rotors at an observer distance of $3 d_{\mathrm{T}}$ 


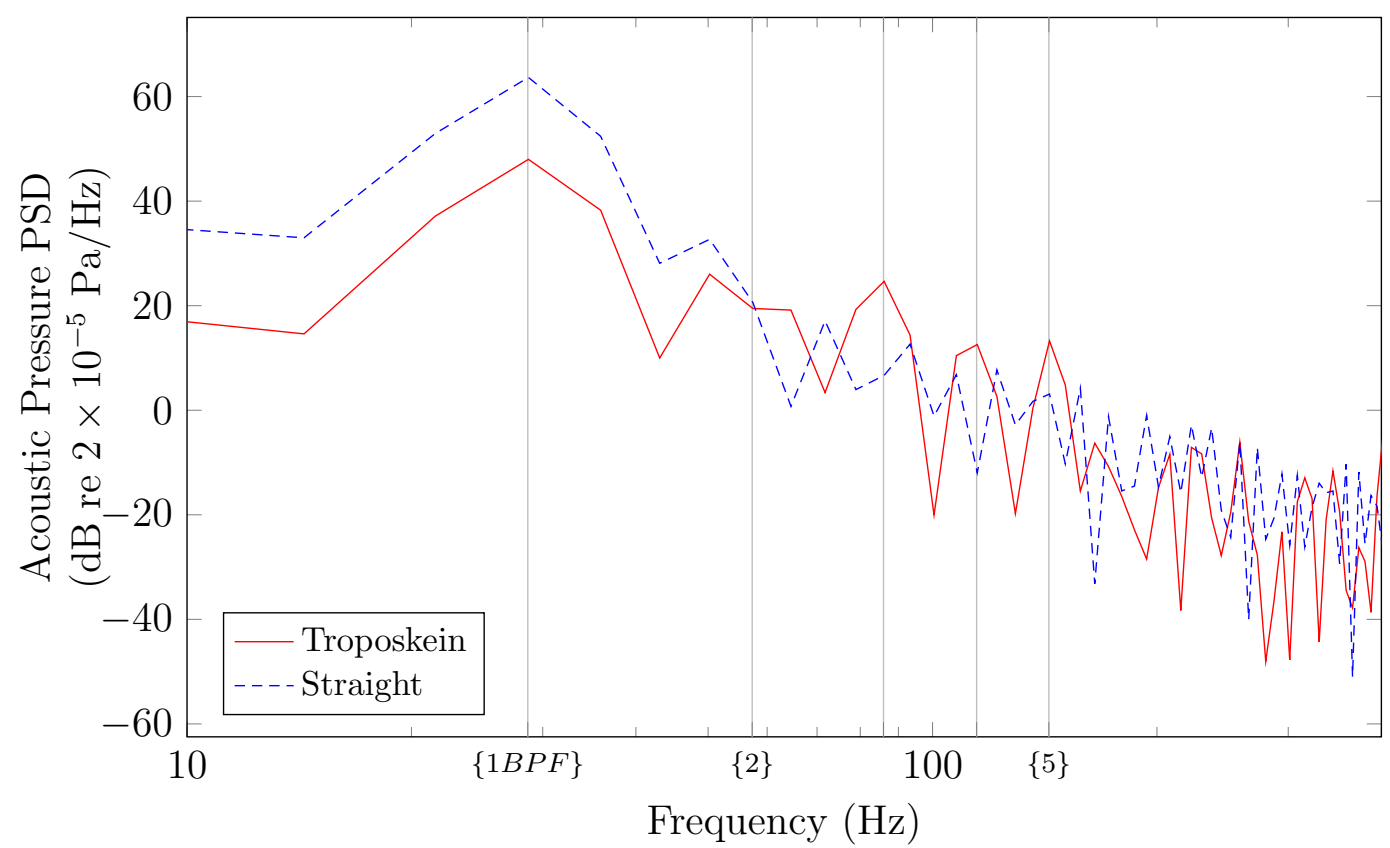

Figure 4.14: Comparison of the PSD of a Troposkein geometry and a straight-bladed geometry for an observer $3 d_{\mathrm{T}}$ upwind from the turbine axis

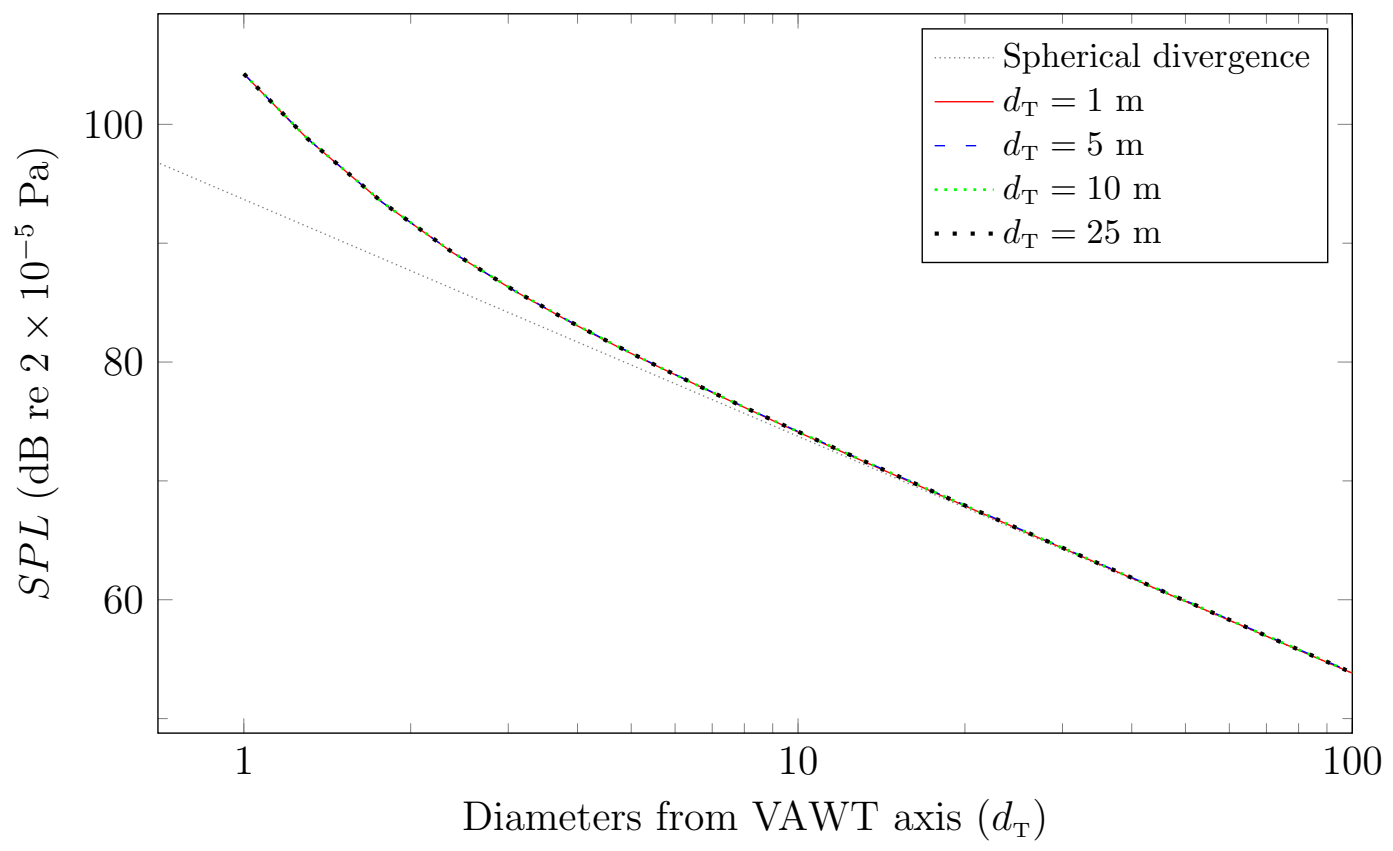

Figure 4.15: SPL of various geometric scales of Troposkein VAWTs for observers at varying distances upwind from the turbine axis 


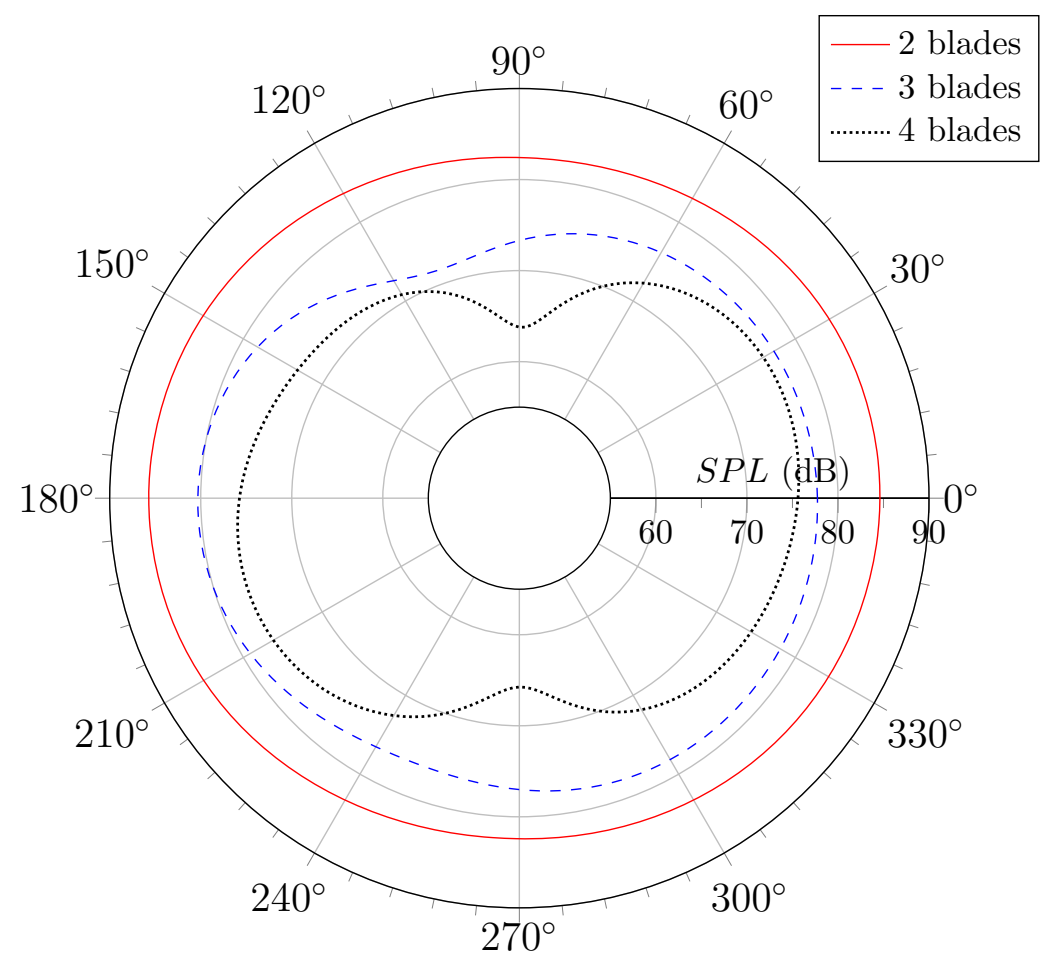

Figure 4.16: SPL directivity plots for Troposkein rotors with various numbers of blades

Figure 4.16 shows the directivity plots for the SPL radiation pattern of each rotor, with the SPL decreasing for an increasing number of blades and a radiation pattern shape for each rotor. The observer positions for these plots were 3 rotor diameters from the turbine axis and level with the rotor equator. This result is perhaps surprising as the additional blade(s) create additional wake to interact with other blades, which would create pressure fluctuations over the surface and in turn add to the loading acoustic pressure. It may be that the smoothing of the wake (due to the VP smoothing radii) is too great for additional wake to create any significant changes, and perhaps the observed reduction in SPL is more dependent on the shorter rotor blade chord length. 


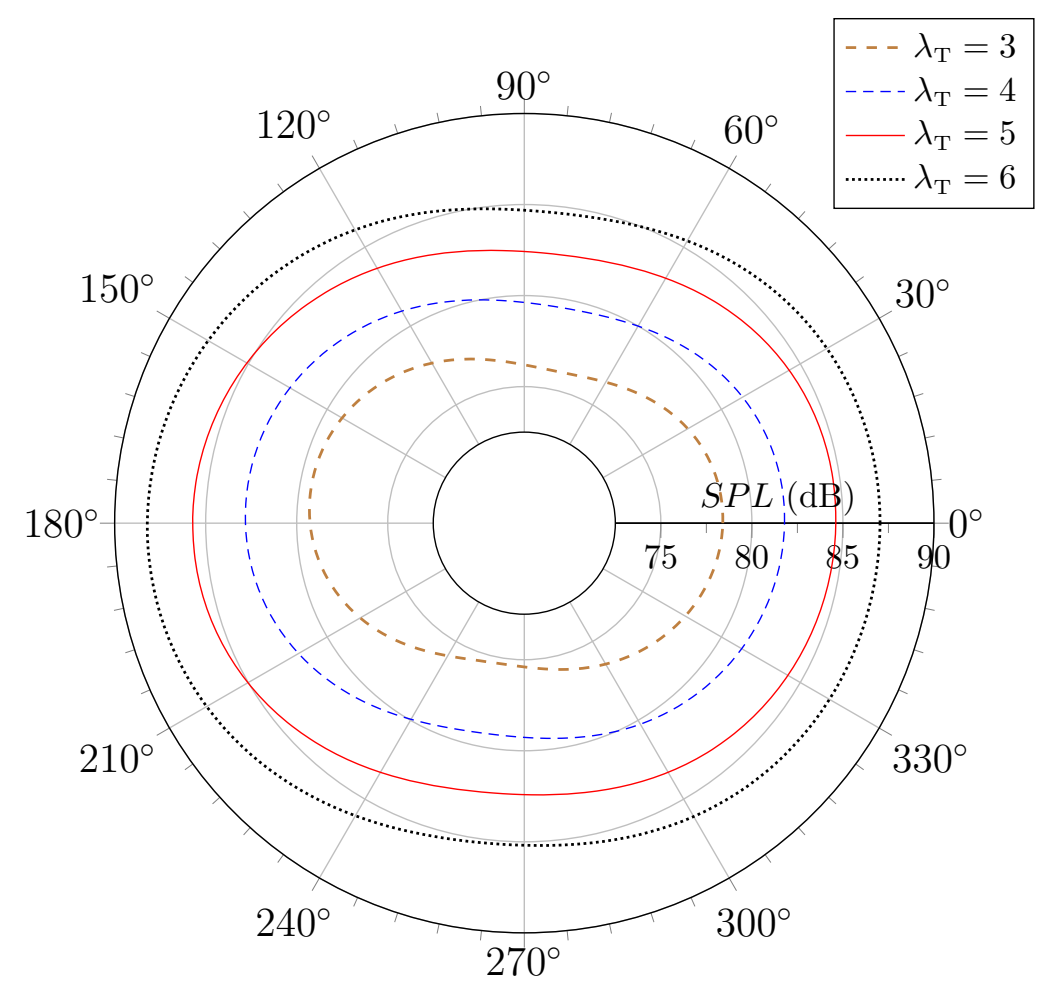

Figure 4.17: SPL directivity plots for a Troposkein rotors operating at various TSRs

\subsubsection{Effects due to Tip Speed Ratio}

The TSR is a relationship between the free-stream velocity and the rotor's radius and angular velocity, and is defined in Eq. (1.3). Since these cases consider a constant wind speed of $9 \mathrm{~m} / \mathrm{s}$ to maintain similar environmental conditions, and an unchanging rotor radius of $0.5 \mathrm{~m}$ (1.0 $\mathrm{m}$ diameter $)$, the angular velocity of the rotor is changed to vary the tip speed ratio. A range in TSR between the values of 3 and 6 is considered here.

The SPL directivity plots in Fig. 4.17 are for observers at a distance of 3 rotor diameters from the turbine's axis, and show a reduction in SPL of approximately 2 to $3 \mathrm{~dB}$ per integer decrease of TSR around the entire azimuth.

An increase in TSR corresponds to an increase in velocity along the rotor blades 
which is also fluctuating at an increased rate, and a larger fluctuation in the Mach number in the acoustic radiation direction $\left(M_{R}\right)$. Furthermore, the greater angular velocity of the turbine at high TSRs causes the pressure distribution over the blades to fluctuate more rapidly. Inspection of Eq. (3.10) and (3.11) and the contributing Eq. (3.2) and (3.3) reveals that these changes will cause the both thickness and loading perturbation pressures to fluctuate between larger values, thereby inducing a larger SPL around the rotor. 


\section{Chapter 5}

\section{Summary, Conclusions, and Recommendations}

\subsection{Summary and Contributions}

The aeroacoustics associated with vertical-axis wind turbines are not particularly well-characterized, with few publications on the subject. The present work developed a prediction tool which provides insight into the aerodynamics and aeroacoustics of VAWT rotor blades with minimal computational cost.

The aeroacoustics are fundamentally related to the aerodynamics of the problem, and vertical-axis wind turbine rotor blades experience very complex aerodynamics including curvilinear and highly turbulent flows. The first major contribution of this work is a subsonic aerodynamic solver which uses an unsteady inviscid panel method over the surface of rigid lifting bodies and vortex particles to represent the wake created by the bodies. This aerodynamic solver may be used to obtain the pressure distribution over VAWT rotor blades. 
The second major contribution is an aeroacoustic solver which uses a new implementation of Formulation 1C of the Ffowcs Williams-Hawkings equation for calculating the thickness and loading perturbation pressures over non-penetrable bodies. The acoustic predictions for blade thickness and loading components are successfully obtained. To the author's knowledge, the present combination of the aerodynamic and aeroacoustic methodologies is novel.

The third major contribution is the investigations into the effects of VAWT rotor parameters - including the blade geometry, the geometric scale, the number of blades, and the tip speed ratio - on the aeroacoustics around the rotor.

\subsection{Conclusions}

The inviscid aerodynamic methods implemented in the present work have been shown to agree well with expected results for fundamental cases including chord-wise pressure distribution, span-wise lift distribution, and unsteady lift coefficient for a pitching airfoil. The perturbation pressures due to acoustic monopole and dipole sources have been shown to be well-predicted by the non-penetrable form of Formulation 1C.

Viscous drag forces are not considered using the present method. The absence of drag forces causes an over-prediction in tangential force produced by the vertical-axis wind turbine rotor, though the vortex particle representation of the wake is effective at representing the turbulent flow effects over the downwind region of the VAWT.

The present method only captures aeroacoustic noise due to thickness and loading effects over the rotor blades. This aeroacoustic noise comprises a small portion of the total acoustic noise associated with an operating VAWT, and is typically dominated by frequencies at small multiples of the blade passage frequency.

Investigations into the thickness and loading noise of VAWT rotors found that 
Troposkein rotor geometries have acoustic sound pressure levels which are approximately $7 \mathrm{~dB}$ less than similar rotors with straight blades, though both have the greatest SPLs upwind of the rotor.

The acoustics at a given observer distance, non-dimensionalized by the rotor diameter, were found to be insensitive to the rotor scale, and near-field acoustic noise was found to be limited to approximately 10 rotor diameters from the turbine.

Increasing the number of rotor blades while maintaining the rotor solidity ratio reduces the SPL around the rotor, though the degree of reduction varies over the azimuth. Additionally, decreasing the tip speed ratio of the rotor by one integer value reduces the SPL by approximately 2 to $3 \mathrm{~dB}$ around the rotor.

\subsection{Recommendations}

The VAWT investigations presented in this work are a small sample of the possible results which may be obtained from the output of the aerodynamic and aeroacoustic simulations.

Aerodynamic studies may be performed to predict the performance of VAWT rotors and other rigid lifting bodies in motion, and aeroacoustic investigations may be performed using the results of those studies. The aeroacoustic implementation may be used as-is to perform investigations into the contributions of the separate regions of the rotor blades over a revolution in order to better understand the impacts of VAWT rotor design on thickness and loading acoustic noise generation.

Investigations which may be considered for future work include studying the effects of solidity ratio, rotor height-to-diameter ratio, and other rotor geometries such as rotor blades which spiral around the turbine axis and cambered airfoils.

There are many possible modifications and additions which may be incorporated 
into the present work to include the effects of factors which have thus far been neglected. Modelling physical bodies near the rotor, extending the aerodynamic model to address viscous flow phenomena, and considering the structural deformations of the rotor blades are all possible straight-forward extensions to the present work. Aeroacoustic analyses which include the quadrupole effects would capture a greater amount of the total acoustic noise, though a more detailed representation of the aerodynamics would be required.

The central tower of VAWTs may be aerodynamically modelled as a non-lifting body using only constant-source panels, and will likely create a separation in the wake produced while the rotor blades are upwind in addition to adding its own contribution to the wake.

Viscous aerodynamic effects should be more carefully considered to capture viscous phenomena such as dynamic stall and flow separation. The ONERA model of dynamic stall is a semi-empirical model used to predict the unsteady loads on an airfoil that is experiencing dynamic stall [58], and has been successfully implemented into computational models such as GENUVP which use a similar aerodynamic model as the present work [59].

Rather than simplify the rotor geometry as rigid, the structural properties of the rotor blade may be considered and an aeroelastic component may be included in the present method to capture the structural vibrations in the rotor blades. The code FEM-Vort, developed by Fereidooni [6], presents such a method which uses linear structural dynamic equations developed by Nitzsche [60] for Troposkein rotors and may be incorporated into the present aerodynamic analysis. The structural vibrations will contribute an additional fluctuation in the velocity distribution over the rotor blades which will contribute to the thickness perturbation pressure. 
Many environmental effects have largely been neglected in the present work. Atmospheric properties, including acoustic attenuation and wind shear, may be incorporated into the aerodynamic and aeroacoustic models to better understand their effects on the generation and propagation of sound.

Beyond additions to the present method to better capture the physical phenomena at work, optimizations to the aerodynamic code may significantly reduce the time required to perform simulations. The GENUVP code makes use of two numerical schemes which reduce the computational cost of the simulations from an order of the square of the number of surface panels $\left(N^{2}\right)$ and the square of the number of vortex particles $\left(N_{\mathrm{VP}}^{2}\right)$.

A subgrid approximation, as described in detail in [30], considers the rotor blades to have different scaling levels of discretization, and treats the aerodynamic effects of panels at a point far away from the panels as a group rather than individual panels. This subgrid approximation reduces the computational cost from $N^{2}$ to $N \log N$.

GENUVP similarly reduces the cost of the wake convection and deformation from $N_{\mathrm{VP}}{ }^{2}$ to $N_{\mathrm{vP}} \log N_{\mathrm{VP}}$ through the use of Particle-Mesh techniques which substitute direct particle-to-particle calculations with indirect calculations using a mesh of nodes throughout the domain [30].

Furthermore, an aerodynamic code which employs general-purpose computing on graphics processing units may provide a significant reduction in the computational time for highly discretized geometries since the majority of the calculations are independent and well-suited to graphics processing units which perform massively parallel computations compared to conventional multi-core central processing units. 


\section{List of References}

[1] H. J. Sutherland, D. E. Berg, and T. D. Ashwill, "A Retrospective of VAWT Technology," Tech. Rep. January, Sandia National Laboratories, Albuquerque, 2012.

[2] I. Paraschivoiu, Wind Turbine Design With Emphasis on Darrieus Concept. Montreal: Presses inter Polytechnique, 2002.

[3] F. Scheurich, Modelling the Aerodynamics of Vertical-Axis Wind Turbines. Phd, University of Glasgow, 2011.

[4] H. H. Hubbard and K. P. Shepherd, "Wind Turbine Acoustics." 1990.

[5] C. E. Pearson and W. Graham, "Investigation Of The Noise Sources On A Vertical Axis Wind Turbine Using An Acoustic Array," 19th AIAA/CEAS Aeroacoustics Conference, pp. 1-10, May 2013.

[6] A. Fereidooni, Numerical Study of Aeroelastic Behaviour of a Troposkien Shape Vertical Axis Wind Turbine. Master of applied science, Carleton University, 2013.

[7] M. M. Aslam Bhutta, N. Hayat, A. U. Farooq, Z. Ali, S. R. Jamil, and Z. Hussain, "Vertical axis wind turbine: A review of various configurations and design techniques," Renewable and Sustainable Energy Reviews, vol. 16, pp. 1926-1939, May 2012.

[8] G. Paumier, "Eoliennes gaspesie." http://commons.wikimedia.org/wiki/ File:Eoliennes_Gaspesie.jpg, 2005. Accessed: 2014-11-05.

[9] J. Montgomery, "VAWT on the vineyard: Small wind revisited." http://www.renewableenergyworld.com/rea/blog/post/2013/11/ vawt-on-the-vineyard-small-wind-revisited, 2013. Accessed: 201411-05. 
[10] Hydro-Québec, "History of Wind Power in Québec." http://www .hydroquebec . com/learning/eolienne/historique-eolien-hydro-quebec.html, 2014. Accessed: 2014-09-25.

[11] A. Adamek and S. Tudor, "A Brief History of Wind Power Development in Canada 1960s-1990s," 2010.

[12] X. Sun, D. Huang, and G. Wu, "The current state of offshore wind energy technology development," Energy, vol. 41, pp. 298-312, May 2012.

[13] S. Oerlemans, P. Sijtsma, and B. Méndez López, "Location and quantification of noise sources on a wind turbine," Journal of Sound and Vibration, vol. 299, pp. 869-883, Feb. 2007.

[14] K. Persson Waye and E. Öhrström, "Psycho-Acoustic Characters of Relevance for Annoyance of Wind Turbine Noise," Journal of Sound and Vibration, vol. 250, pp. 65-73, Feb. 2002.

[15] P. a. de Ruído, Wind Turbine Noise Prediction. Master, Universidade Tecnica de Lisboa, 2008.

[16] J. P. Harrison, "Wind Turbine Noise," Bulletin of Science, Technology \& Society, vol. 31, pp. 256-261, July 2011.

[17] A. J. Bedard and T. M. Georges, "Atmospheric Infrasound," Physics Today, vol. 53, no. 3, pp. 32-37, 2000.

[18] P. G. Migliore, W. P. Wolfe, and J. B. Fanucci, "Flow Curvature Effects on Darrieus Turbine Blade Aerodynamics," Journal of Energy, vol. 4, no. 2, pp. 4955,1980 .

[19] B. F. Blackwell, W. N. Sullivan, R. C. Reuter, and J. F. Banas, "Engineering Development Status of the Darrieus Wind Turbine," Journal of Energy, vol. 1, no. 1, pp. 50-64, 1977.

[20] J. Delfs, "Grundlagen der Aeroakustik (Basics of Aeroacoustics)." 2012.

[21] M. S. Howe, Acoustics of Fluid-Structure Interations. Cambridge: Cambridge University Press, 1998.

[22] M. J. Lighthill, "On sound generated aerodynamically. I. General theory," in Proceedings of the Royal Society London, Series A, (London), pp. 564-587, 1952. 
[23] M. S. Howe, Theory of Vortex Sound. Cambridge: Cambridge University Press, 2003.

[24] K. S. Brentner and F. Farassat, "An analytical comparison of the acoustic analogy and Kirchhoff Formulation for moving surfaces," AIAA Journal, vol. 36, no. 8, pp. 1379-1386, 1998.

[25] K. S. Brentner, "Modeling Aerodynamically Generated Sound : Recent Advances in Rotor Noise Prediction," in 38th Aerospace Sciences Meeting \& Exhibit, AIAA, no. January, (Reno, Nevada), 2000.

[26] J. E. Ffowcs Williams and D. L. Hawkings, "Sound Generation by Turbulence and Surfaces In Arbitrary Motion," Philosophical Transactions of the Royal Society of London. Series A, Mathematical and Physical Sciences, vol. 264, no. 1151, pp. 321-342, 1969.

[27] F. Farassat, "Derivation of Formulations 1 and 1A of Farassat," Tech. Rep. March, NASA Langley Research Center, Hampton, Virginia, 2007.

[28] F. Farassat and G. P. Succi, "A review of propeller discrete frequency noise prediction technology with emphasis on two current mathods for time domain calculations," Journal of Sound and Vibration, vol. 71, no. 3, pp. 399-419, 1980.

[29] V. L. Wells and A. Y. Han, "Acoustics of a moving source in a moving medium with application to propeller noise," Journal of Sound and Vibration, vol. 184, no. 4, pp. 651-663, 1995.

[30] D. G. Opoku, Aeroelastic and Aeroacoustic Modelling of Rotorcraft. PhD thesis, Carleton University, 2002.

[31] A. Tadamasa and M. Zangeneh, "Numerical prediction of wind turbine noise," Renewable Energy, vol. 36, pp. 1902-1912, July 2011.

[32] F. Farassat, "Theory of noise generation from moving bodies with an application to helicopter rotors," Tech. Rep. December, NASA Langley Research Center, Hampton, Virginia, 1975.

[33] A. Najafi-Yazdi, G. A. Bres, and L. Mongeau, "An acoustic analogy formulation for moving sources in uniformly moving media," Proceedings of the Royal Society A: Mathematical, Physical and Engineering Sciences, vol. 467, pp. 144-165, June 2010. 
[34] K. S. Brentner and F. Farassat, "Modeling aerodynamically generated sound of helicopter rotors," Progress in Aerospace Sciences, vol. 39, pp. 83-120, Feb. 2003.

[35] T. F. Brooks, D. S. Pope, and M. A. Marcolini, Airfoil self-noise and prediction, vol. 1218. National Aeronautics and Space Administration, Office of Management, Scientific and Technical Information Division, 1989.

[36] J. Casper and F. Farassat, "A new time domain formulation for broadband noise predictions," International Journal of Aeroacoustics, vol. 1, no. 3, pp. 207-240, 2002.

[37] N. D. Kelley, R. R. Hemphill, and D. L. Sengupta, "Television interference and acoustic emissions associated with the operation of the Darrieus VAWT," in Proceedings of the 5th Biennial Wind Energy Conference and Workshop, vol. 1, pp. 397-413, 1981.

[38] C. Pearson, Vertical Axis Wind Turbine Acoustics. Phd, Cambridge University, 2013.

[39] J. H. Strickland, B. T. Webster, and T. Nguyen, "A Vortex Model of the Darrieus Turbine: An Analytical and Experimental Study," tech. rep., Sandia Laboratories, 1980.

[40] M. Hansen, J. Sørensen, S. Voutsinas, N. Sørensen, and H. Madsen, "State of the art in wind turbine aerodynamics and aeroelasticity," Progress in Aerospace Sciences, vol. 42, pp. 285-330, June 2006.

[41] G.-H. Cottet and P. Koumoutsakos, Vortex Methods: Theory and Practice. Cambridge: The Press Syndicate of the University of Cambridge, 2000.

[42] K. R. Dixon, The Near Wake Structure of a Vertical Axis Wind Turbine. M. sc., Delft University of Technology, 2008.

[43] J. L. Hess and A. M. O. Smith, "Calculation of non-lifting potential flow about arbitrary three-dimensional bodies." 1962.

[44] J. Hess and A. Smith, "Calculation of potential flow about arbitrary bodies," Progress in Aerospace Sciences, vol. 8, pp. 1-138, Jan. 1967.

[45] J. L. Hess, "Calculation of potential flow about arbitrary three-dimensional lifting bodies." 1972. 
[46] J. Katz and A. Plotkin, Low-Speed Aerodynamics. New York: Cambridge University Press, 2nd ed., 2001.

[47] J. D. Anderson, Fundamentals of Aerodynamics. McGraw-Hill Education, 5th ed., 2010.

[48] S. G. Voutsinas, "Vortex methods in aeronautics: how to make things work," International Journal of Computational Fluid Dynamics, vol. 20, no. 1, pp. 3-18, 2006.

[49] J. T. Beale and A. Majda, "High order accurate vortex methods with explicit velocity kernels," Journal of Computational Physics, vol. 58, pp. 188-208, Apr. 1985.

[50] R. Williams, J. Rocha, E. Matida, and F. Nitzsche, "IMECE2014-38199," in Proc. of the ASME 2014 International Mechanical Engineering Congress \& Exposition, 2014.

[51] D. J. Lee and S. U. Na, "Numerical simulations of wake structure generated by rotating blades using a time marching, free vortex blob method," European Journal of Mechanics - B/Fluids, vol. 18, pp. 147-159, Jan. 1999.

[52] M. Drela, "XFOIL subsonic airfoil development system." http://web.mit.edu/ drela/Public/web/xfoil/, 2013. Accessed: 2014-11-05.

[53] W. J. McCroskey, K. W. McAlister, L. W. Carr, S. L. Pucci, O. Lambert, and R. F. Indergrand, "Dynamic Stall on Advanced Airfoil Sections," Journal of the American Helicopter Society, vol. 26, no. 3, pp. 40-50, 1981.

[54] H. Kuttruff, Acoustics: An Introduction. Abingdon: Taylor and Francis, 1 ed., 2007.

[55] D. Casalino, "An advanced time approach for acoustic analogy predictions," Journal of Sound and Vibration, vol. 261, pp. 583-612, Apr. 2003.

[56] R. O. Nellums, "Design Characterisitcs of the DOE/ALO-Alcoa 17 Meter Turbine," in Proceedings of the Vertical Axis Wind Turbine (VAWT) Design Technology Seminar for Industry (S. F. Johnston, ed.), (Albuquerque, New Mexico), pp. 67-76, 1982. 
[57] E. G. Kadlec, "Current and Future Design Characterisitcs of Vertical Axis Wind Turbines," in Proceedings of the Vertical Axis Wind Turbine (VAWT) Design Technology Seminar for Industry (S. F. Johnston, ed.), (Albuquerque, New Mexico), pp. 45-54, Sandia National Laboratories, 1982.

[58] K. W. McAlister, O. Lambert, and D. Petot, "Application of the ONERA Model of Dynamic Stall," tech. rep., NASA Ames Research Center, Moffett Field, CA, 1984.

[59] G. L. Davis, Trailing Edge Flap Control of Dynamic Stall on Helicopter Rotor Blades. Master of applied science, Carleton University, 2005.

[60] F. Nitzsche, Aeroelastic Analysis Of A Darrieus Type Wind Turbine Blade With Troposkien Geometry. Doctor of philosophy, Standford University, 1983. 


\section{Appendix A}

\section{Equations for Aerodynamic Singularity}

\section{Elements}

The singularity elements, or panels, used to represent the surface of the rotor blades and the near wake are quadrilaterals. That is, four-sided first-order shapes, meaning all lie in the same plane. The present work only considers singularity elements which have constant source and/or doublet strength (or "density") distributions.

When solving for the aerodynamics, both the scalar velocity potential and the velocity induced by the singularity elements must be determined. Hess and Smith [43-45] pioneered this approach to aerodynamic modelling, and presented solutions for such uniform-strength singularity elements. Katz and Plotkin [46] later summarized much of Hess and Smith's work, though a number of errors have been discovered in [46] which are pointed out here.

This appendix contains the equations used to solve for the velocity potential and induced velocity fields surrounding three-dimensional first-order panels with constant source and/or doublet strengths, with corrections to the errors found in [46]. While [43-46] consider only quadrilateral singularity elements, the equations presented here have been modified to be suitable for a first-order singularity element with two or 
more defining points.

\section{A.1 Three-dimensional quadrilateral panels}

Solving for a general first-order panel in three-dimensional space can be very complicated. To simplify the process, the points bounding the panel and the points of interest (where the velocity potential and/or induced velocity is to be calculated) are transformed from the global coordinate system $(X, Y, Z)$ to a local coordinate system $(x, y, z)$ in which all of the bounding points lie on the $x-y$ plane. As long as these conditions are met, the origin and orientation of the local coordinate system do not affect the results. For definiteness, the origin is taken as the centroid of the panel and the $x$-axis is oriented parallel to the vector from the first to second bounding points of the panel. The positive $z$-axis is oriented such that the bound points are numbered following the right-hand rule about the $z$-axis.

After solving for the induced velocities, the velocity vectors must be rotated back to the global coordinate system. The velocity potential is a scalar quantity, and does not require this re-transformation.

For a point $\left(x_{p}, y_{p}, z_{p}\right)$ being influenced by a panel with $q$ bounding points $\left(x_{1}, y_{1}, 0\right),\left(x_{2}, y_{2}, 0\right), \ldots,\left(x_{q}, y_{q}, 0\right)$ in the panel's local coordinate system, the following quantities are introduced:

$$
\begin{aligned}
d_{k, k+1} & =\sqrt{\left(x_{k+1}-x_{k}\right)^{2}+\left(y_{k+1}-y_{k}\right)^{2}}, \\
m_{k, k+1} & =\frac{y_{k+1}-y_{k}}{x_{k+1}-x_{k}}, \\
r_{k} & =\sqrt{\left(x_{p}-x_{k}\right)^{2}+\left(y_{p}-y_{k}\right)^{2}+z_{p}^{2}}, \\
e_{k} & =\left(x_{p}-x_{k}\right)^{2}+z_{p}^{2},
\end{aligned}
$$




$$
h_{k}=\left(x_{p}-x_{k}\right)\left(y_{p}-y_{k}\right),
$$

for $k=1 . . q$ and if $k=q+1$ then $k=1$. These quantities are used for both uniform-strength source and doublet panels.

\section{A.1.1 3D uniform-strength source panels}

For a 3D uniform-strength source panel with $q$ bounding points, the scalar velocity potential is

$$
\begin{gathered}
\Phi_{\sigma}=\frac{-\sigma}{4 \pi}\left\{\sum_{k=1}^{q}\left[\frac{\left(x_{p}-x_{k}\right)\left(y_{k+1}-y_{k}\right)-\left(y_{p}-y_{k}\right)\left(x_{k+1}-x_{k}\right)}{d_{k, k+1}} \ln \frac{r_{k}+r_{k+1}+d_{k, k+1}}{r_{k}+r_{k+1}-d_{k, k+1}}\right]\right. \\
\left.+z_{p} \sum_{k=1}^{q}\left[\arctan \left(\frac{m_{k, k+1} e_{k}-h_{k}}{z r_{k}}\right)-\arctan \left(\frac{m_{k, k+1} e_{k+1}-h_{k+1}}{z r_{k+1}}\right)\right]\right\} .
\end{gathered}
$$

Note this is different than [46] in that the $z_{p}$ factor in Eq. (A.6) before the second summation is not an absolute value.

The velocity induced by a source panel in the panel's local coordinate system is given by

$$
\left(\mathbf{u}_{\sigma}^{\prime}\right)_{x y z}=u_{\sigma x}^{\prime} \hat{\mathbf{e}}_{x}+u_{\sigma y}^{\prime} \hat{\mathbf{e}}_{y}+u_{\sigma z}^{\prime} \hat{\mathbf{e}}_{z},
$$


where

$$
\begin{aligned}
& u_{\sigma x}^{\prime}=\frac{\sigma}{4 \pi} \sum_{k=1}^{q}\left[\frac{y_{k+1}-y_{k}}{d_{k, k+1}} \ln \frac{r_{k}+r_{k+1}-d_{k, k+1}}{r_{k}+r_{k+1}+d_{k, k+1}}\right], \\
& u_{\sigma y}^{\prime}=\frac{\sigma}{4 \pi} \sum_{k=1}^{q}\left[\frac{x_{k+1}-x_{k}}{d_{k, k+1}} \ln \frac{r_{k}+r_{k+1}-d_{k, k+1}}{r_{k}+r_{k+1}+d_{k, k+1}}\right], \\
& u_{\sigma z}^{\prime}=\frac{\sigma}{4 \pi} \sum_{k=1}^{q}\left[\arctan \left(\frac{m_{k, k+1} e_{k}-h_{k}}{z_{p} r_{k}}\right)-\arctan \left(\frac{m_{k, k+1} e_{k+1}-h_{k+1}}{z_{p} r_{k+1}}\right)\right] .
\end{aligned}
$$

\section{A.1.2 3D uniform-strength doublet panels}

For a 3D uniform-strength doublet panel with $q$ bounding points and the constant doublet strength vector in the positive $z$-direction, the scalar velocity potential is

$$
\Phi_{\mu}=\frac{-\mu}{4 \pi} \sum_{k=1}^{q}\left[\arctan \left(\frac{m_{k, k+1} e_{k}-h_{k}}{z r_{k}}\right)-\arctan \left(\frac{m_{k, k+1} e_{k+1}-h_{k+1}}{z r_{k+1}}\right)\right] .
$$

Note this is the negative of the corresponding equation provided in [46].

A doublet panel with constant strength $\mu$ is equivalent to a vortex ring of strength $\mu$ around the perimeter. The velocity induced by a doublet panel may therefore be calculated as the sum of the velocities induced by the vortex filaments along each edge of the panel, expressed as

$$
\mathbf{u}_{\mu}^{\prime}=\sum_{k=1}^{q}\left[\frac{\mu}{4 \pi} \frac{\mathbf{r}_{p, k} \times \mathbf{r}_{p, k+1}}{\left|\mathbf{r}_{p, k} \times \mathbf{r}_{p, k+1}\right|^{2}} \mathbf{r}_{k+1, k} \cdot\left(\frac{\mathbf{r}_{p, k}}{r_{p, k}}-\frac{\mathbf{r}_{p, k+1}}{r_{p, k+1}}\right)\right]
$$

where $\mathbf{r}_{p, k}$ is the position vector of point $p$ relative to point panel bounding point $k$ (not to be confused with $r_{k}$ from Eq. (A.3). This velocity formulation can be performed in the global coordinate system, and therefore does not require any transformations 
to or from the panel's local coordinate system.

It is noted here that the velocity formulation in Eq. (A.9) is applicable to any closed vortex ring composed of linear vortex filaments, and can therefore be used to determine the perturbation velocity created by twisted uniform-strength doublet panels.

\section{A.2 Three-dimensional vortex particles}

The equation for the velocity induced at position $\mathbf{q}$ by a VP at position $\mathbf{Z}$ is found from $[48]$ as

$$
\mathbf{u}_{\mathrm{VP}}^{\prime}(\mathbf{q})=\frac{\boldsymbol{\xi} \times \mathbf{r}}{4 \pi r^{3}} \zeta_{\epsilon}(r),
$$

where $\boldsymbol{\xi}$ is the vector intensity of the vortex particle, $\zeta_{\epsilon}$ is the smoothing function, $\epsilon$ is the smoothing radius, and

$$
\begin{gathered}
\mathbf{r}=\mathbf{q}-\mathbf{Z}, \\
\zeta_{\epsilon}(r)=1-e^{-(r / \epsilon)^{3}} .
\end{gathered}
$$

The vortex particles are convected and deformed at every aerodynamic simulation time step. The rates of convection and deformation are determined from the material character at the position of each vortex particle [48]:

$$
\begin{gathered}
\frac{\mathrm{d} \mathbf{Z}_{p}(\tau)}{\mathrm{d} \tau}=\mathbf{u}\left(\mathbf{Z}_{p} ; \tau\right), \\
\frac{\mathrm{d} \boldsymbol{\xi}_{p}(\tau)}{\mathrm{d} \tau}=\left(\boldsymbol{\xi}_{p}(\tau) \nabla\right) \mathbf{u}\left(\mathbf{Z}_{p}, \tau\right)=\stackrel{\leftrightarrow}{D}\left(\mathbf{x}_{0}, \tau\right) \cdot \boldsymbol{\xi}_{p}(\tau),
\end{gathered}
$$

where

$$
\stackrel{\leftrightarrow}{D}=\left[D_{i j}\right]=\left[\frac{\partial u_{i}}{\partial x_{j}}\right]
$$


and the total fluid velocity $\mathbf{u}$ is the sum of the mean flow and the perturbation velocities:

$$
\begin{aligned}
\mathbf{u} & =\mathbf{U}_{\infty}+\mathbf{u}_{\text {solid }}^{\prime}+\mathbf{u}_{\text {near-wake }}^{\prime}+\mathbf{u}_{\text {far-wake }} \\
& =\mathbf{U}_{\infty}+\left(\mathbf{u}_{\sigma}^{\prime}+\mathbf{u}_{\mu}^{\prime}\right)+\left(\mathbf{u}_{\mu_{w}}^{\prime}\right)+\left(\mathbf{u}_{\mathrm{VF}}^{\prime}+\mathbf{u}_{\mathrm{VP}}^{\prime}\right) .
\end{aligned}
$$

The flow fields induced by vortex particles on neighbouring vortex particles are the primary contributing factor to the deformation rate. From [48], the rate of deformation of vortex particle intensity due to surrounding vortex particles is calculated using

$$
\left.(\boldsymbol{\xi} \nabla) \mathbf{u}_{\mathrm{VP}}^{\prime}\right|_{p}=\sum_{q}^{N_{\mathrm{VP}}}\left\{\frac{\boldsymbol{\xi}_{q} \times \boldsymbol{\xi}_{p}}{4 \pi r_{p q}^{3}}-3 \frac{\left(\boldsymbol{\xi}_{p} \mathbf{r}_{\mathbf{p q}}\right)\left(\boldsymbol{\xi}_{q} \times \mathbf{r}_{\mathbf{p q}}\right)}{4 \pi r_{q p}^{5}}\right\}
$$

with $\mathbf{r}_{\mathbf{p q}}=\mathbf{Z}_{p}-\mathbf{Z}_{q}$.

As described in [48], the contribution of $\mathbf{u}_{\text {solid }}^{\prime}$ to Eq. (A.14) is complicated and may be neglected. Similarly, the near-wake and far-wake vortex filaments do not have a significant contribution to the deformation of the far-wake vortex particles and are neglected as well.

\section{A.3 Transformation from doublet panels to vortex filaments and vortex particles}

In the transition between aerodynamic simulation time steps, the near-wake doublet panels and the far-wake vortex filaments (which are coincident with the trailing edges of the near-wake doublet panels) are convected away from the trailing edge, as shown in Fig. A.1.

The constant-strength near-wake doublet panels may be equivalently represented 


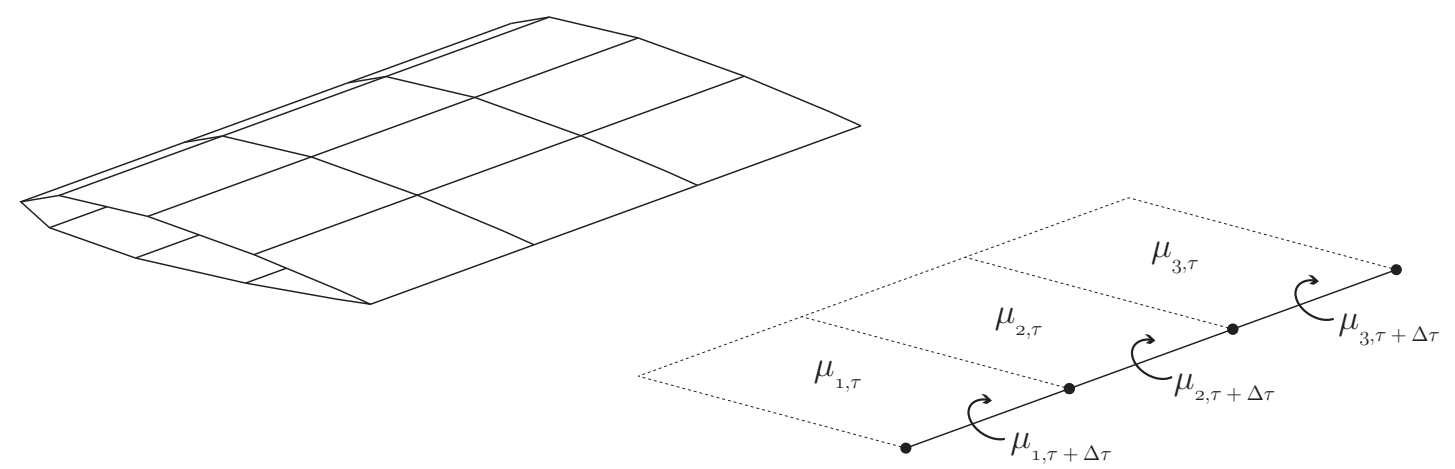

Figure A.1: Near-wake panels and vortex filaments from previous time step after being convected

by a ring of vortex filaments along the border with strengths equal to the doublet panel strength. The vortex filament strengths are combined with any other coincident vortex filaments through summation, as shown in Fig. A.2.

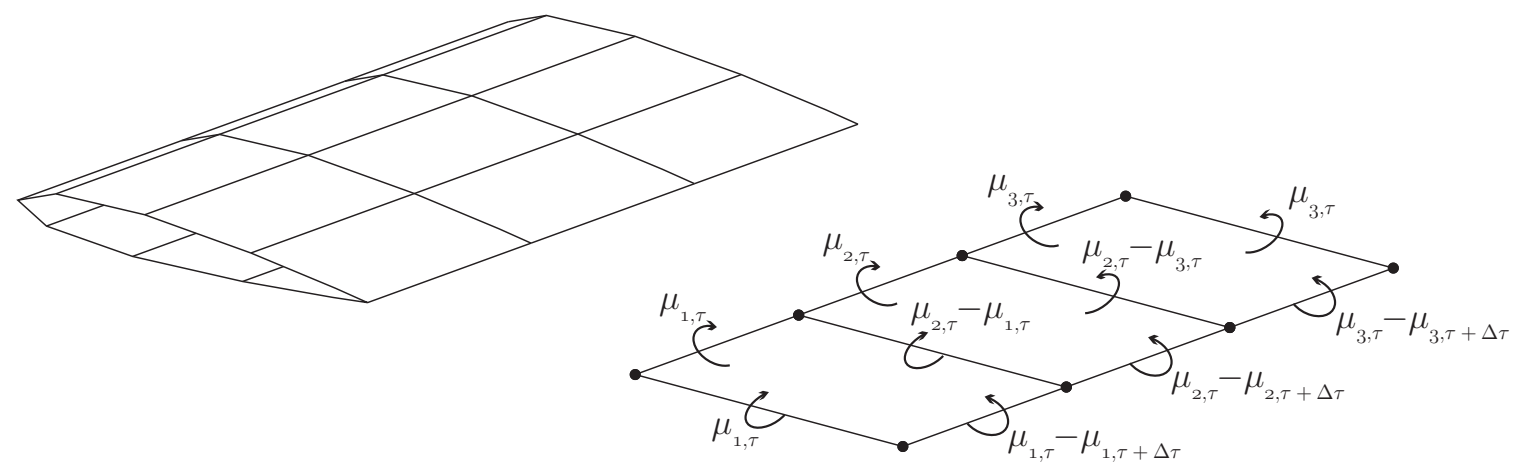

Figure A.2: Convected near-wake after conversion to vortex filament representation

The vortex filaments associated with three of the four edges of the each panel are converted to a vortex particle, as illustrated in Fig. A.3, A.4, and A.5. It is noted that any vortex filament which is shared between two adjacent near-wake panels will be treated as two coincident vortex filaments with equal strength.

Integration over each vortex filament which contributes to the creation of a vortex particle must conserve the zero and first moments of vorticity [48]. To satisfy this 


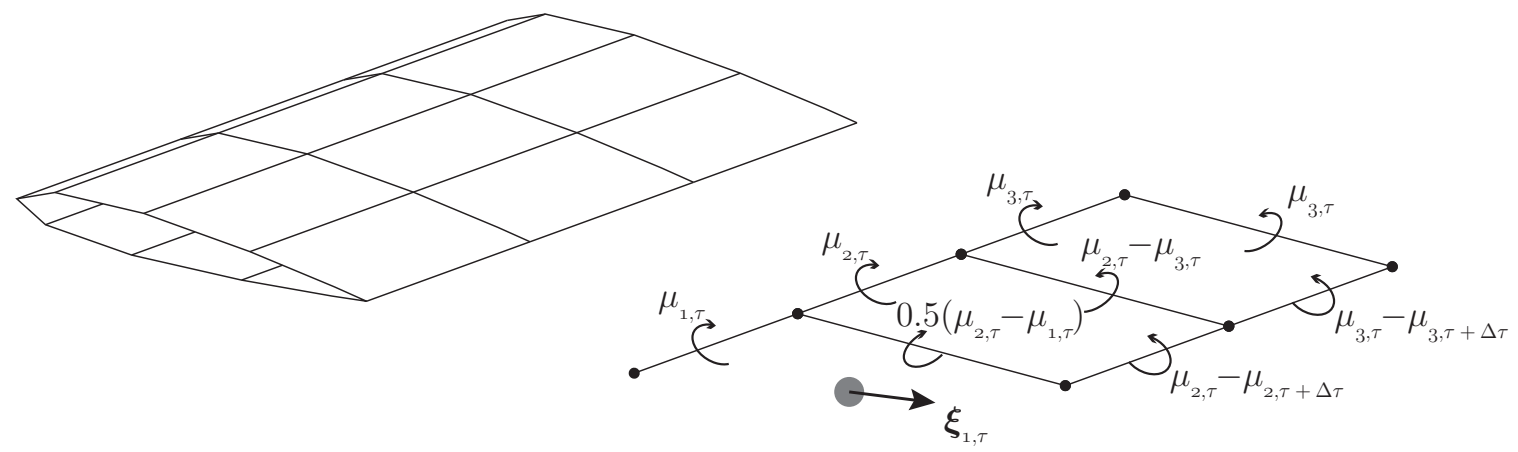

Figure A.3: Convected near-wake after conversion to vortex filament representation and one panel converted to a vortex particle

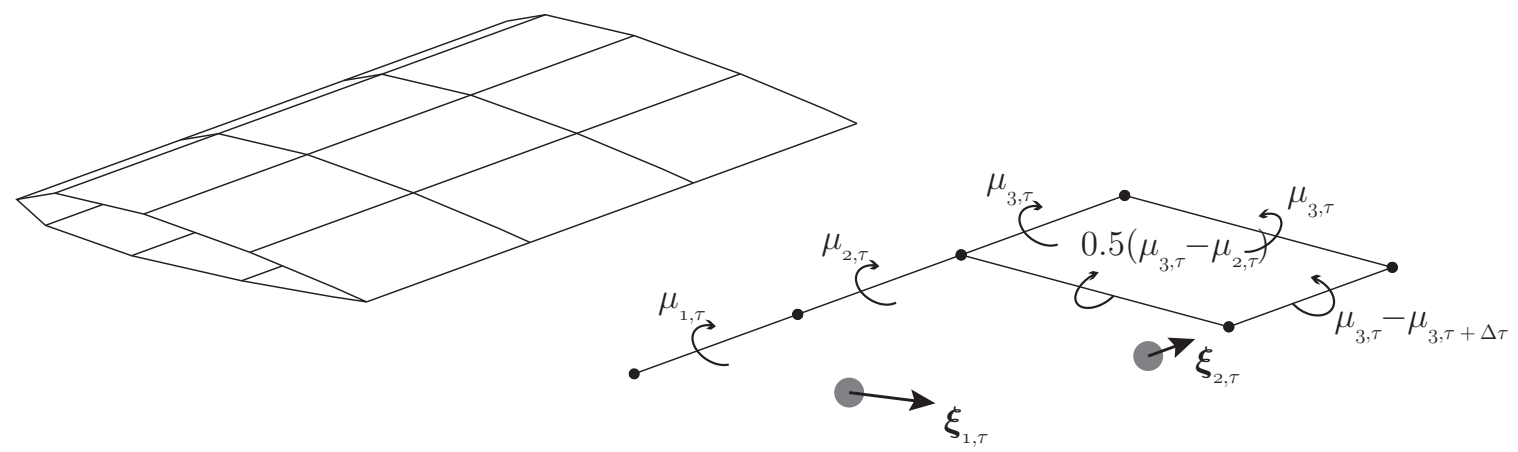

Figure A.4: Convected near-wake after conversion to vortex filament representation and two panels converted to vortex particles

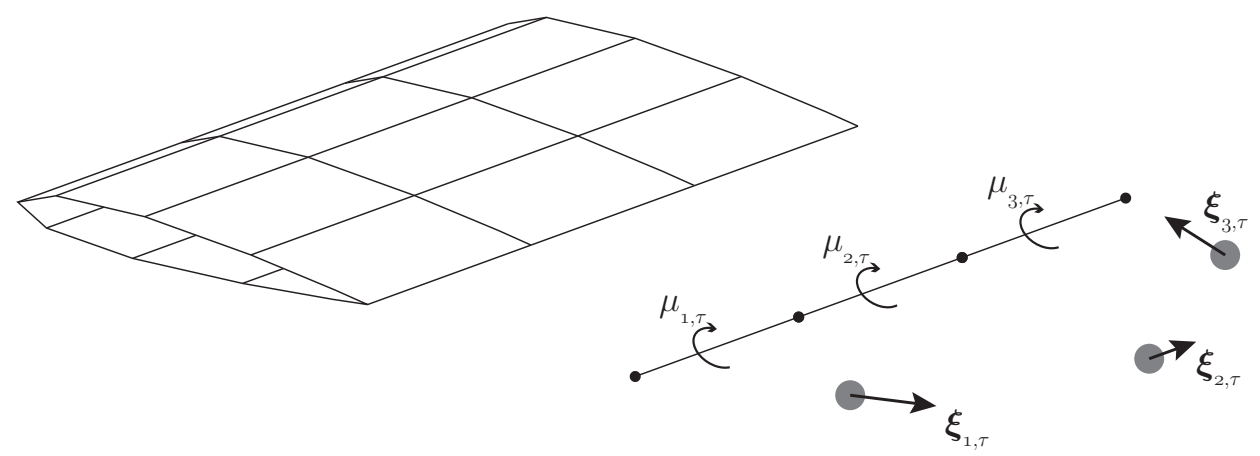

Figure A.5: Convected near-wake after conversion to vortex filament representation and three panels converted to vortex particles

condition, every contributing vortex filament $i$ with constant vorticity distribution $\gamma_{i}$ and length vector $\boldsymbol{\ell}_{i}$ is first considered as a point vortex located at the mid-point of 
the filament $\mathbf{r}_{i}$ with a vorticity of $\gamma_{i} \ell_{i}$. The two point vortices from the sides of the panel (associated with two of the vortex filaments in the stream-wise direction) are first combined into a single point vortex with vorticity $\boldsymbol{\Gamma}$ and position $\mathbf{q}$ where

$$
\begin{gathered}
\boldsymbol{\Gamma}=\gamma_{1} \ell_{1}+\gamma_{3} \ell_{3}, \\
\mathbf{q}=\frac{\gamma_{1} \ell_{1}}{\gamma_{1} \ell_{1}+\gamma_{3} \ell_{3}} \mathbf{r}_{1}+\frac{\gamma_{3} \ell_{3}}{\gamma_{1} \ell_{1}+\gamma_{3} \ell_{3}} \mathbf{r}_{3} .
\end{gathered}
$$

Next, this newly-formed point vortex is combined with the remaining point vortex (created from the vortex filament in the span-wise direction) to obtain a single point vortex with vorticity $\boldsymbol{\xi}$ and position $\mathbf{Z}$ using

$$
\begin{gathered}
\boldsymbol{\xi}=\gamma_{2} \ell_{2}+\boldsymbol{\Gamma}_{1}, \\
\mathbf{Z}=\frac{\gamma_{2} \ell_{2}}{\gamma_{2} \ell_{2}+\Gamma_{1}} \mathbf{r}_{2}+\frac{\Gamma_{1}}{\gamma_{2} \ell_{2}+\Gamma_{1}} \mathbf{q}_{1} .
\end{gathered}
$$

The resulting point vortex is the vortex particle, which has an intensity of $\boldsymbol{\xi}$ and is located at $\mathbf{Z}$.

The fourth edge of each convected wake panel which was not included in the integration is treated as a vortex filament. These remaining vortex filaments and the vortex particles then become part of the far-wake for the new time step, as shown in Fig. A.6. When the next row of near-wake panels is convected and converted into vortex particles, the previous vortex filament strengths are included in the TE of the convected wake panels. 


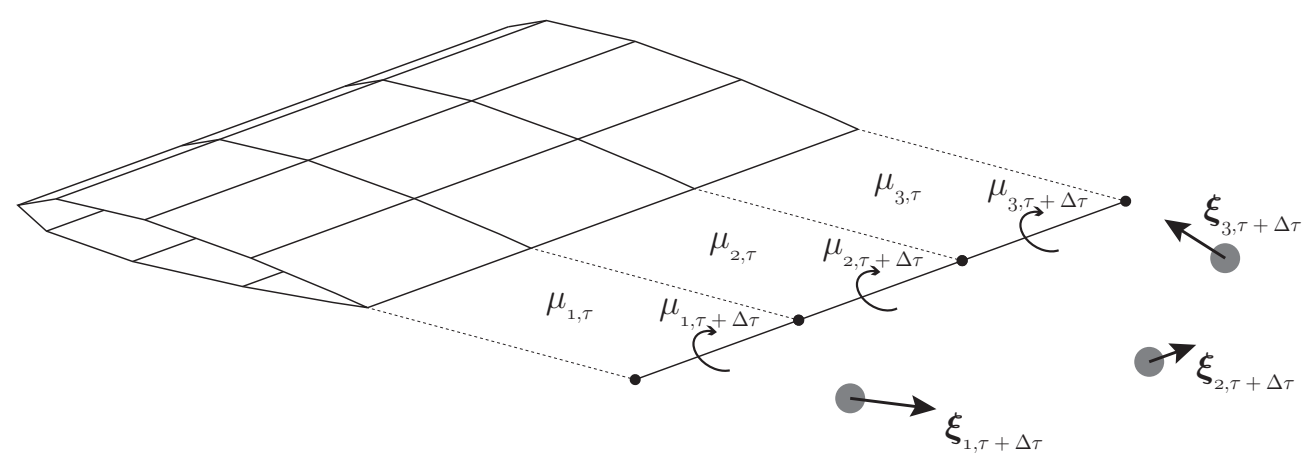

Figure A.6: The new near-wake doublet panels and far-wake vortex filaments and vortex particles 


\section{Appendix B}

\section{Investigating Aeroacoustic Formulations}

\section{B.1 Derivation of Formulation 1C for non- penetrable surfaces}

For a moving solid body immersed in a fluid without mean flow, the FW-H acoustic analogy considers an acoustic monopole and dipole source distributions over the body surface and a quadrupole source distribution in the volume around the body. The FW-H equation was originally proposed in [26] and has been expressed in [27] as

$$
\square^{2} p^{\prime}(\mathbf{x}, t)=\frac{\partial}{\partial t}\left[\rho_{0} v_{n} \delta(f)\right]-\frac{\partial}{\partial x_{i}}\left[p \hat{n}_{i} \delta(f)\right]+\frac{\partial^{2}}{\partial x_{i} \partial x_{j}}\left[H(f) T_{i j}\right]
$$

where $\square^{2}$ is the wave or D'Alembertian operator in three-dimensional space; $p^{\prime}$ is the acoustic or perturbation pressure for an observer at position $\mathbf{x}$ at time $t$; $v_{n}$ is the component of the velocity normal to the body; $p$ is the local gauge pressure on the body's surface; $f \equiv f(\mathbf{y})$ is the equation of the body surface such that $f<0$ inside the surface, $f>0$ outside the surface, and $f=0$ on the surface; $\hat{\mathbf{n}}=\boldsymbol{\nabla} f$ is the unit

outward normal of the surface; and, $H(f)$ and $\delta(f)$ are the Heaviside and Dirac delta functions, respectively. 
The first term on the right-hand side of Eq. (B.1) is the thickness noise contribution to acoustic pressure, the second term is the loading noise contribution, and the third term is the quadrupole noise contribution.

The surface defined by $f$ may also be considered penetrable, such as a data (nonphysical) surface that encapsulates the solid surface and all volume quadrupoles producing non-negligible noise. As in [27] and [33], the FW-H equation for this penetrable data surface becomes

$$
\square^{2} p^{\prime}(\mathbf{x}, t)=\frac{\partial}{\partial t}\left[Q_{n} \delta(f)\right]-\frac{\partial}{\partial x_{i}}\left[L_{n i} \delta(f)\right]+\frac{\partial^{2}}{\partial x_{i} \partial x_{j}}\left[H(f) T_{i j}\right]
$$

where

$$
\begin{gathered}
Q_{n}=\rho\left(u_{n}^{\prime}-v_{n}\right)+\rho_{0}\left(v_{n}\right), \\
L_{n i}=\rho u_{i}^{\prime}\left(u_{n}^{\prime}-v_{n}\right)+P_{i j} \hat{n}_{j}, \\
P_{i j}=p^{\prime} \delta_{i j}-\sigma_{i j},
\end{gathered}
$$

and $T_{i j}$ is Lighthill's stress tensor. For scenarios in which the quadrupole source is neglected or will not be directly solved, the third term on the right-hand side of Eq. (B.2) may be neglected.

For an impenetrable data surface such as a solid body, $\left(u_{n}^{\prime}-v_{n}\right)=0$ at all times, thereby removing the first terms from the right-hand sides of Eq. (B.3) and (B.4). The viscous shear forces acting on the data surface, $\sigma_{i j}$, may be neglected as in [27], leaving

$$
\begin{gathered}
Q_{n}=\rho_{0} v_{n}, \\
L_{n i}=p^{\prime} \delta_{i j} \hat{n}_{j}=p^{\prime} \hat{n}_{i} .
\end{gathered}
$$


These terms are related to the terms $Q_{i}$ and $L_{i j}$ in the convective FW-H equation presented in Najafi-Yazdi et al. [33] by

$$
\begin{gathered}
Q_{n}=Q_{i} \hat{n}_{i}, \\
L_{n i}=L_{i j} \hat{n}_{i},
\end{gathered}
$$

where

$$
\begin{gathered}
Q_{i}=\rho\left(u_{i}^{\prime}+U_{\infty i}-v_{i}\right)+\rho_{0}\left(v_{i}-U_{\infty i}\right), \\
L_{i j}=\rho u_{i}^{\prime}\left(u_{j}^{\prime}+U_{\infty j}-v_{j}\right)+P_{i j} .
\end{gathered}
$$

The non-penetration condition for the convective formulation requires

$$
\left(u_{i}^{\prime}+U_{\infty i}-v_{i}\right) \hat{n}_{i}=0
$$

causing the normal components of the first terms in the expressions for $Q_{i}$ and $L_{i j}$ to become zero, and therefore equal to $Q_{n}$ and $L_{n i}$ in Eq. (B.8) and (B.9), respectively.

Using these relationships, $Q_{n}$ and $L_{n i}$ may be substituted into the FW-H equation as

$$
p^{\prime}(\mathbf{x}, t)=\frac{\partial}{\partial t} \int_{f=0}\left[\frac{Q_{n}}{4 \pi|\mathbf{x}-\mathbf{y}|}\right]_{\mathrm{ret}} \mathrm{d} S-\frac{\partial}{\partial x_{i}} \int_{f=0}\left[\frac{L_{n i}}{4 \pi|\mathbf{x}-\mathbf{y}|}\right]_{\mathrm{ret}} \mathrm{d} S,
$$

where the subscript ret means that the contained expression is to be calculated at the emission (or retarded) time and position, $\mathbf{y}$ is the position of the aeroacoustic source, and $\mathbf{x}$ is the position of the observer.

Using a similar procedure and notation as [33] yields the following general expressions for loading and thickness acoustic pressures for a subsonic mean flow $\mathbf{U}_{\infty}$ along 
the positive $x_{1}$-direction:

$$
\begin{gathered}
4 \pi p_{T}^{\prime}=\left[\frac{\partial}{\partial t}+U_{\infty} \frac{\partial}{\partial x_{1}}\right] \int_{-\infty}^{t} \int_{\mathbb{R}^{3}} Q_{n} \delta(f) \frac{\delta(g)}{R^{*}} d^{3} \mathbf{y} \mathrm{d} \tau, \\
4 \pi p_{L}^{\prime}=-\frac{\partial}{\partial x_{i}} \int_{-\infty}^{t} \int_{\mathbb{R}^{3}} L_{n i} \delta(f) \frac{\delta(g)}{R^{*}} \mathrm{~d}^{3} \mathbf{y} \mathrm{d} \tau,
\end{gathered}
$$

where $g=\tau-t+R / c_{0}$.

Continuing with the procedure presented in [33], the thickness noise contribution to sound pressure becomes

$$
\begin{aligned}
4 \pi p_{T}^{\prime}= & \int_{f=0}\left[\frac{\dot{Q}_{n}}{R^{*}\left(1-M_{R}\right)^{2}}\right]_{\mathrm{ret}} \mathrm{d} \boldsymbol{\eta}+\int_{f=0}\left[\frac{-\partial R^{*}}{\partial \tau} \frac{Q_{n}}{R^{* 2}\left(1-M_{R}\right)^{2}}\right]_{\mathrm{ret}} \mathrm{d} \boldsymbol{\eta} \\
& +\int_{f=0}\left[\frac{Q_{n}}{R^{*}\left(1-M_{R}\right)^{3}} \frac{\partial M_{R}}{\partial \tau}\right]_{\mathrm{ret}} \mathrm{d} \boldsymbol{\eta} \\
& -M_{\infty} \int_{f=0}\left[\frac{\dot{\tilde{R}}_{1} Q_{n}+\tilde{R}_{1} \dot{Q}_{n}}{R^{*}\left(1-M_{R}\right)^{2}}\right]_{\mathrm{ret}} \mathrm{d} \boldsymbol{\eta} \\
& +M_{\infty} \int_{f=0}\left[\frac{\partial R^{*}}{\partial \tau} \frac{\tilde{R}_{1} Q_{n}}{R^{* 2}\left(1-M_{R}\right)^{2}}\right]_{\mathrm{ret}} \mathrm{d} \boldsymbol{\eta}-M_{\infty} \int_{f=0}\left[\frac{\partial M_{R}}{\partial \tau} \frac{\tilde{R}_{1} Q_{n}}{R^{*}\left(1-M_{R}\right)^{3}}\right]_{\mathrm{ret}} \mathrm{d} \boldsymbol{\eta} \\
& -U_{\infty} \int_{f=0}\left[\frac{\tilde{R}_{1}^{*} Q_{n}}{R^{* 2}\left(1-M_{R}\right)}\right]_{\mathrm{ret}} \mathrm{d} \boldsymbol{\eta},
\end{aligned}
$$

and the loading noise contribution becomes

$$
\begin{aligned}
& 4 \pi p_{L}^{\prime}=\frac{1}{c_{0}} \int_{f=0}\left[\frac{\dot{\dot{L}_{n i}} \tilde{R}_{i}+L_{n i} \dot{\tilde{R}}_{i}}{R^{*}\left(1-M_{R}\right)^{2}}\right]_{\mathrm{ret}} \mathrm{d} \boldsymbol{\eta} \\
& -\frac{1}{c_{0}} \int_{f=0}\left[\frac{\partial R^{*}}{\partial \tau} \frac{L_{n i} \tilde{R}_{i}}{R^{* 2}\left(1-M_{R}\right)^{2}}\right]_{\mathrm{ret}} \mathrm{d} \boldsymbol{\eta} \\
& +\frac{1}{c_{0}} \int_{f=0}\left[\frac{\partial M_{R}}{\partial \tau} \frac{L_{n i} \tilde{R}_{i}}{R^{*}\left(1-M_{R}\right)^{3}}\right]_{\text {ret }} \mathrm{d} \boldsymbol{\eta} \\
& +\int_{f=0}\left[\frac{L_{n i} \tilde{R}_{i}^{*}}{R^{* 2}\left(1-M_{R}\right)}\right]_{\mathrm{ret}} \mathrm{d} \boldsymbol{\eta},
\end{aligned}
$$


where dots above the variables represent temporal derivatives with respect to the source time $\tau$ and may be calculated numerically or analytically, and the vector $\boldsymbol{\eta}$ defines any point on the surface in the surface's local coordinate system.

When the data surface is attached to a rigid body rotating with an angular velocity of $\Omega$, the analytic solution for the derivative of the unit normal vector on the surface is

$$
\dot{\hat{\mathbf{n}}}=\boldsymbol{\Omega} \times \hat{\mathbf{n}}=\epsilon_{i j k} \Omega_{j} \hat{n}_{k},
$$

where $\epsilon_{i j k}$ is the Levi-Civita symbol. The source temporal derivatives of $Q_{n}$ and $L_{n i}$ may be calculated using

$$
\begin{gathered}
\dot{Q}_{n}=\frac{\partial}{\partial \tau}\left[\rho_{0}\left(v_{i}-U_{\infty i}\right)\right] \hat{n}_{i}+\rho_{0}\left(v_{i}-U_{\infty i}\right) \epsilon_{i j k} \Omega_{j} \hat{n}_{k}, \\
\dot{L}_{n i}=\frac{\partial p^{\prime}}{\partial \tau} \hat{n}_{i}+p^{\prime} \epsilon_{i j k} \Omega_{j} \hat{n}_{k} .
\end{gathered}
$$

The equations for thickness and loading noise contributions may be modified to suit a panel method implementation, where the data surface is discretized into $N$ flat panels which are considered to have constant values of perturbation pressure $p^{\prime}$ and surface velocity $\mathbf{v}$ over the panel area $S$ which has a unit normal vector $\hat{\mathbf{n}}$ and centroid at position $\mathbf{y}$. Concentrating the surface noise contributions of each panel to originate from their respective centroids allows for the integrations to become summations of the panels and yields the convective, non-penetrable form of the thickness and loading 
noise contributions:

$$
\begin{aligned}
4 \pi p_{T}^{\prime}\left(\mathbf{x}, t_{\text {adv }}\right)=\sum_{k=1}^{N} & {\left[\left(\frac{\dot{Q}_{n}}{R^{*}\left(1-M_{R}\right)^{2}}-\frac{\partial R^{*}}{\partial \tau} \frac{Q_{n}}{R^{* 2}\left(1-M_{R}\right)^{2}}+\frac{Q_{n}}{R^{*}\left(1-M_{R}\right)^{3}} \frac{\partial M_{R}}{\partial \tau}\right)\right.} \\
& -M_{\infty}\left(\frac{\dot{\tilde{R}}_{1} Q_{n}+\tilde{R}_{1} \dot{Q}_{n}}{R^{*}\left(1-M_{R}\right)^{2}}-\frac{\partial R^{*}}{\partial \tau} \frac{\tilde{R}_{1} Q_{n}}{R^{* 2}\left(1-M_{R}\right)^{2}}+\frac{\partial M_{R}}{\partial \tau} \frac{\tilde{R}_{1} Q_{n}}{R^{*}\left(1-M_{R}\right)^{3}}\right) \\
& \left.-U_{\infty}\left(\frac{\tilde{R}_{1}^{*} Q_{n}}{R^{* 2}\left(1-M_{R}\right)}\right)\right]_{k} S_{k},
\end{aligned}
$$

and

$$
\begin{aligned}
4 \pi p_{L}^{\prime}\left(\mathbf{x}, t_{\text {adv }}\right)=\sum_{k=1}^{N} & {\left[\frac{1}{c_{0}}\left(\frac{\dot{L}_{n_{i}} \tilde{R}_{i}+L_{n i} \dot{\tilde{R}}_{i}}{R^{*}\left(1-M_{R}\right)^{2}}-\frac{\partial R^{*}}{\partial \tau} \frac{L_{n i} \tilde{R}_{i}}{R^{* 2}\left(1-M_{R}\right)^{2}}+\frac{\partial M_{R}}{\partial \tau} \frac{L_{n i} \tilde{R}_{i}}{R^{*}\left(1-M_{R}\right)^{3}}\right)\right.} \\
& \left.+\frac{L_{n i} \tilde{R}_{i}^{*}}{R^{* 2}\left(1-M_{R}\right)}\right]_{k} S_{k} .
\end{aligned}
$$

Here, the terms on the right-hand side of Eq. B.21 and B.22 are evaluated at the emission source time $\tau_{e}$, and the observer time is calculated using the acoustic radiation distance $R$ at emission source time as

$$
t_{\mathrm{adv}}=\tau_{e}+\frac{R}{c_{0}} .
$$

